\title{
A Survey on Mobile Social Signal Processing
}

NIKLAS PALAGHIAS, University of Surrey

SEYED AMIR HOSEINITABATABAEI, University of Surrey

MICHELE NATI, University of Surrey

ALEXANDER GLUHAK, Intel Labs Europe

KLAUS MOESSNER, University of Surrey

Understanding human behaviour in an automatic but non-intrusive manner is an important area for various applications. This requires the collaboration of information technology with human sciences to transfer existing knowledge of human behaviour into self-acting tools. These tools will reduce human error that is introduced by current obtrusive methods such as questionnaires. To achieve unobtrusiveness, we focus on exploiting the pervasive and ubiquitous character of mobile devices.

In this article, a survey of existing techniques for extracting social behaviour through mobile devices is provided. Initially we expose the terminology used in the area and introduce a concrete architecture for social signal processing applications on mobile phones, constituted by sensing, social interaction detection, behavioural cues extraction, social signal inference and social behaviour understanding. Furthermore, we present state-of-the-art techniques applied to each stage of the process. Finally, potential applications are shown while arguing about the main challenges of the area.

Categories and Subject Descriptors: General and reference [Document Types]: Surveys and Overviews; Human-centered computing [Collaborative and social computing, Ubiquitous and mobile computing]

General Terms: Design, Theory, Human Factors, Performance

Additional Key Words and Phrases: Social Signal Processing, mobile phones, social behaviour

ACM Reference Format:

Niklas Palaghias, Seyed Amir Hoseinitabatabaei, Michele Nati, Alexander Gluhak and Klaus Moessner, 2014. A Survey on Mobile Social Signal Processing. ACM V, N, Article A (January YYYY), 35 pages.

DOI : http://dx.doi.org/10.1145/0000000.0000000

\section{INTRODUCTION}

Human behaviour understanding has received a great deal of interest since the beginning of the previous century. People initially conducted research on the way animals behave when they are surrounded by creatures of the same species. Acquiring basic underlying knowledge of animal relations led to extending this information to humans in order to understand social behaviour, social relations etc. Initial experiments were conducted by empirically observing people and retrieving feedback from them. These methods gave rise to well-established psychological approaches for understanding human behaviour, such as surveys, questionnaires, camera recordings and human observers. Nevertheless, these methods introduce several limitations including various sources of error. Completing surveys and questionnaires induces partiality, unconcern etc. [Groves 2004], human error [Reason 1990], and additional restrictions in scalability of the experiments. Accumulating these research problems leads to a common challenge, the lack of automation in an unobtrusive manner.

An area that has focussed on detecting social behaviour automatically and has received a great amount of attention is Social Signal Processing (SSP). The main target of the field is to model, analyse and synthesise human behaviour with limited user intervention. To achieve these targets, researchers presented three key terms which constitute different levels of abstraction in the process of educing social behaviour [Brunet et al. 2012] [Vinciarelli et al. 2012a] [Poggi et al. 2012]. Behavioural cues include various characteristics of human behaviour that are extracted from a modality such as prosody of the voice and interlocutors spatial arrangement. The combination of these behavioural characteristics indicate a person's current sentiment, understanding, attention, interest etc. which are social signals. Pentland [Pentland 2008] described social signals as non-verbal communication signals emitted when people are socially interacting. Merging these social signals in a longer temporal term leads to a person's social behaviour. In recent literature the terms have been used in other areas such as social networks [Iamnitchi et al. 2012] to indicate every social related Internet activity of a user. However, we do not consider

This work is supported by European Commission through the FP7-SOCIOTAL-609112 EU Projects and also by the joint EU and Ministry of Internal Affairs and Communication (MIC, Japan), Research and Innovation action iKaaS, under EU Grant No. 643262.

Author's addresses: N. Palaghias, S. A. Hoseinitabatabaei, M. Nati and K. Moessner, Institute for Communication Systems, University of Surrey; A. Gluhak, Intel Labs Europe, London.

Permission to make digital or hard copies of part or all of this work for personal or classroom use is granted without fee provided that copies are not made or distributed for profit or commercial advantage and that copies show this notice on the first page or initial screen of a display along with the full citation. Copyrights for components of this work owned by others than ACM must be honored. Abstracting with credit is permitted. To copy otherwise, to republish, to post on servers, to redistribute to lists, or to use any component of this work in other works requires prior specific permission and/or a fee. Permissions may be requested from Publications Dept., ACM, Inc., 2 Penn Plaza, Suite 701, New York, NY 10121-0701 USA, fax +1 (212) 869-0481, or permissions@acm.org.

(c) YYYY ACM 0000-0000/YYYY/01-ARTA $\$ 15.00$

DOI : http://dx.doi.org/10.1145/0000000.0000000

https://mc.manuscriptcentrah@om/csurv, No. N, Article A, Publication date: January YYYY. 
this aspect in behaviour inference. Social networks may function as an enhancement of SSP to provide additional information regarding the context but to our interpretation the two areas are distinct.

In [Vinciarelli et al. 2009] a generic procedure was proposed to detect social behaviour:

(1) Data capture.

(2) Person detection.

(3) Extraction of audio and visual behavioural cues, and their mapping to social signals.

(4) Incorporate context to detect social behaviour from social signals.

This procedure is focussed on detecting the social behaviour of people through audio and visual data, from an external observer's point of view. In order to achieve this observation, microphones and cameras are required to be deployed on the scene to monitor people. The major disadvantages of this approach are a) the system's intrusiveness as people feel monitored by seeing deployed obtrusive hardware such as microphones and cameras used primarily for surveillance in contrast to the utilisation of pervasive devices such as smartphones, b) limited mobility of the system, where in case of the requirement for conducting an experiment in a different area there is a need for re-deploying and re-configuring the system to the specific environment, c) the confinement in scalability because the equipment is deployed at a certain environment and cannot follow the user's mobility, d) in audio and visual data there is a need to perform person detection, while mining social interactions during which social signals are emitted, is neglected, and finally e) establishing ground truth in audio and visual data requires labelling that is a time-consuming process and may induce human error.

\subsection{A mobile and opportunistic point of view}

The purpose of this article is to review state-of-the-art techniques for extracting social behaviour through mobile phones and also to introduce a discussion on the remaining challenges, existing gaps and potential extensions of existing solutions of the area. Understanding social behaviour in an automatic, non-intrusive, mobile, but also scalable manner constitutes a significant challenge with several potential applications. To address this challenge, close collaboration is required from the fields that accord two of the most important components of the field, information technology and psychology. This collaboration will support the development of opportunistic non-intrusive self-acting tools for extracting human behaviour. These tools will expunge several sources of error introduced by current obtrusive and user engaging methods that incorporate human factor in the sensing process. In parallel, SSP focussed on providing concrete solutions regarding modelling, analysis and synthesis of social behaviour. However, as mentioned some major gaps have been identified.

In order to fill these gaps we determined the following objectives, which will drive research on extracting personalised social behaviour a step further.

— Utilising non-intrusive approaches.

- Capturing cues from user's perspective, to produce personalised data.

- Leverage multiple modalities, to extract more robust and reliable behavioural information.

- Continuous sensing and inference process, without mobility and scalability restrictions.

- Elimination of external hardware requirement.

Smartphones have become a core feature of our daily lives. In recent years, popularity and computational power of mobile phones have led to a new era where they are substituting computers and other means of communication such as old feature phones, fixed line phones etc. Moreover, to facilitate a more rich user experience, mobile phone manufacturers have integrated various sensors such as an accelerometer, gyroscope, GPS, digital compass, microphone, camera etc. Furthermore, on-line application stores have given the opportunity to third party developers to implement their own applications utilising available integrated sensors seamlessly. Combining embedded sensors and application stores will introduce radical changes in fields such as healthcare, environment monitoring and human behaviour recognition by allowing easy, non-intrusive and wide deployment of mobile applications.

Given the pervasive and ubiquitous character of mobile devices and considering the built in sensing features, we consider smartphones as ideal devices for extracting social behaviour among people. To support this claim, we introduce Mobile Social Signal Processing (Mobile SSP) while proposing the main architecture of human behaviour inference for mobile applications. Further, each stage is analysed by providing state-of-the-art techniques capable of being executed on mobile devices. Also, potential application cases will help to familiarise the reader with areas that will benefit from the growth of Mobile SSP, followed by a discussion of research opportunities that may be leveraged for further contribution to the field.

In the remainder of this article a survey for Mobile SSP is provided. Section 2 describes the overall area of Mobile SSP while clarifying the core terms of the field. A brief description of existing sensing frameworks is shown in Section 3 and assists the reader in the selection criteria. State-of-the-art techniques utilised to detect social interactions among people on mobile phones are presented in Section 4. Behavioural cues extracted on smartphones whilst informing about their advantages and disadvantages are described in Section 5. Section 6 showcases methods of mining social signals and mapping them to social behaviours. Section 7 describes existing and potential applications of Mobile SSP. An overall discussion about methods presented for extracting social behaviour on mo- 




Fig. 1: Application architecture on Mobile Social Signal Processing.

bile phones is argued in Section 8. Finally, challenges of the area are outlined in Section 9 and the article concludes with Section 10.

\section{AN OVERVIEW OF MOBILE SOCIAL SIGNAL PROCESSING}

Mobile devices and in particular smartphones are ubiquitous. Multi-modal sensing capabilities combined with increased computational power and available tools for mobile application development led to the view that smartphones are ideal devices for filling the gap of lack of automation in social behaviour understanding. Users can easily install an app from on-line application stores without any geographical restrictions and the device will automatically become a human behaviour aware smartphone. To discriminate and categorise more easily the types of applications two classes have been defined: participatory are the social behaviour detection applications that require the user's participation in the sensing process and opportunistic where the user is not involved in the process [Lane et al. 2010]. In order to minimise the obtrusiveness of the system and secure the user's spontaneous behaviour, the main attention of the work is focussed on opportunistic social behaviour detection applications.

In the area of SSP, users are continuously monitored through surveillance hardware, however explicit cameras and microphones create the perception that the user is being monitored. Depending on the intrusiveness of the hardware utilised in the sensing process, peoples' spontaneous behaviour may be less affected, which may allow them to adapt faster. An inherit characteristic of the area of Mobile SSP is that users are being monitored but with pervasive devices such as smartphones. The main usage of smartphones relates to everyday routine processes such as phone calls, messaging, web browsing, gaming etc. As such, monitoring through these types of devices may affect less the spontaneity of users' social behaviour contrary to the utilisation of dedicated surveillance hardware. Also, as users install the social behaviour app, they give their permission for each type of sensed data, as opposed to the case of surveillance hardware where monitored users do not have any control over the sensed data. Nevertheless, independent of the sensing technology, the spontaneity of people feeling monitored may be affected. Taking this fact into account is crucial for the understanding of social behaviour [Pentland 2008].

Similar to [Vinciarelli et al. 2010] and based on the literature review we concluded that the following steps need to be taken for extracting social behaviour on mobile devices (See Fig. 1).

(1) Sensing.

(2) Social Interaction Detection.

(3) Extraction of Behavioural Cues.

(4) Understanding Social Behaviour by Inferring Social Signals.

Social behaviour inference on mobile devices is initiated by the Sensing process. During daily life, users emit behavioural cues and social signals, which are captured by sensors of the mobile device. These sensors may be integrated in the device or enclosed in external hardware that communicates with the mobile device. Each sensor detects a particular modality, then it converts the detected signal into a raw data signal. The result is processed into a desired format or is directly forwarded to the next stage of social behaviour inference. Researchers have developed various sensing frameworks to allow developers to collect data in an abstract and uniform way, while in some cases they also include an inference engine.

Pentland recognised the emission of social signals during a social interaction [Pentland 2007]. This signifies the importance of recognising social interactions before initiating the process of social behaviour understanding. After retrieving the appropriate data from mobile device's sensors, Social Interaction Detection may be performed as a preprocessing step of social behaviour inference. Understanding social interactions provides important contextual information that may be leveraged in the next steps of social behaviour inference. The knowledge of on-going social 


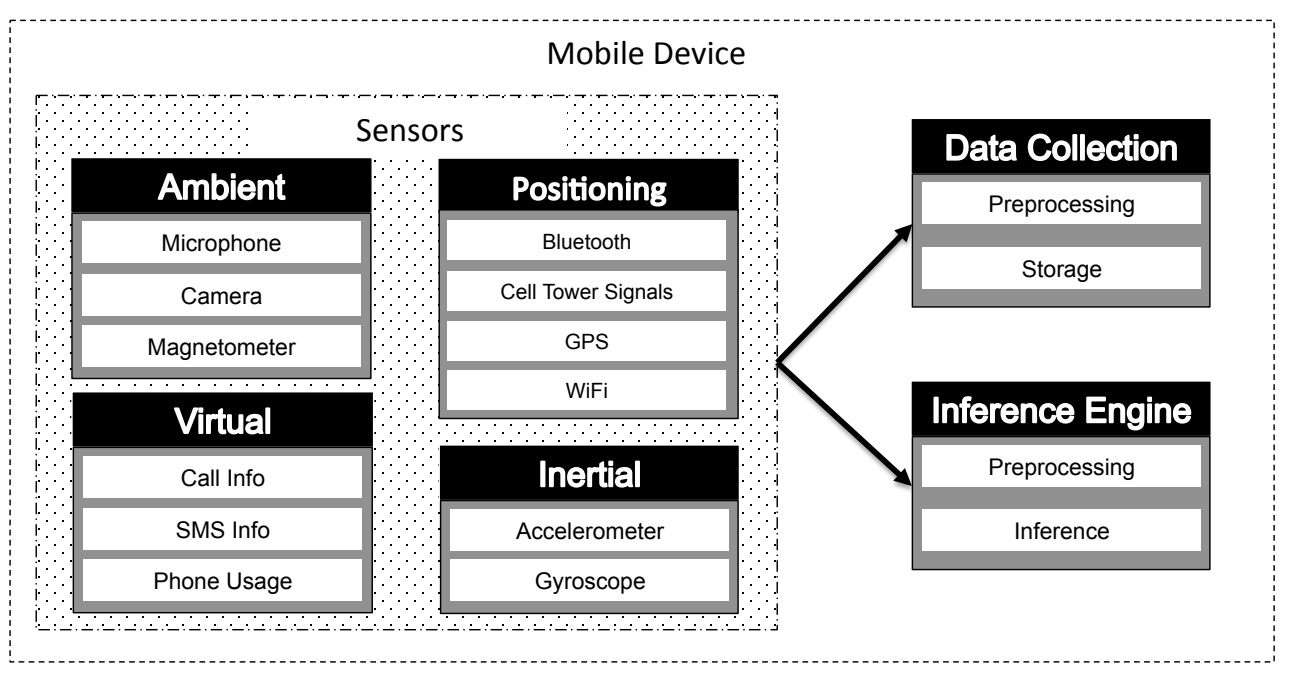

Fig. 2: Application architecture of existing Sensing Frameworks.

interactions may also be utilised for filtering data and allowing the development of adaptive sensing and inference techniques. In applications focusing on extracting behavioural information not related to the social aspect of a person, it is strongly encouraged to include this step as it provides important contextual information.

Following the identification of on-going social interactions is the extraction of behavioural cues. Different modalities may be leveraged for the extraction of a behavioural cue, depending on the grammar defined in psychology. Each selected sensed modality is forwarded to behavioural cues extraction. Existing literature has been classified into seven categories based on the types of cues each work extracts (See Fig. 1). The behavioural cues extraction is achieved through techniques such as decision models, statistical analysis etc.

The final stage of Mobile SSP is the transition from the understanding of social signals to social behaviour inference. Close collaboration with social sciences may provide the theoretical mapping among behavioural cues, social signals and social behaviours. Literature has been grouped based on the inferred social behaviour through mobile phones. The extracted behavioural cues are fed in decision making techniques to mine social signals and infer in long-term social behaviour.

To facilitate the reader's understanding of the field, we provide an outline of the main steps and requirements for an integrated and real-world-enabled Mobile SSP:

- Define the context of the Mobile SSP application.

— Select the modalities required to infer a particular social behaviour.

- Define the grammar of behavioural cues and social signals that will lead to social behaviour inference.

— Evaluate and verify the reliability of the approach in a real-world environment based on ground truth.

In addition to the above requirements, researchers need to consider the intrusiveness, security and privacy of the system. Researchers need to take into account the computational burden and energy consumption which may endanger user experience. These parameters do not constitute a prerequisite for the realisation of Mobile SSP but will facilitate user experience and privacy.

In the following sections, each of the pre-defined stages will be analysed and state-of-the-art research are outlined. The works described in the next sections are summarised in the Electronic Appendix, introducing the techniques developed in each stage of social behaviour inference.

\section{SENSING FRAMEWORKS}

Sensing is the first stage in extracting human behaviour on mobile devices. In this stage, selection of appropriate modalities is performed. These will later on be processed and analysed to reveal information about user's social behaviour. It constitutes the lowest level of the process, which collects raw data from sensors and other interfaces that can provide information relevant to the user (See Fig. 2). After retrieving information from sensors either the raw data are forwarded to the next stages or lightweight and simplistic processing may be performed to minimise the complexity and computational burden at the upcoming stages. As shown in Fig. 1, the next stages in social behaviour inference may be performed either on the device or at a backend server.

This section introduces and then compares existing sensing frameworks. Through this introduction, the reader should be able to understand the criteria based on which sensing framework should be selected for a desirable social behaviour application. An extensive analysis of existing sensing frameworks is outside the scope of this article and the reader is referred to [Lane et al. 2010] and [Hoseinitabatabaei et al. 2013]. 
Table I: Data collection frameworks for offline analysis

\begin{tabular}{|c|c|c|c|c|c|c|c|}
\hline Framework & \multicolumn{9}{|c|}{ Sensor Types } & $\begin{array}{c}\text { Energy } \\
\text { Efficient }\end{array}$ & Privacy & License \\
\cline { 2 - 8 } & Inertial & Ambient & Position & Virtual & $\checkmark$ & & \\
\hline MyExperience [Froehlich et al. 2007] & & $\checkmark$ & $\checkmark$ & $\checkmark$ & & & \\
SeeMon [Kang et al. 2008] & $\checkmark$ & $\checkmark$ & $\checkmark$ & $\checkmark$ & $\checkmark$ & & \\
Anonysense [Cornelius et al. 2008] & $\checkmark$ & $\checkmark$ & $\checkmark$ & $\checkmark$ & & $\checkmark$ & \\
OpenDataKit [Anokwa et al. 2009] & $\checkmark$ & $\checkmark$ & $\checkmark$ & $\checkmark$ & & & \\
PRISM [Das et al. 2010] & $\checkmark$ & $\checkmark$ & $\checkmark$ & $\checkmark$ & $\checkmark$ & & \\
LiveLab [Shepard et al. 2011] & $\checkmark$ & $\checkmark$ & $\checkmark$ & $\checkmark$ & & $\checkmark$ & \\
SystemSens [Falaki et al. 2011] & $\checkmark$ & $\checkmark$ & $\checkmark$ & $\checkmark$ & & & Custom \\
Funf [Funf 2011] & $\checkmark$ & $\checkmark$ & $\checkmark$ & $\checkmark$ & & & GNU GPL \\
Medusa [Ra et al. 2012] & $\checkmark$ & $\checkmark$ & $\checkmark$ & & & & Apache 2.0 \\
METIS [Rachuri et al. 2013] & $\checkmark$ & $\checkmark$ & $\checkmark$ & $\checkmark$ & $\checkmark$ & & \\
MSF [Cardone et al. 2013] & $\checkmark$ & $\checkmark$ & $\checkmark$ & $\checkmark$ & & & \\
\hline
\end{tabular}

\subsection{Data Collection}

This subsection focuses on sensing frameworks that perform only data collection and some minor pre-processing allowing the extraction of human behaviour off-line. An application is deployed on the device, which collects data from pre-configured sensors, and then either stores the information on device's internal storage or performs uploading to a backend server. The retrieved data are merged and forwarded to the next stage in order to extract behavioural cues. Table I summarises existing frameworks for data collection by presenting a) the type of sensor data retrieved, b) the incorporation of a mechanism for energy efficiency such as adaptive sensing, c) the embodiment of a privacy preserving approach and finally d) the software license through which the authors released a particular framework.

MyExperience [Froehlich et al. 2007] retrieves and logs contextual information from physical and virtual (e.g. phone usage patterns) sensors. SeeMon [Kang et al. 2008] investigates the context of the device and adapts the sensing process, by mining local sensors and installed applications in an energy efficient manner. To preserve privacy in crowd-sensing applications, [Cornelius et al. 2008] introduced Anonysense that permitted, through a centralised sensing coordination, the collection and storage of anonymous smartphone sensed data in a collaborative manner. OpenDataKit [Anokwa et al. 2009] is a set of tools for sensing and aggregating data from mobile phones. Furthermore, [Das et al. 2010] developed PRISM a platform for dynamic deployment of participatory and opportunistic sensing applications on mobile phones, while maintaining security through a sandboxed environment. Another approach that focuses on privacy-preservation but also on energy efficiency for long-range duration deployment is LiveLab [Shepard et al. 2011], which stores inertial, phone usage, positioning and network-based data. SystemSens [Falaki et al. 2011] is a data retrieval tool that enables researchers in capturing mobile data in large scale experiments. Funf [Funf 2011] is a mobile data collection platform developed by MIT Media Labs, procuring easily configurable sensing and data retrieval capabilities. Medusa [Ra et al. 2012] achieved crowd-sensing and simultaneous coordination of multiple mobile devices. METIS [Rachuri et al. 2013] is a distributed system that decides, based on the device status and user context, to perform on-device or infrastructure-oriented sensing. $M S F$ [Cardone et al. 2013] is a recent data collection framework that complies to multi-pipeline architecture and targets in providing an abstraction regarding the sensing process.

Discussion. Examining the data collection frameworks, MyExperience [Froehlich et al. 2007] constitutes an event triggered approach that is energy efficient and does not require any polling process to identify state changes but does not utilise data from inertial sensors. OpenDataKit [Anokwa et al. 2009], SystemSens [Falaki et al. 2011] and Funf [Funf 2011] are three distinct configurable open-source data collection tools that allow off-line merging of data from different sensors. However, they do not perform adaptive sensing based on the context to improve energy efficiency. This is critical for this type of applications. Medusa [Ra et al. 2012] allows a coordinator to retrieve a certain type of sensor-data from a specific device. Furthermore, METIS [Rachuri et al. 2013] is the first work that lightens a mobile device by selectively perform sensing through the infrastructure but simultaneously narrows the mobility and increases the intrusiveness of the system. MSF [Cardone et al. 2013] is focussing on easing the development of sensing applications. It requires the designer to implement the sensing functionality but handles tasks, power management and resource allocation. However, it does not constitute a ready to deploy solution and is suitable only for developers.

\subsection{Inference Engines}

As opposed to the previous subsection, this section includes frameworks that perform sensing and inference on the device or on a backend server. The sensor selection is predefined or configurable depending on the implementation. Data are retrieved from the sensors and forwarded to an inference pipeline. Based on the inference, one or more preprocessing stages could be performed. Then, the appropriate intelligence is applied to retrieve the requested knowledge. An optional post-processing phase, such as the consideration of historical inferences, outlier detection, smoothing etc., may be applied to remove results that deviate from normal. Table II presents the state-of-the-art 
Table II: Inference engine frameworks for on-line analysis

\begin{tabular}{|c|c|c|c|c|c|c|c|}
\hline Framework & \multicolumn{9}{|c|}{ Detection } & Energy & \multirow{2}{*}{ License } \\
\cline { 2 - 7 } & Activity & Voice & Location & Emotion & Sociability & Efficient & \\
\hline CenceMe [Miluzzo et al. 2008] & $\checkmark$ & $\checkmark$ & & & & & \\
BeTelGeuse [Kukkonen et al. 2009] & $\checkmark$ & & $\checkmark$ & & & & \\
Jigsaw [Lu et al. 2010] & $\checkmark$ & $\checkmark$ & $\checkmark$ & & & & \\
EmotionSense [Rachuri et al. 2010] & $\checkmark$ & $\checkmark$ & $\checkmark$ & $\checkmark$ & & $\checkmark$ & ISC \\
SociableSense [Rachuri et al. 2011] & $\checkmark$ & $\checkmark$ & $\checkmark$ & & $\checkmark$ & $\checkmark$ & ISC \\
AmbientDynamix [Carlson and Schrader & $\checkmark$ & $\checkmark$ & $\checkmark$ & & & & \\
2012] & & & & & & & \\
Auditeur [Nirjon et al. 2013] & & $\checkmark$ & & & & & \\
\hline
\end{tabular}

inference engines and identifies a) the type of information that is extracted by each framework, b) the development of an energy efficient approach and c) the software license of the framework.

CenceMe [Miluzzo et al. 2008] is a distributed platform that performs multi-modal sensing through mobile phones. A classification-based technique decides about inferring social context on the device or on a backend server. It also allows the user to publish the inference to social networks. BeTelGeuse [Kukkonen et al. 2009] was one of the first tools that had the native capability of sensing and inferring about user's context. Jigsaw [Lu et al. 2010] is a mobile platform that allows continuous data collection in an energy efficient way, through multiple pipelines (one for each modality) and adaptive sampling based on user behavioural patterns. EmotionSense [Rachuri et al. 2010] is a framework for inferring user emotion and incorporates an intelligent engine for adapting the sensing process. As an extension of it, SociableSense [Rachuri et al. 2011] measures sociability of people and introduces an adaptive inference mechanism (locally or distributed) based on reinforcement learning. AmbientDynamix [Carlson and Schrader 2012] is an equally important framework that allows the deployment of custom inference modules in a sandboxed environment. Also Auditeur [Nirjon et al. 2013] is a context recognition framework that is focussed only on the audio perspective, but provides a collection of inference mechanisms for the specific modality.

Discussion. Regarding state-of-the-art sensing frameworks that have a human behaviour inference, CenceMe [Miluzzo et al. 2008] performs preliminary detection of activity and conversation. However only an application that publishes user context to social networks is publicly available. BeTelGeuse [Kukkonen et al. 2009] focussed mainly on the sensing process, enabling the integration of external Bluetooth-connected sensors. It also allowed the incorporation of inference through plug-ins while initially providing location and activity classification components. Jigsaw [Lu et al. 2010] limits its sensing capabilities to the accelerometer, microphone and GPS but provides integrated classification techniques for activity and voice recognition. Through a multi-threaded approach they try to limit the computational burden on the device due to the classification process. EmotionSense [Rachuri et al. 2010] and SociableSense [Rachuri et al. 2011] are based on the same framework, providing a quantification method for the user's emotion and sociability whilst performing adaptive inference through learning techniques. It is available for developers but also for direct utilisation of the application for less technical people.

Furthermore, AmbientDynamix [Carlson and Schrader 2012] allows the user to select existing or concrete components, integrate them in a main skeleton application and perform the desirable social behaviour detection. If the component exists, it constitutes an easy and reliable solution while if the module requires development it can be contributed to the community for further reuse. All the processes of sensing configuration, data logging, resource management, concurrent procedures are handled in a seamless manner by the skeleton application, which reduces the developer's effort. If the targeting system is focussed on mining social behaviour information through audio data, Auditeur [Nirjon et al. 2013] constitutes a reasonable solution that provides the appropriate mechanisms to extract audio features but also allows the configuration of the desired classifier. In addition, it includes state-of-the-art techniques for contextual sound recognition.

\subsection{Framework Comparison}

A notable amount of works targeting sensing frameworks for mobile phones was briefly described in the two previous subsections. The literature was classified based on whether the framework enabled human behaviour inference or not.

Overall, the first step in the design of a social behaviour detection application is the decision about the sensing framework. Many researchers start by designing and developing the sensing process from scratch. However as shown, works on sensing frameworks have reached a certain maturity which allows component reuse. These frameworks provide off-the-shelf solutions for resource management, concurrency, data handling, energy efficiency and concrete structure of the application. This should be leveraged in order to reduce the development time cycle, human error and increase code reuse. Most of them are released with open licences, allowing clear understanding, editing but also contributing of the source code, from the research community. Selecting a data collection or inference engine framework is highly dependent on the targeting application and how sufficient the capabilities are of each framework with respect to the researchers' envisioned outcome. Thus, it should be noted that selecting a 
certain framework does not lead to a right or wrong decision but in a tool that will provide more or less enabling capabilities for developing a social behaviour detection application.

\section{SOCIAL INTERACTION DETECTION}

The next stage of retrieving data from sensing the context continues is recognising ongoing social interactions. People are assumed to interact socially, when they are in close interpersonal distance ${ }^{1}$, facing each other and participating in a conversation. Pentland definition of social signals [Pentland 2007], is that they are non-verbal communication signals that are conveyed when people are socially interacting. Thus, identifying possible social interactions accurately is an important stage of social behaviour understanding and requires tackling.

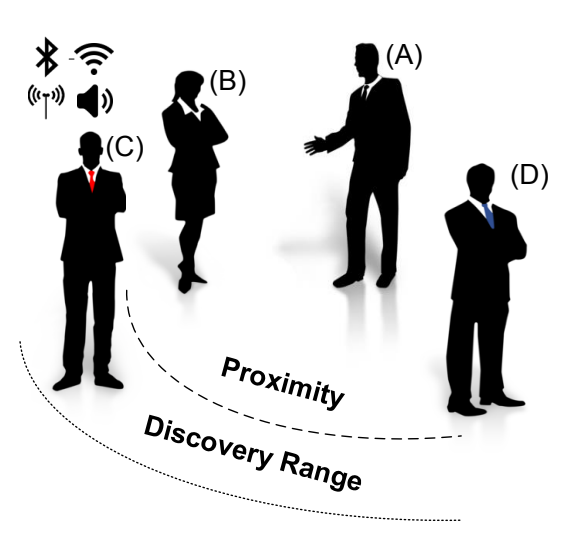

(a) Single Modality

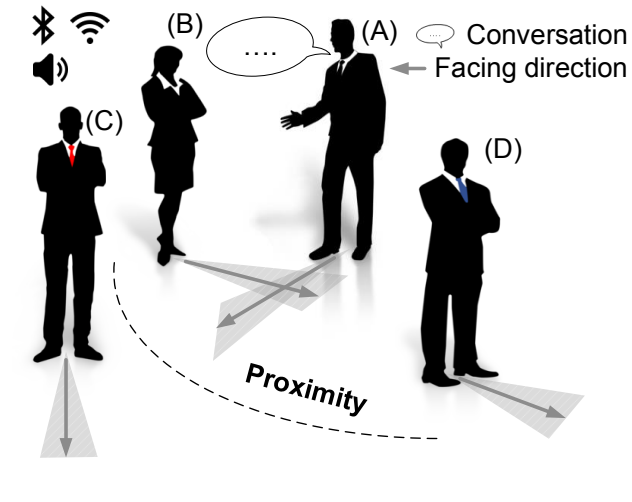

(b) Multiple modalities

Fig. 3: State-of-the-art social interaction detection approaches.

Researchers have developed several techniques to detect social interactions through smartphones. These techniques vary based on the level of accuracy and modalities included in the inference process. Among these approaches are single modality that include coarse or fine grain distance estimation through Bluetooth and WiFi interfaces, audio-based distance or relative position estimation. There is also multi-modality where different modalities are combined in the inference process to indicate if people are interacting (See Fig. 3).

\subsection{Single Modality}

The majority of commercial off-the-shelf (COTS) smartphones today comprise wireless communication interfaces such as Bluetooth and WiFi. Due to their wide availability researchers often utilised them in order to detect when people are in proximity. By acquiring information about people being in proximity, researchers made a strong assumption of interpreting the knowledge of proximity into the existence of social interaction. An implicit assumption is that every person is required to carry a smartphone. For the determination of proximity, several techniques have been proposed.

The most common approach is to perform discovery through one of the two interfaces, log the Bluetooth IDs (BTIDs) or WiFi Service Set Identifier (SSID) and classify all the detected nearby devices as social interactions. This method was applied in miscellaneous works to estimate when people are interacting with each other. Some examples of these works are Serendipity [Eagle and Pentland 2005], CenceMe [Miluzzo et al. 2008] and SoundSense [Lu et al. 2009]. [Antoniou et al. 2011] aggregated the discovered smartphones based on BTIDs with static nodes. The Bluetooth ranges were overlapping to improve social interaction detection and provide some information about coarse-grain localisation. The accuracy of this method is limited to the range of the communication mean i.e. for Bluetooth the minimum nominal range is around $10 \mathrm{~m}$ [Kotanen et al. 2003] and for WiFi the typical range is approximate to $35 \mathrm{~m}$ for indoor environment ${ }^{2}$. Thus, every device - person detected is classified as being in a social interaction. It should be noted that these works do not provide error analysis of this social interaction detection approach.

The previous method introduces a noticeable amount of error. For that reason researchers focussed on developing distance estimation techniques that would remove a percentage of error from the previous approach by limiting the

\footnotetext{
${ }^{1}$ The classification of the techniques between the sections of social interaction detection (Section 4) and the behavioural cue of interpersonal distance (Section 5.5.1) has been performed based on the context in which they are utilised in the literature. It should be noted that the social interaction detection techniques that are based only on proximity sensing may also be utilised for extracting the behavioural cue of interpersonal distance and vice versa.

${ }^{2}$ Bluetooth and WiFi ranges are highly dependent on the surrounding environment and chipsets characteristics of devices performing the discovery and the detected devices.
} 
communication mean range. So, for detecting social interactions [Osmani et al. 2014] developed a machine learning based technique to estimate if users were in proximity or not, by retrieving WiFi Received Signal Strength Indicator (RSSI). They trained a model based on maximum and mean value of a 20 -sample window of WiFi RSSI achieving a median error of $0.5 \mathrm{~m}$ for social interaction detection. In particular, they detected ongoing social interactions with $86 \%$ accuracy and true negatives with $84 \%$.

Another approach for detecting social interactions through Bluetooth RSSI-based distance estimation was presented in [Liu et al. 2013a]. They developed a probabilistic model for indoor and outdoor environments. It utilised some predefined empirically calculated thresholds to compute the probability of being in proximity to socially interact, with a claimed error rate of $4.3 \%$. In detail, they retrieved an RSSI measurement which was smoothed through exponential window moving average (EWMA) and a smoothing factor of 0.5. To compute the thresholds they retrieved Bluetooth RSSI measurement in different environments and distances. Bluetooth technology natively operates in a mode which allows the device to discover but also to be discoverable by other devices without any firmware modifications. A less complex approach was presented in [Hu et al. 2013], namely MAUC. This work focussed on detecting social interactions through Bluetooth RSSI thresholds, showing a detection rate over $90 \%$. As opposed to previous approaches, it incorporated an adaptive discovery interval scheme, dependent on user's activity movement.

Discussion. Scientists tackled the detection of social interactions on mobile phones with different granularity. The discovery of nearby devices (e.g. Serendipity [Eagle and Pentland 2005], CenceMe [Miluzzo et al. 2008] etc.) is easily implementable. However, it provides increased number of false positives due to inaccuracy in the interpersonal distance estimation, unawareness of spatial arrangement and conversation existence. As an example, Fig. 3a shows four people in vicinity, where only (A) and (B) are interacting, although all four are in discovery range, thus classified as socially interacting. The WiFi interface on COTS smartphones cannot operate simultaneously in discovering and discoverable mode as opposed to WiFi Direct. For that reason, the authors [Carreras et al. 2012] [Matic et al. 2012] were forced to modify the firmware of the devices to switch between the two modes. As an improvement, several works tried to estimate the interpersonal distance of users, to infer the existence of social interactions. For distance estimation based on RF (Bluetooth or WiFi) signals, RSSI has been leveraged in order to create empirical models, mainly due to simplicity in implementation. However, RSSI measurements received on mobile phones showcase great fluctuation which is affected by different environments, obstacles, human body absorption, reflections etc. Machine learning techniques constitute a notable effort to tackle the RSSI fluctuation [Osmani et al. 2014]. Threshold-based approaches [Liu et al. 2013a] [Hu et al. 2013] usually require adjustment of the algorithm's boundaries based on the device and the environment. Other techniques such as Time Difference of Arrival (TDoA), Angle of Arrival (AoA) and Time of Arrival (ToA) provide significant limitations such as firmware modification for device time synchronisation, lack of multiple antennas, the need for external hardware and therefore are not recommended for COTS mobile phones.

From simple discovery-based method, researchers have improved the social interaction detection through proximity. However, the assumption that when people are in proximity then are definitely interacting is strong. Hence, there is an imperative need to add other modalities in the inference process which will add new parameters such as spatial arrangement and conversation existence detection.

\subsection{Multi-modality}

To improve social interaction detection based on a single modality, researchers started to incorporate other modalities. These modalities enhanced social interaction detection by providing information about users' relative orientations i.e. if they are facing each other or not and about the conversation existence i.e. if the users are really having a conversation and they are not two people having a spatial formation suitable for social interaction (See Fig. 3b).

An important attempt to identify the relative spatial arrangement of the users combined with proximity detection was Virtual Compass [Banerjee et al. 2010]. This system utilises multiple RF interfaces such as Bluetooth and WiFi in order to estimate the interpersonal distance among users but also create a 2D localisation map based on users' relative distances. For distance estimation given RSSI measurements from both interfaces, they computed the average distance and the uncertainty based on the difference of $90^{t h}$ and $10^{t h}$ distance percentile. Then, the authors applied regression on these features for distance estimation and achieved an error margin of $1.41 \mathrm{~m}$. For relative map localisation they propose a quick technique to estimate the initial coordinates of each user then they apply an iterative method [Dabek et al. 2004] to refine the initially reckoned coordinates.

Matic [Matic et al. 2012] argued about a slightly different multi-modal approach that incorporated interpersonal distance estimation merged with relative orientation calculation and conversation detection. In this work, authors endeavoured to increase the accuracy of social interaction detection by taking into consideration users' facing directions. The knowledge of user's facing direction with respect to earth's coordinates allowed them to compute the relative orientation of each pair of users, in order to understand if they had the appropriate spatial arrangement to interact. They estimated the interpersonal distance of the users through an initial calibration phase which led to a proximity detection model. During that period, they collected WiFi RSSI measurements at 1m distance and then based on an indoor path loss model (PLM), they created an artificial dataset for distances $0.5 \mathrm{~m}, 1 \mathrm{~m} . . ., 5 \mathrm{~m}$. Based on this dataset they computed the mean and maximum value of a 20 -sample window and trained a Naïve Bayes 


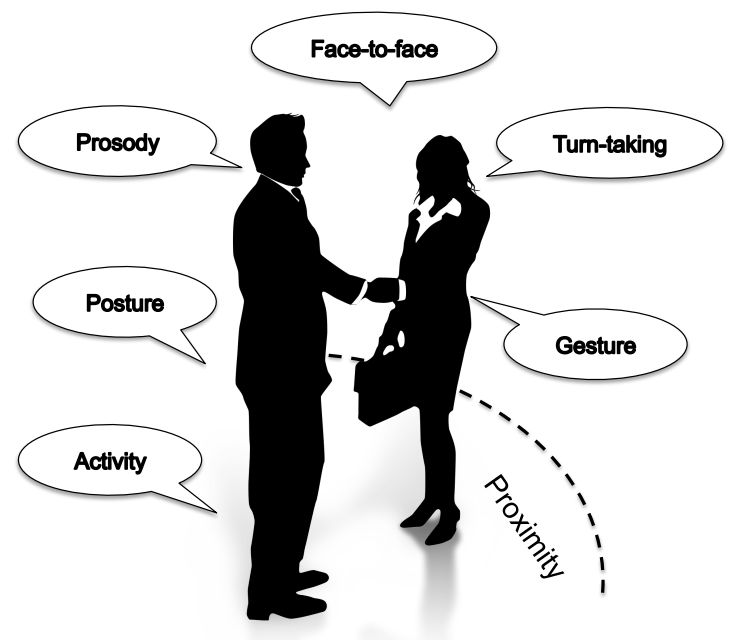

Fig. 4: Behavioural cues emitted during a social interaction.

with Kernel Density Estimator (KDE) model for proximity detection. An external accelerometer was attached to a user's chest to monitor his speech activity by performing spectral analysis of the signal it produced.

Discussion. In order to achieve higher accuracy and robustness in various environments, researchers combined various modalities. Virtual Compass [Banerjee et al. 2010] combined WiFi and Bluetooth to improve distance estimation and provide relative spatial arrangement detection. However, the simultaneous utilisation of these interfaces cannot be utilised for continuous sensing due to high energy consumption and lack of ad-hoc communication in current available WiFi on COTS mobile phones. For further improvement of social interaction detection on smartphones, [Matic et al. 2012] designed a work that provides a relatively accurate approach. As Fig. 3b indicates, this method is able to identify correctly that (A) and (B) are socially interacting while (C) and (D) are not. They tracked user's orientation and logged speech activity through an accelerometer attached to a user's chest. Yet, the smartphone was in a fixed body position and the external accelerometer was intrusive as it was placed on user's chest.

\subsection{Apposition of Social Interaction Detection Approaches}

In this section, detection methods for social interactions among people were surveyed by presenting different approaches that researchers have developed to tackle this problem. It is important to understand the strengths and the limitations of each method. As mentioned, the single modality works provide the benefit of low complexity, unobtrusiveness through limited constraints regarding the wearing position and the lack of external hardware. However, the approach is characterised by a large amount of false positive errors, which depending on the application could be neglected or require tackling. In case this error is not acceptable, a multi-modal approach is more suitable that incorporates the user's facing direction and conversation detection. Although, these multiple modalities provide additional information to tackle the social interaction detection problem, researchers may consider the accumulated error introduced by each modality. Through efficient fusion of several modalities, the error of each individual modality may cancel each other out, driving the system to a less erroneous approach.

Despite the numerous works on attempting to detect social interactions on mobile phones, to our knowledge currently there is no tool that may be utilised in a real-world environment, without any constraints and with minimum intrusiveness. Ideally the framework may not require any firmware modification. Also, it would be able to be deployed on off-the-shelf smartphones providing a realistic and robust accuracy in a real-world environment, depending on the target application given only the integrated sensors of the device. Finally, both approaches are characterised by a trade-off among user friendliness, system complexity and accuracy [Madan and Pentland 2006] that should be considered by researchers depending on the needs of each application.

\section{BEHAVIOURAL CUES EXTRACTION}

Social interaction detection provides personalised information about who is interacting with whom. As Fig. 4 shows, during a social interaction interlocutors emit cues such as spatial arrangement, posture and gestures indicating social signals such as intimacy, interest, mirroring etc. Acquiring this knowledge leads to the next stage of mining human behaviour on mobile devices, which is the extraction of behavioural cues. In this process, data obtained by sensing procedure are pre-processed or classified through a machine learning technique to retrieve some features that will be utilised in the next stage, the inference of social signals. 


\subsection{Auditory}

In SSP, literature has focussed mainly on extracting social signals from audio and visual data. This fact procures a reasonable indication regarding the importance of behavioural cues extracted from audio data. This section aims to provide a brief overview of the techniques applied on acoustic data recovered from ambient sensors and especially from a mobile device's microphone. The techniques are categorised based on the type of features extracted. The same classification was performed in [Vinciarelli et al. 2009]. Thus, following and extending that taxonomy for techniques applied on mobile devices was considered as a reasonable continuity. It is noted that social signals refer to non-verbal communication signals emitted when a person is socially interacting [Pentland 2007]. Hence, natural language processing is excluded from Mobile SSP as it considered a separate field.

5.1.1. Prosody. This behavioural cue provides information about the characteristics of a person's voice when socially interacting i.e. phrasing, stress, and intonation [Selkirk 1995]. To infer about the prosody of a person's voice on mobile devices, literature has focussed on extracting certain features. Razak [Razak and Abidin 2005] extracted prosodic features such as energy, linear predictive coding (LPC) coefficients, duration, pitch and jitter of each recorded frame. VibeFones [Madan and Pentland 2006] focussed on pitch, amplitude, mean energy, frequency of the fundamental format and spectral entropy. AMMON [Chang et al. 2011] calculated zero crossing rate (ZCR), root mean square, frame energy, pitch, harmonics-to-noise ratio and Mel-Frequency Ceptral Co-efficients (MFCC). StressSense [Lu et al. 2012] reported that pitch and its derivatives were the most informative features, followed by jitter, spectral centroid, high frequency ratio, speaking rate and MFCCs. In SoundSense [Lu et al. 2009] authors extracted ZCRs, low energy frame rates and other spectral features which were fed in a J48 decision tree [Quinlan 1993]. The most recent work SocioPhone [Lee et al. 2013b] calculated prosodic features through pitch, energy, loudness, rhythm and spectral features (formants ${ }^{3}$, bandwidths, spectrum intensity).

Discussion. The optimal prosodic feature set varies based on the target application. [Razak and Abidin 2005] evaluated different prosodic feature sets. Those that included LPC coefficients had the best performance while the set with speech energy, duration, pitch and jitter performed worst. Authors did not apply any feature reduction technique on the training data, based on various factors such as information gain to retrieve the most informative feature set [Hall and Holmes 2003]. VibeFones [Madan and Pentland 2006] require a long-term analysis to derive standard deviations of the features and do not describe the process of concluding to the particular feature set. $A M M O N$ [Chang et al. 2011] showed the performance improvements achieved when combining prosodic features with glottal timings ${ }^{4}$. However, the system was evaluated off-line on datasets created in constrained environments while the performance in real-world situations was not provided. StressSense [Lu et al. 2012] selected a particular feature set based on information gain. Authors did not provide any quantitative analysis of energy consumption of the system as they extract cumbersome features including MFCC. It should be noted that although MFCC improved the accuracy of the system, these features allow rebuilding of speech segments and further natural language processing. This fact induces some privacy issues as opposed to features such as pitch, speaking rate, jitter which do not allow derivation of speech segments. SoundSense [Lu et al. 2009] focussed on features that are not affected by the volume; in spectral features they removed DC components. To preserve users' privacy they performed data processing on the device and then discarded any raw audio readings. SocioPhone [Lee et al. 2013b] is able to cope with ambient noise distributed uniformly to nearby devices but does not incorporate any on-body position detection mechanism of the device to discard positions such as bags that degrade the quality of raw sound signal.

In literature, many works inferred that features such as pitch and its statistics were considered as the most informative features. Additionally, features such as speaking rate, MFCCs, energy and spectral characteristics were also included in the process of detecting the vocal prosody of a user. The spectral-based features constitute a common ground in various works and especially formants, bandwidths and intensity. The disadvantage of these features is the requirement of transforming the time-based values into frequency based values before every inference, which induces additional computational burden. Only a specific set of the twenty MFCCs are mainly selected during the inference process depending on the classification target. Before including MFCCs in the feature set, a designer may consider the accumulative burden of these coefficients due to a computational demanding extraction procedure.

5.1.2. Conversation vs Silence. During a social interaction, speech and silence operate as regulators of a conversation emitting social signals such as consensus, rejection and reveal interlocutors' social behaviour including their emotions [Koudenburg et al. 2011]. One of the well-known and widely-used techniques to infer conversation existence was presented by Basu [Basu 2003]. It specified a linked Hidden Markov Model (HMM) with three features: non-initial maximum of the normalized noisy autocorrelation, number of autocorrelation peaks, and normalised spectral entropy. The first layer of the model infers regarding voice existence and the second layer speech occurrence. This technique was adopted by [Choudhury and Basu 2004], Vibefones [Madan and Pentland 2006], StressSense [Lu et al. 2012], MeetingMediator [Kim et al. 2008], [Wyatt et al. 2011]. Another technique widely used

\footnotetext{
${ }^{3}$ Formant in a vocal signal is the accumulation of acoustic energy close to a certain frequency.

${ }^{4}$ Glottal timings refer to the air flow variations produced during speech.
} 
by systems such as SpeakerSense [Lu et al. 2011] and Auditeur [Nirjon et al. 2013] is to calculate the ZCR of an audio frame and then apply a classification method to infer if the segment contains speech [Saunders 1996].

Matic [Matic et al. 2012] inducted a privacy-oriented approach that incorporated an accelerometer on a user's chest. By analysing the sum of the power spectral densities, computing integral and mean of the frames $(80-256 \mathrm{~Hz})$, and feeding it to Naïve Bayes with KDE [John and Langley 1995], they were able to detect when the user was speaking. CoenoFire [Feese et al. 2013], detects speech through Long-Term Signal Variability (LTSV) presented in [Ghosh et al. 2011]. Also, AutoSense [Ertin et al. 2011] utilised a Respiratory Inductive Plethysmograph (RIP) in order to compute lung volume and breathing rate from which they detected conversation existence.

Discussion. For detecting conversation existence or absence through a mobile device, research focussed on audio-based and accelerometer-based data. The conversation detection methods based on microphone data funnel on [Basu 2003] or through some pre-processing steps they train a Gaussian Mixture Model (GMM) that identifies (non)conversation segments. [Basu 2003] is a well-established approach for detecting speech in raw microphone data, which achieves less than $10 \%$ error estimations at $6.4 \mathrm{~m}$ even with increased interpersonal distance. [Nirjon et al. 2013] brought together the most popular pre-processing steps in order to train a model for conversation and silence detection. A post-processing step may be applied to add time dependence through an HMM. Among the preprocessing steps utilised, performing Fast Fourier Transform (FFT) and extracting MFCC features constitute the most energy consuming processes among the state-of-the-art techniques as opposed to ZCR which is a simple and robust feature. [Matic et al. 2012] and AutoSense [Ertin et al. 2011] induced two privacy preserving approaches ${ }^{5}$ for speech recognition through accelerometer (93\% accuracy) and RIP data (over 87\% accuracy), both evaluated in real-world situations. Although [Matic et al. 2012] achieved around 10\% error rate in real-world environments, it is prone to coughing and various mean of transportation that confers vibrations. Additionally, attaching an external sensor on user's chest is considered intrusive. CoenoFire [Feese et al. 2013] focussed on LTSV, which is suitable for noisy environments but is not able to discriminate speech among various users. Finally, classifying conversation existence is a process that can be applied on mobile phones as shown in literature, nevertheless including energy consuming pre-processing steps will increase the computational burden.

5.1.3. Turn-taking and Vocal Outbursts. In linguistics, turn-taking refers to the process of exchanging speech turns during a conversation, including speech overlap, showing the willingness of a person to continue a conversation [Schegloff 2000]. The occurrence of non-linguistic vocalisations i.e. vocal outbursts, provide additional information about interest, boredom, willingness to continue a conversation etc. among the interlocutors [Schrder 2003]. Given a set of features, turn-taking detection is identified mainly by training a GMM for each speaker through an Expectation Maximisation (EM) algorithm, to allow classification of the most probable speaker for a certain speech frame. This process is called speaker recognition and is harnessed to understand between which people turn-taking is occurring. [Miluzzo et al. 2010] developed an on-line speaker diarization system that in a distributed manner infers interlocutors turn-taking through the above described typical speaker recognition pipeline. In [Aran and Gatica-Perez 2011], authors perform speaker diarization ${ }^{6}$ and then they calculate three types of turntaking features: a) independent (speaking length, number of speaking turns, turn duration statistics), b) relational (interruptions, order, centrality), c) meeting (number of silent moments, overlapped speech). A similar approach was followed by SocioPhone [Lee et al. 2013b]; instead of meeting features they incorporated interaction features that included the duration of speaking and non-speaking turn. For detecting vocal outbursts VibePhones [Madan and Pentland 2006] considered the distribution of utterance length i.e z-score.

Discussion. Understanding turn-taking in an audio data sequence, requires the execution of speaker recognition and then identification of the segments with speaker change or overlapping. The features selected are the same utilised in conversation detection. A great deal of attention should be paid in selecting the optimal dataset with respect to the application context in order to achieve high accuracy and robustness in the inference process. A certain model is trained for each speaker, inducing the requirement of a speaker models library if the system extracts information from all the interlocutors. In the case of adding a new user to the system, a model has to be trained especially for this user and incorporated to the library. Then a Maximum Likelihood algorithm is applied to identify the most likely model. The speaker diarization, in most cases, is executed off-line where all audio segments have been logged and categorised to each speaker such as [Aran and Gatica-Perez 2011]. For on-line execution, the process requires a connection with the centralised library in order to identify the speakers and then log information about turn-taking. SocioPhone [Lee et al. 2013b] performs turn-taking detection on-line, it achieves its highest accuracy when the number of devices is equal to the interlocutors and the devices are placed on a meeting table. When the number of mobile devices is reduced, or the device is placed in a bag or in trousers pocket, the accuracy is degrading. In contrast, [Miluzzo et al. 2010] was able to maintain similar accuracy even when the number of smartphones was reduced but the recognition accuracy was degraded in short-term turn-takings. Loading all speaker models on the device will increase the computational burden, energy consumption and will degrade user experience. In contrary, off-loading this computation burden to the cloud will induce a certain communication cost

\footnotetext{
${ }^{5}$ [Matic et al. 2012] utilised accelerometer data and AutoSense [Ertin et al. 2011] sensed lung volume and breathing rate, thus they are considered privacy preserving approaches as they do not focus on audio data that allow natural language processing.

${ }^{6}$ Speaker diarization refers to the process of speaker recognition followed by clustering with respect to each speaker.
} 
in terms of energy consumption and cost increase in users' data plan. Having performed speaker diarization, statistical analysis such as speaking time, number of turns etc. can provide valuable information about the sociability and the overall social behaviour of the user. Regarding vocal outbursts VibePhones [Madan and Pentland 2006] utilised the z-score of utterance length which is an easy to extract feature but requires the knowledge of mean and deviation of the population to compute the particular feature.

5.1.4. Speech Activity. Another auditory cue is speech activity which includes various derivatives of the time a person is talking in a conversation i.e. accumulated speech time, speaking frequency etc., indicating social behaviours such as sociability and dominance. Vibefones [Madan and Pentland 2006] detects user's activity in voice, by performing initially speech recognition and then calculates the z-score of the time the user was speaking. In CoenoFire [Feese et al. 2013], having detected speech in audio data, they compute the speaking time for each user which indicates the speech activity of each participant.

Discussion. Speech activity is a feature that can be derived from previous auditory cues. Literature has extracted this cue by initially detecting voice and speech through the aforementioned techniques. Further, by inferring the speech segments that belong to the user, through a personalised speech detection model, an accumulation of the speech windows is performed through statistical analysis. This accumulation refers to the computation of speech frequency, overall speech time etc. Vibefones [Madan and Pentland 2006] considered z-score as measurement of speech activity. It provides the probability of a person speaking with respect to the rest of the speakers but requires all users' speech segments to perform the computation. CoenoFire [Feese et al. 2013] performed a more light-weight speech detection method but considered only the overall speaking time. The initial process of personalised speech detection induces the computational burden, in contrast to speech activity inference which is mapped to statistical analysis on the speech segments of a specific user.

5.1.5. Auditory in essence. When Pentland [Pentland 2007] introduced social signals, he proved the applicability of the domain based on behavioural cues that were extracted from audio data. This induction may undermine the importance of these types of behavioural cues but also may indicate how informative they are. [Kendon 1990] refers to conversation as an occasion of social interaction which highlights the correlation of the terms in context of human behaviour. For that reason a great deal of non-verbal communication may accompany the spoken linguistics. As shown, features related to the interlocutors' voice can provide significant and informative features. However, some feature extraction processes introduce a considerable amount of computational burden and energy consumption but there are alternatives that can be utilised. A considerable amount of research has been conducted based on audio data. Thus, applying these techniques on mobile devices and in real-world environments will constitute a stable and robust solution for social behaviour inference, with a proportional cost in computation and energy.

\subsection{Physical Activity}

After the incorporation of accelerometer sensor on mobile phones, initially by Nokia and later on by Apple which led to the evolution of smartphones, physical activity became a popular behavioural cue [Choudhury et al. 2008]. This provides additional contextual information allowing the reduction of false positives in situations such as stress detection. Furthermore, it may be utilised as an optimisation process e.g. to discard data under the assumption that when the user is running, he is not socially interacting. Most approaches focussed on computing statistical features showing how active the user is but also what kind of activity the user was performing, such as standing, walking or running. In order to classify the activity the user is performing, researchers mainly train an activity detection model for each category that requires identification. In the next subsections state-of-the-art techniques are presented, that have been developed for measuring how active users are and in which activity state they are in.

5.2.1. Movement Activity. One of the cues a person is conveying when socially interacting, is movement activity e.g. small but noticeable activity when being in standing position, which may reveal social behaviours such as stress. A low complexity and robust method was presented in Social fMRI [Aharony et al. 2011]. The authors retrieved $3 \mathrm{D}$ accelerometer data from mobile phones, for 15 seconds every 2 minutes and then they computed a vector with the frame's magnitudes. They then calculated the variance for each second of the frame which was utilised as a ranking mechanism to classify the user's activity as still, moderate or high based on a threshold.

In Jigsaw [Feese et al. 2013] authors extract movement activity by retrieving information from the accelerometer of the mobile phones. First, by calculating standard deviation of a moving window and then based on a threshold they classify the segment as active or non-active. In addition, they compute the movement intensity through the median magnitude of the linear absolute acceleration. It should be mentioned that these features are focussed on fire-fighters, who work in an intense environment. Berke [Berke et al. 2011] utilised the accelerometer and barometer sensors to detect users' activity and the time spent performing the specific activity given a certain weight. Based on the importance of the activity they provided an analogous weight to the time a user spent in performing a certain activity. Muaremi [Muaremi et al. 2013] computed activity movement of a user based on accelerometer and GPS data of the mobile device. From 3D accelerometer data they calculated the magnitude and 
further mean and variance values. From GPS data the amount of locations a user visited and the travelled distance, were computed.

Discussion. Movement activity detection has been tackled with several methods, mainly by computing statistics such as variance of a window over raw 3D accelerometer data and then applying an empirical threshold. Social fMRI [Aharony et al. 2011] is one of these methods, which can be utilised easily through the Funf [Funf 2011] open-source sensing framework that performs the procedure as a pre-processing step. A similar technique was implemented in Coefire [Feese et al. 2013], in [Berke et al. 2011] where authors included a time-dependent factor and [Muaremi et al. 2013] incorporated a location-change magnitude feature. Given the energy consumption of the aforementioned sensors, the most suitable approach for continuous sensing applications is the extraction of accelerometer statistics which is a lightweight and reliable procedure [Hoseinitabatabaei et al. 2013]. Although, the location incorporation provides an additional measurement and parameter, it is based on GPS; a high energy consuming sensor which cannot be used for long-term sensing applications or should perform sensing in an efficient manner. However, a less accurate approach may be utilised such as GSM localisation techniques to provide coarse grain location estimation. In essence, detection of activity movement for continuous sensing applications, may focus on less power "hungry" sensors such as the accelerometer, and extract information through low complexity statistics suitable for the majority of social behaviour applications.

5.2.2. User's Activity State. Activity recognition provides important information for understanding the context in which a social interaction is taking place. Also, it allows researchers to create more accurate and reliable techniques for social behaviour understanding based on a specific activity state. There has been a considerable amount of research in order to detect the activity that a user is performing based on sensor data retrieved from mobile phones. In this part, we mention only the most important works analytically, to provide an overall understanding to the reader regarding the process of activity recognition. An extensive survey on activity recognition through body worn sensor is presented in [Bulling et al. 2014].

Yang [Yang 2009] presented an activity recognition approach that utilises orientation independent features for vertical and horizontal components of accelerometer data. He computed the mean, standard deviation, ZCR, interquartile range, $75 \%$ percentiles, spectral entropy and entropy of both components and their cross correlation. The features were forwarded to a decision tree [Quinlan 1993] leading to $90 \%$ accuracy for sitting, standing, walking, running, driving and bicycling. Also, he proposes an approach to reduce data over-fitting, that combines KMeans clustering (a cluster for each activity) followed by an HMM-based Viterbi algorithm to leverage historical data.

A lightweight approach for detecting users' state is presented in [Wang et al. 2009], that is based on the standard deviation of the magnitude of accelerometer data. It does not rely on the device orientation and achieves above $70 \%$ accuracy. Initially authors retrieve $\mathrm{N}$ (windows size) number of measures from the accelerometer and convert them to magnitude time series. Then from the produced signal, they extract entropy, power, value and amplitude of the highest magnitude frequency, weighted mean/variance of the top-t highest magnitude frequencies (weighted by amplitude). For classification they perform off-line supervised training for each user a C4.5 decision tree model (70\% accuracy) by utilising accelerometer data which are labelled with respect to the activity that is performed. Feature extraction and classification is executed in real-time [Srinivasan and Phan 2012].

Jigsaw [Lu et al. 2010] performed activity recognition and divided the process in four stages: a) calibration, b) pre-processing, c) feature extraction, d) activity classification. In the first stage, authors calculate the offset accelerometer parameters for the specific device through a linear least square estimator. The pre-processing stage includes outlier removal and projection of the accelerometer data to earth's coordinates. Then, time and frequency domain features are extracted based on mean, variance, mean-crossing rate and spectral analysis. Finally, a decision tree is created followed by a sliding window smoothing method achieving $91.64 \%$ accuracy for cycling, vehicle, running, stationary and walking. Based on this method, they provided an extension which through a crowd-sensing technique creates a personalised model for detecting activity in a population of users [Lane et al. 2011].

Seiter [Seiter et al. 2013] utilised mobile phones that incorporate accelerometer, barometer and GPS to understand the level of pain in a patient based on his activity. Authors concluded that based on $40 \%$ of pain relief, $10 \%$ degradation was detected. It should be mentioned that the study was conducted on only one person. Also, [Yan et al. 2012] presents an adaptive activity recognition method that leverages mean, variance, entropy and energy (FFT) of a frame, in order to compute the confidence of an activity and then apply the appropriate pair of sampling frequency and feature set. Furthermore, $P B N$ [Keally et al. 2011] describes an approach with multiple sensors deployed on the user. For each sensor a unique classifier is trained and an overall classifier based on Adaboost [Freund and Schapire 1997] computes user's state on the mobile phone.

Discussion. For estimating the user's activity state, research focussed mostly on extracting statistical and spectral features from a window of accelerometer samples and based on these features train a C4.5 decision tree. This constitutes a simple and straight forward approach, with lightweight feature extraction and classification model, ideal for mobile devices. Regarding the features, some exceptions are the processes of computing FFT and spectral entropy which add a considerable burden to the device. Jigsaw [Lu et al. 2010] sensing framework managed a comparable accuracy $91.64 \%$ on mobile phones. Both approaches could provide a realistic solution in order to 
tackle the certain problem. Achieving similar accuracy without the need of extracting the burdensome features, could constitute a significant challenge. In $P B N$ [Keally et al. 2011] training a specific classifier for each on-body position and combining those in a hierarchical model, provide an accurate outcome but limits the pervasiveness of the system in daily lives. In conclusion, incorporating users' activity state recognition allows the creation of specific social behaviour inference models for different activities. These activity-dependent models led in reducing the error in social behaviour inference.

5.2.3. An outline of physical activity. Detection of users' physical activity on mobile devices is an area that has triggered the interest of scientists mainly from the point of accelerometer integration in COTS smartphones. The works related to physical activity are highly correlated to the detection characteristics of user's movement. This includes qualifying that a user is more active than another and classifying users' current activity state such as standing, walking, running. Both fields provide important information about the user and his behaviour but also about the context in which he is. To summarise, movement activity detection is a relatively simple and lightweight process that is supplied by several sensing frameworks as a pre-processing step. In contrast, users' activity state has a great spectrum of inference techniques that adds a notable amount of computational burden and demands model training for pre-known states. However, user's activity state redounds significantly in detecting the context in which the social interaction is taking place and should be included in the inference process.

\subsection{Gesture and Posture}

Gesture and posture are two means through which people emit signals during social interactions. A noticeable difference exists among several cultures but both cues convey important information about the social situation, attitude, relationship of the participants etc. In SSP, gesture and posture inference is performed through video recordings, in which researchers detect certain body parts of the participants. Then by tracking these points, they train classifiers that infer about various gestures and postures [Vinciarelli et al. 2009]. In this section we present techniques developed to detect various gestures and user's posture through mobile phones while in some cases with the incorporation of external sensors.

5.3.1. Gesture. There is lengthy research regarding gesture recognition through several means such as video and body-worn sensors. Literature focussed on utilising smartphones' integrated sensors but also incorporating external hardware. Regarding the inference, a popular approach is to train a Markov Model for each of the targeting gestures and based on the confidence that each model produces, the highest is selected.

PEYE [Hua et al. 2007] focussed on detecting simple motion gestures on mobile phones by utilising the camera and recording small videos. These are split into sequential images from which they extract small rectangles that are tracked through an adaptive block matching approach in order to understand the device's movement and further user's hand gesture, with $12.86 \%$ minimum matching error. e-Gesture [Park et al. 2011], proposed to train a generic HMM classifier for each gesture, which retrieved data from a hand-worn sensor and forwarded them to a smartphone. The accelerometer and gyroscope data are segmented in an adaptive manner based on gesture change through their magnitude. Then, they are forwarded to an adaptive (Maximum Likelihood Linear Regression for model update) or multi-situation HMM (one HMM for each situation: ride, stand, walk, run) for gesture recognition in four different situations, achieving $84.6 \%$ and $94.6 \%$ accuracy respectively.

Authors in [Junker et al. 2008] extracted pitch and roll of a user's hand from body-worn sensors. By applying pre-processing mechanisms and an HMM they were able to identify user's gestures with $97.7 \%$ accuracy. The pre-processing step was based on SWAB [Keogh et al. 2001] that performs segmentation and approximation on time correlated data. These segments were grouped based on resemblance and the ones with the lowest similarity were selected. $u$ Wave [Liu et al. 2009] introduced Dynamic Time Warping (DTW) that performs adaptive gesture classification through sensed accelerometer data based on only one training sample and achieved $98.6 \%$ accuracy on 8 gestures. A pre-processing step is preceded, that performs quantization on raw accelerometer data to remove noise and reduce the size of the data.

As an improvement of DTW, [Wu et al. 2009] presented a frame-based descriptor and multi-class Support Vector Machine (SVM) that was able to detect 12 distinct gestures with 95.21\% accuracy. Myo [Myo 2013] is a newly developed wearable armband that is able to perform gesture and motion control. It detects the muscle movement of the user's arm (Electromyography) and transmits that information through Bluetooth to another device such as a mobile phone. It should be noted that $M y o$ is a commercial product and its accuracy is not provided. For further information about the analytical works that have been done and proposed techniques in the area of gesture recognition we refer to an extensive survey [Mitra and Acharya 2007].

Discussion. In essence, PEYE [Hua et al. 2007] performed mainly device movement recognition through video recording. This may be replaced by orientation sensor readings due to lower power consumption of sensor and process. e-Gesture [Park et al. 2011] proposed an adaptive method that continuously learns based on user-labelling but has increased computation due to learning and may not perform well due to data diversity. An adaptive model for each situation (e.g. standing, walking etc.) may achieve higher accuracy as it will create an activity-dependent classification. The multi-situation approach had the highest accuracy, but requires training of known situations. [Junker et al. 2008] focussed mainly on detecting the type of activity the user was performing while gesture recog- 
nition was deficient due to lack of a garbage model; a model that infers if none of the target classes are detected. $u$ Wave [Liu et al. 2009] claimed high accuracy (98.6\% for 8 gestures) through their adaptive approach. However, it should be noted that it is a user-dependent method which must be personalised to each user and there is a requirement for tracking the device orientation in case it is tilted. Myo [Myo 2013] provides a pre-defined detectable gesture collection but a developer is able to add his own. In overall, both HMM and DTW methods achieved high accuracies. Because every person is different, a personalised approach will achieve highest accuracies. However, this constitutes a trade-off due to the requirement of additional training and user dependence. Also, in $e$-Gesture [Park et al. 2011] the utilisation of a limited amount of training data, leads to the computation of non-optimised thresholds as a result the adaptive methods may achieve lower accuracy. Overall, approaches that utilise inertial data are more suitable than the video-based approach due to mobility and energy restrictions, however adaptive sensing and inference may be required.

5.3.2. Posture. A person's posture is divided into head and body posture. Both produce non-verbal communication during a social interaction through the tilt of the certain body part. This is a brief description of existing literature for both classes in the following subsections.

5.3.2.1. Head Posture. Being able to detect head posture through a mobile device can provide valuable information about a social interaction such as where the user is facing and if the head is tilted. For example, during a social interaction when people have a common interest or agree on a certain topic they tend to tilt their head to the same direction, i.e. mirroring [Chartrand and Bargh 1999]. Thus, head posture detection is another significant behavioural cue that could be utilised for social behaviour inference.

In SEPTIMU [Hong et al. 2012] an accelerometer and a gyroscope are integrated inside an earphone and utilised the microphone transmission of the headset, to communicate with a mobile phone which was used to infer about the head posture of the user. Smart Pose [Lee et al. 2013a] and [Lee et al. 2012] employed the orientation sensor and the front-camera of the mobile phone to calculate the user's neck angle with respect to the earth's coordinates. Initially, the system performs face detection through Android API built-in functionality. It identifies if the user is holding the device in the hand by shake detection (threshold-based) on accelerometer data. Finally, based on the orientation sensor and user's viewing angle with respect to the device, it computes the average neck tilt angle. Another technology that has been developed is Google Glass [Google Glass 2013]; wearable glasses incorporating multiple sensors such as gyroscope, accelerometer and magnetometer allowing to be utilised as non intrusive technology.

Discussion. Research focussed mainly on obtrusive approaches for head posture detection. SEPTIMU [Hong et al. 2012] claim of head tracking provides a simple but obtrusive solution because it requires the user to continuously wear an earphone. Smart Pose [Lee et al. 2013a] constitutes a low complexity and multi-modal approach for head posture detection without any external hardware, it relies on off-the-shelf smartphone integrated sensors but requires the user to hold the device in the hand and also interact with it. Lately, wearable devices constitute a viable solution for accurate and reliable head posture inference.

5.3.2.2. Body Posture. An equally important class is body posture detection that convey social signals such as mirroring and intimacy during a social interaction. While people are interacting they tend to bend towards a person showing a certain level of intimacy while a body slope opposite to the interlocutor may indicate inconvenience. Thus, detecting this type of signals can provide underlying information about a social interaction.

In imWell [Jovanov et al. 2013], a sensor incorporating an accelerometer is placed under the left arm of the user and transmits logged data via Bluetooth to a mobile phone. To detect a different posture, they target identifying transition points. A pre-processing step is applied that computes the standard deviation of 1 second window of accelerometer samples, to remove minor movements. Then, the angle change with respect to vertical position is computed, which determines the upper body posture. Having the standing position of the user as a reference, they categorise the user's body posture based on certain thresholds. CONSORTS-S [Sashima et al. 2008] utilises the average of the accelerometer window of samples from a wireless on-body sensor and based on the device inclination it classifies through decision rules, about the posture of torsos (standing, facing up or down). [Liu et al. 2012] perform body posture recognition by retrieving measurements from mobile device's orientation sensor and especially pitch, to classify if the user is sitting or standing, while the smartphone is in the user's trousers pocket. They allow a margin of error of $20^{\circ}$ around $180^{\circ}$ or $0^{\circ}$ of pitch to infer that the user is standing. With the same error margin around $90^{\circ}$ or $-90^{\circ}$ they estimate if the user is sitting. Another approach that provides information about user's torsos facing direction with respect to earth's coordinates is $u$ Direct [Hoseinitabatabaei et al. 2013] that utilises inertial sensors of off-the-shelf mobile phones.

Discussion. Literature has targeted mainly inertial sensors to estimate body posture. imWell [Jovanov et al. 2013] utilises a very simple technique to identify different body postures. However, it is considered as an intrusive methodology because it utilises an external sensor that is tied around the user's torsos. CONSORTS-S [Sashima et al. 2008] performs rule-based decision on average of accelerometer sample-window. It constitutes a lightweight process regarding the feature extraction and the inference but is susceptible to on-body position changes of the device. So, in order to improve the accuracy of the approach it requires the creation of different rules for each on- 
body position. [Liu et al. 2012] described a threshold based approach on the orientation sensor's pitch. It is easily implementable but is applicable only for the trousers pocket and requires the device to be in a vertical position, thus it does not provide a generic solution. Regarding $u$ Direct [Hoseinitabatabaei et al. 2013], it assumes that the relative orientation between the device and user's body is static. In unconstrained environments the devices are not fully attached to a user's body and are able to move in a certain range. Also, a pre-processing step is required to identify the on-body position of the device. Overall, the extraction of body posture is mainly based on accelerometer data, a relatively low energy consumption sensor, but still requires an adaptive sensing mechanism. Also, the onbody position of the device may be considered as contextual information to target the inference on specific body parts ${ }^{7}$ and discard unrelated positions.

5.3.3. Revealing the methodology. Overall, gesture detection on mobile devices in current literature requires either the user to hold the device in his hand or the incorporation of an external sensor. The integrated accelerometer could be considered as the main source of data, through which gesture specific models can be trained. In posture detection, head tracking solutions are mostly intrusive (video or external sensor) however body posture detection could be implemented with COTS mobile phones. The increased popularity and close body attachment of wearable devices, which connect with smartphones, shows good potential in real-world situations for both gesture and posture detection. A gap identified in literature, is the lack of on-body position detection of the device before performing body posture inference. The on-body position of the device [Hoseinitabatabaei et al. 2014] [Shi et al. 2011] constitutes a necessity in order to accurately compute the posture in real-world applications.

\subsection{Facial Cues}

One of the most expressive parts of the human body that people used to externalise their interest, agreement, disagreement, surprise etc. is the face. This emission of social signals is mainly achieved through facial expressions and eye movement. Thus, providing a detection and quantification mechanism of behavioural cues vented from a person's face is not negligible, while relying on mobile devices.

5.4.1. Facial Expressions. People communicate verbally during a social interaction and in parallel emit social signals also through their faces. Several works in psychology showcase the importance of facial expression in recognising interlocutors' emotions such as valence, arousal, disgust, embarrassment and amusement [Ekman 1993]. In addition, due to this high correlation they claim a high accuracy in detecting these emotions through facial expressions. For a comprehensive literature review of face recognition, the reader is referred to [Hjelmas and Low 2001] [Zhao et al. 2003]. State-of-the-art techniques for detecting facial expressions through mobile phones are presented in the following paragraphs.

Detection of facial expression of a user through a mobile phone is presented in Visage [Yang et al. 2013]. The approach is based on information retrieved from the camera and motion sensors of the device, for face and head pose recognition respectively. Through the data, authors perform face detection [Viola and Jones 2004] (Adaboost-based object detection) inferring rectangle features allowing face tracking. The knowledge of head and face reference points combined with Active Appearance Models [Matthews and Baker 2004] merged texture and shape of the face and allowed them to detect different facial expressions. Performing real-time training and recognition of facial features is presented in [Choi et al. 2011] based on novel non-orthogonal local random basis. According to the authors, this method provides a robust but energy efficient solution for extracting facial characteristics. The features are forwarded to a neural network which performs classification and updates the decision thresholds. They evaluated against six well-known face databases and benchmarked against Principal Component Analysis (PCA) approach [Turk and Pentland 1991].

Another proposed method in order to detect facial expressions on mobile phones is [Cho et al. 2009]. Initially, the authors propose the utilisation of two SVMs, a micro and a macro component. The first layer computes the score of the input image with respect to pre-trained (non-)facial classifier and then a second layer SVM calculates the fiducial points: eye, nose, mouth. The acquisition of fiducial points leads to the extraction of Local Gabor wavelet features through Gini selection method. In [Doukas and Maglogiannis 2010] authors performed face recognition on mobile phones based on eigenfaces [Turk and Pentland 1991], a well-known and established approach. By retrieving an image from the smartphone's integrated camera, they detected the user's eyes. Utilising the eyes' position they were able to mine several facial feature points. By tracking these facial features, a classifier can be leveraged that enables the identification of various facial expressions.

Discussion. Most of the works in facial expression detection were designed, implemented and evaluated in constrained environments, mainly to showcase the applicability of such cumbersome techniques. One of these works was Visage [Yang et al. 2013] that was able to detect facial expressions on mobile phones through a well-established face recognition method [Viola and Jones 2004]. However, the system realisation in real-world environments including the energy consumption was not evaluated, implying its limited applicability in realistic situations. Applying NN approach for classification on mobile phones in [Choi et al. 2011], was proven insufficient. It is worth noting that although PCA with SVM outperforms conventional random basis, it requires training. [Cho et al. 2009]

\footnotetext{
${ }^{7}$ Most common wearing positions include trousers pockets, belt, hand, chest pocket, handbag, backpack [Ichikawa et al. 2005]
} 
tracked users eyes, mouth and nose, which provided some concrete identification points for facial expression detection. During real-time classification they claim an inference cycle of five seconds by the Boosting Naïve Bayesian (BNB) algorithm, with an overall accuracy 75\% for four expressions. [Doukas and Maglogiannis 2010] applied eigenfaces [Turk and Pentland 1991], distance projection and computation, classification on mobile phones. The inference process duration was about 1.2 seconds, which constitutes a reasonable delay for face recognition. However, they have not included any analysis regarding the computational burden and the energy consumption of the approach, which will limit the applicability of the method in real-world situations. Even though facial expression detection constitutes a cumbersome process, the value of this behavioural cue is important.

5.4.2. Eye Tracking. During a social interaction a person conveys non-verbal communication signals also from the eyes. Social signals indicating intimacy, interest, personal relation but also conversation regulation are some of the information emitted through eye contact [Kleinke 1986]. Furthermore, [Vertegaal et al. 2001] induces the ability of predicting interlocutors' attention during a group conversation based on the eye movement. Thus, as eye movement is considered an informative behavioural cue in social behaviour inference, state-of-the-art techniques for eye tracking are reviewed in the following paragraphs.

One of the first works for detecting eye movement was eyeLook [Dickie et al. 2005], where authors attached an Eye Contact Sensor (ECS) [Vertegaal et al. 2002] surrounded by LEDs, on a mobile phone. Through the flashing of LEDs, their reflection was displayed on the user's pupils, logged by the attached camera and then transmitted for off-line analysis. LEDs' reflection was displayed near user's pupils which allowed detection and tracking of his eye. By detecting the movement of the participant's eye, they were able to identify turn-taking among users.

For detecting facial movement and especially eye motion [Bulling et al. 2011] developed custom wearable goggles. These were constituted by dry electrodes, light sensor, accelerometer and were connected through a wire to a digital signal processor (DSP) and a data storage. Based on the application they proposed alternative methods, such as electrooculography (EOG) or camera recordings, to detect eye movement. EOG is performed through dry electrodes of the goggles that are attached to participant's face. Authors depict six main feature categories from which they compute various statistics and signal characteristics; a) saccades, b) fixations, c) blinks, d) microsaccades, e) vestibulocular reflex, f) smooth persuit movements. It should be noted that the authors utilised only saccade, sequence, blink and fixation features. By triggering the interest of a participant wearing the goggles, these features are extracted and a model is trained which is applied later on to identify certain eye movements.

In order to create an eye-controlled mobile phone, [Pino and Kavasidis 2012] utilised the front camera of a smartphone for achieving eye tracking. The system takes sequential pictures from the user. Then, it performs a Haar classification that identifies features in a rectangular space through summing the intensity of the pixels. The method detects two similar spaces of the picture and classifies it as the eyes. Having detected the user's eyes, they utilise the CAMSHIFT [Bradski 1998] algorithm to keep track of their position in upcoming images. To transform the detected eye position to the device's display coordinates, they compute the centroids of the rectangles and then they apply the Starburst algorithm [Li et al. 2005] for tracking.

Discussion. A common practice for extracting facial cues is defined by detecting initially fiducial points [Turk and Pentland 1991] [Belhumeur et al. 1997]. Eye positions are some points which may be leveraged for tracking a person's eye movement. Regarding the aforementioned works, eyeLook [Dickie et al. 2005] is based on an obtrusive mechanism that requires detecting the flash reflection near the eye pupil which is additionally prone to daylight reflections. [Bulling et al. 2011] tried to deviate from the main visual-based approaches by utilising a EOG attached to muscles surrounding the eye. This is a less computational consuming approach that provides a raw signal indicating the muscle movement, allowing detection of eye activity. Although, the method requires specific glasses with integrated dry electrodes, the reduction of the complexity is noticeable regarding the video based approach. Furthermore, [Pino and Kavasidis 2012] also focussed on a video-based eye tracking method. By applying a modified Haar feature extraction and classification they were able to achieve a speedy inference of eye points in an efficient manner. The classification process was only initiated when a certain movement threshold was overcome. Finally, performing eye tracking requires tackling some key challenges including a) the high computational power required for retrieving information from visual data and b) the difficulty in detecting the eye pupil in a sunny outdoor environment that is characterised by brightness fluctuations and saturation [Drewes et al. 2007] c) the utilisation of ubiquitous and non-intrusive sensors for retrieving data that will allow the inference.

5.4.3. Facial Cues at a glance. Face is a very descriptive part of the human body during social interaction in terms of social signal conveyance. However, the capability of detecting these behavioural cues on mobile devices constitutes a great challenge. Along with the computationally complex process of detecting facial cues other provocations arise including the intrusiveness of the system, the applied training data but also the real-time on-device classification. Detecting facial cues is stemmed by identifying several fiducial points of a person's face, including the mouth, nose and eye. In many cases the methods include a pre-processing step of detecting these parts of the face and then utilise these points for classifying facial expressions and monitoring eye movement.

Overall, identifying facial cues i.e. facial expressions and eye tracking is a burdensome process, especially for constrained devices such as smartphones. As shown, there are works that have managed to execute these computationally demanding processes on mobile phones. However, they do not provide a concise energy consumption 
and computation burden analysis which will indicate the applicability in continuous sensing and inference applications. SociableSense [Rachuri et al. 2011] is a system that decides based on computational requirement to perform the inference on-device or at a cloud infrastructure. Applying a distributed inference adaptation model such as SociableSense, combined with an adaptive sensing technique based on the context is a viable solution for performing such burdensome processes on smartphones. Finally, wearable technologies such as Google Glass [Google Glass 2013] constitute a promising approach for real-world applications. However, energy consumption is still a great challenge in continuous sensing systems.

\subsection{Environment and Space}

Equally important behavioural cues are space and the environment in which a social interaction is taking place. According to psychologists, the interpersonal distance and the spatial arrangement of the interlocutors provide a large amount of information about their social relation, their intimacy but also the probability of people are interacting in multi-personal interactions [Goffman 1963] [Hall 1966].

5.5.1. Interpersonal Distance. In psychology, proxemics is an area that has been exploited for many years, starting from the work of Edward T. Hall [Hall 1966]. In this work, Hall following the social behaviour among animals, defined some imaginary concentric circles around each person during a social interaction, which indicate the type of relationship among the people. Interpersonal distance is a significant element of social interactions, not only to detect if people are interacting but also to estimate their relationship.

5.5.1.1. Sound. The most promising approach for distance estimation through sound is BeepBeep [Peng et al. 2007]. It is based on ToA without the requirement of time clock synchronisation among the devices. Each of the devices sent out an audible Beep sound and logs its own sample and the remote sound. The device continues to record until it receives the remote Beep. Then, they exchange the standby time and compute the interpersonal distance from the number of samples recorded and the time required to receive them. An extension of BeepBeep is [Liu et al. 2013b] in which the authors develop a transmission scheme and apply an adaptive ToA mechanism to improve the accuracy of the system. Also, Whistle [Xu et al. 2011] is an approach akin to BeepBeep but relies on TDoA by recording the sound from multiple devices and performs the computation at a centralised point. A recent work called RF-Beef [Uddin and Nadeem 2013] combined the methodology of BeepBeep with RF interface to apply TDoA by sending initially a Beep sound followed by an RF beacon. A ToA-based scheme is introduced in [Liu et al. 2013c] that uses a speaker and a mobile phone's microphone to perform distance estimation. [Filonenko et al. 2013] exploited and developed a mechanism for estimating the distance based on TDoA among devices, by producing ultrasound through COTS mobile phones.

Discussion. A different modality for distance estimation, the sound, was considered in some approaches in order to be able to apply techniques that are difficult to deploy on mobile phones such as ToA, TDoA and AoA. BeepBeep was the first work that was able to leverage these types of techniques (ToA) on mobile phones based on sound. By exchanging the time duration, there was no need to perform clock synchronisation among the devices. The technique was applied between two devices only. Following this approach, different works used other techniques such as TDoA or combined them with RF signals. As claimed, they are able to achieve a fine-grained distance estimation among the devices. However, the sound based methods are prone to relative orientation of device and user with respect to the interlocutor. The majority of these approaches utilise audible beacons that are not suitable for ubiquitous usage. [Filonenko et al. 2013] claimed to have achieved the transmission of ultra-sound through COTS mobile phones. For the appliance of TDoA, there is a requirement for speaker array deployment at the environment in order to calculate the time difference between arrival of the beacon at the two speakers. This increases the intrusiveness of the system.

5.5.1.2. RF Interfaces. Interpersonal distance estimation through RF-based technologies (e.g. RF, Bluetooth, $\mathrm{WiFi}$ ) constitutes a common approach due to its easiness in development and implementation. Researchers have developed various techniques to estimate interpersonal distance among users in a coarse-grained and fine-grained manner.

Coarse-grain. A commonly used method to detect if people are in vicinity is to utilise the Bluetooth interface. This is available in the majority of todays mobile devices. By performing an enquiry scan process, a smartphone retrieves discoverable nearby devices. This approach takes into consideration every device that is in the range of Bluetooth radius $(\sim 10 \mathrm{~m})$. It is not affiliated with any intelligence to mine more specific information about interpersonal distance; only details such as the identifier and timestamp are logged. One of these works was Serendipity [Eagle and Pentland 2005] in which the author developed BlueAware framework for mobile phones to log the Bluetooth identifier and current timestamp. [Antoniou et al. 2011] deployed Bluetooth dongles inside a building and through Bluetooth discoverable mobile phones they were able to detect if users were in vicinity. CenceMe [Miluzzo et al. 2008], Friends and Family [Aharony et al. 2011] [Staiano et al. 2012] [Singh et al. 2013a] [Bauer and Lukowicz 2012] [Efstratiou et al. 2012] are other examples of works where they utilised simple Bluetooth discovery to infer if users were in vicinity. Unlike previous approaches, PeopleTones [Li et al. 2008] leveraged cell tower readings to estimate if the users are nearby in a larger scale, claiming an error around $322 \mathrm{~m}$. 
Discussion. As noted before, the most common approach for distance estimation on mobile device is through RF-based technologies. In coarse-grain distance estimation based on the targeting device, researchers focussed on Bluetooth discovery or GSM localisation. On one hand, due to the popularity and implementation simplicity of Bluetooth discovery, it constitutes a widely used method when conducting research into social behaviour. It comprises only of the discovery of nearby devices and logging their BTIDs including the timestamps. There is no processing or inference required, thus if the induced error in distance estimation is acceptable for a certain type of application, this method may be preferred. On the other hand, a large amount of people set their devices on non-discoverable mode or disable the Bluetooth interface of their smartphones, making the coarse-grain distance estimation non-applicable. Nevertheless, the range of Bluetooth introduces a large amount of error, e.g. two people may be in different rooms, but in through this method they are considered close enough to interact. These interpersonal distance estimation techniques rely on the assumption that when devices are in vicinity then their users are as well. However, in real-world situations this assumption is not always valid, thus there is a need to incorporate a mechanism to detect when a user is not carrying the device such as [Hwang and Wohn 2013].

Fine-grain. Alternative and more advanced techniques have been proposed to achieve a more accurate result in estimating if people are in vicinity. These techniques are mainly based on ToA, TDoA, AoA and RSSI. Due to ease of implementation on mobile phones, most approaches focus on retrieving the Bluetooth/WiFi RSSI and then through a PLM, threshold-based classification or machine learning technique they try to estimate the interpersonal distance of the users.

An initial approach to estimate interpersonal distance through Bluetooth/WiFi RSSI, is the development of a PLM. The most simple method is Free space PLM that considers an ideal environment without reflections and obstacles. It requires a reference $R S S I_{r e f}$ measurement at a specific distance. Given the RSSI reference the model estimates the distance between the two devices. An improvement of this model is Office PLM [Wang et al. 2013] that modifies Free space PLM. In particular, it adds the impact of the indoor environment and especially of a normal office while assuming line-of-sight between the devices. Regarding the environmental parameter for indoor environments, there are predefined values for certain types of rooms that can be utilised. Alternatively, by retrieving RSSI samples at different distances and through an optimisation technique, researchers may compute their own parameters. Based on these generic PLMs, several variations have been proposed which add more parameters in order to consider other factors. One of these variations is BlueEye [Ghose et al. 2013] that strengthens the office PLM by incorporating two environmental constants and the relative orientation of the two devices; one of the factors which affects the RSSI is the directionality of device antennas. The output of the improved PLM was forwarded to k-means clustering to estimate users' interpersonal distance.

Stankovic [Li et al. 2013] applies a PLM with computed parameter for indoor and outdoor environments to detect when people are in vicinity; the interpersonal distance boundary utilised is $3 \mathrm{~m}$. Regarding the WiFi interface, Matic [Matic et al. 2012] created an artificial dataset through an indoor PLM by leveraging WiFi RSSI measurements at $1 \mathrm{~m}$ distance. Then, he trained a Naïve Bayes with a KDE classifier to detect if people were at a distance to socially interact. Features utilised to train the classifier were average and maximum values of a 10 -sample window. Finally, Comm2Sense [Carreras et al. 2012], followed the same process for training a classifier on 20-sample window that determines in which interaction zone people socialise.

Discussion. Researchers managed to achieve an improved accuracy in distance estimation. In order to achieve this, techniques such as RSSI, ToA, TDoA, AoA were utilised. For the implementation of some of these methods on smartphones, there are particular requirements such as firmware modification, multiple antennas etc. Thus research has focussed mainly on leveraging RSSI provided from the core API of the majority of COTS mobile devices. Based on RSSI, various PLMs have been proposed for environments such as free space, indoor and office, which require certain parameters for the specific environment. Even given the environmental parameters, RSSI is prone to antenna type and orientation, human body absorption, reflections and obstacles. Authors in [Banerjee et al. 2010] and [Matic et al. 2012] strive to tackle this through machine learning techniques. They incorporated uncertainty in distance measurements and utilised a 20 -sample window on which certain statistics were computed. However, they performed only small-scale experiments while viability, reliability and robustness of such a solution in the real-world environment is not proven. Additionally, the number of samples (window) required should be taken into consideration. As the number of samples increases, depending on the sampling frequency of the RF interface, the waiting time for an inference may increase. Also, when using a large window of samples (e.g. 20 samples [Carreras et al. 2012]), the data may be outdated leading to erroneous inference results.

5.5.2. Spatial Arrangement. Kendon [Kendon 1990] introduced F-Formation referring to the spatial formation created by the participants during a social interaction. In more detail, an F-Formation can include various configurations such as face-to-face, side-by-side, rectangular, circular, semi-circular and L-Formation ${ }^{8}$. So, depending on the formation that participants frame, different information about their social relationship is conveyed. This signifies the importance of a user's spatial arrangement. Researchers in Virtual Compass [Banerjee et al. 2010] by considering the interpersonal distance among users in vicinity, they created a virtual map through computing the

${ }^{8}$ In L-Formation users' torsos draw a right angle, similar to letter L. 
Euclidean distance of the users. [Matic et al. 2012] utilised off-the-shelf mobile phones to detect social interactions. Each participant carried the device on a static body position. While knowing the position of the mobile phone relative to a user's body, they used the orientation of the mobile phone in order to detect forward direction of torsos, hence to estimate users' spatial formations.

Discussion. Virtual Compass [Banerjee et al. 2010] calculated users' relative spatial arrangement. Due to the lack of users' facing directions and absolute locations they were not able to estimate the absolute spatial arrangement of the users. As the approach incorporated both WiFi and Bluetooth RSSI to perform the computations, unless an energy efficient mechanism is added, this work is not suitable for continuous sensing applications. Also, RSSI is highly dependent on the environment and prone to human body absorption. In contrary, [Matic et al. 2012] used the orientation sensor to keep track of the user's facing direction. However, the orientation sensor is based on a fusion mechanism of accelerometer and magnetometer that is affected by accelerometer bias and magnetic disturbance. A fusion mechanism that incorporates gyroscope with a drift compensation approach could prove to be a more reliable solution. Researchers in this work increased the intrusiveness of the system by limiting the smartphone's wearing position to the user's belt. A less restrictive approach regarding user's wearing position would improve the pervasiveness of the system.

5.5.3. A disclosure of Environmental and Spatial Cues. The environment and space in which a social interaction is taking place conveys information. A brief comparison of state-of-the-art techniques was presented to understand and provide quantification mechanisms to allow the extraction of these types of information.

Interpersonal distance estimation is an explored field with several proposed approaches. The classification of these works was based on the modality utilised to perform distance estimation. Sound-based distance estimation is the most recent approach where scientists have shown interesting results. BeepBeep [Peng et al. 2007] was able to tackle the device synchronisation problem required in ToA-based methods. Audible beacons constitute an issue which could be tackled through ultra-sound beacons, however they are still in an immature phase regarding mobile phones. In RF-based approaches, there is high dependence between the accuracy and system complexity required. Techniques such as ToA, TDoA and AoA are mainly contingent on external stationary or mobile hardware which introduces a certain level of intrusiveness and also mobility issues. RSSI is a popular solution for estimating distance but is highly dependent on the environment and is characterised by large fluctuations. Overall, these methods are prone to the environment and to human body absorption which both introduce a considerable amount of error. Preliminary results have shown that ultra-sound methods could achieve accurate distance estimation. However, to our knowledge there is no evaluation in unconstrained real-world environments.

Regarding spatial arrangement of the users, its importance has been indicated in psychology [Kendon 1990] however there are not considerable amount of works. Researchers focussed on detecting the relative spatial arrangement of the users. Furthermore, relative spatial arrangement induces error, as the absolute position is not known and through various parameters researchers focus on reducing the location uncertainty. To our knowledge there is no analytical work in order to quantify the error induced by this approach. Absolute positioning systems may reduce the error introduced by estimating relatively the spatial arrangement of the users. This could be achieved by inertial tracking systems that are build upon these types of information bearing in mind the requirement for energy efficiency due to continuous sensing.

\subsection{Device Usage}

The term behavioural cues mainly refers to non-verbal signals that are conveyed from a person during a social interaction. This constraint does not only refer to physical presence, but also to a social interaction in different physical places. For example, during an SMS text conversation people emit social signals such as response time, call frequency, punctuation, emoticons etc. These are all a small part of features that could be extracted from the usage of a mobile device.

SenseMs [Amin et al. 2005], was one of the first works that argued about non-verbal signals in SMS messaging. [Falaki et al. 2010] logged user's interaction with the device in order to understand the effect on the network and the energy consumption. The data utilised in this work could be forwarded to a human behaviour understanding mechanism to extract contextual information. [Bauer and Lukowicz 2012] monitored calling and SMS text behaviour on the mobile phone of a person and categorised it to different social groups. [Li et al. 2013] utilised GPS and Calendar to understand the context of a social interaction, while logging call records to list the interlocutors. Altshuler [Altshuler et al. 2013] introduced six categories of features based on user's patterns, that could be retrieved from a mobile phone: a) Internet usage, b) Calls, c) SMS messages, d) Phone applications, e) Alarm clock, f) Location. BeWell [Lane et al. 2012] also monitored smartphone usage such as device charging, screen lock, power off etc. Apart from the previous works describing the features that could be extracted from mobile phones, Olivier [Oliver 2010] created dataset from 17300 Blackberry devices in which he logged data representative of the user's interaction with the device. These datasets could prove to be a useful mean for predicting user's context.

Discussion. The most important advantage of these types of signals, is that they are collected from virtual sensors. This type of information is stored locally on the device while a person uses it and can be retrieved at user's discretion. Researchers can collect these types of data through the device's API or a sensing framework. Then, 


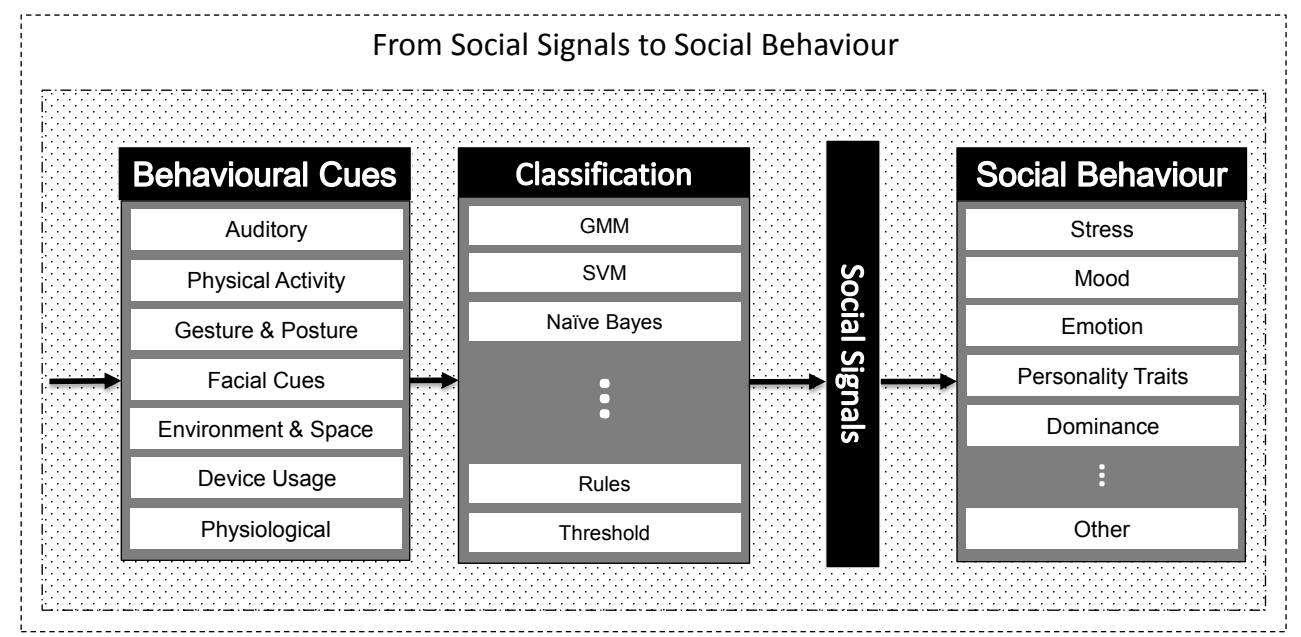

Fig. 5: Process of understanding social behaviour through social signals.

they can extract behavioural information with negligible energy consumption due to lack harnessing any of the burdensome physical sensors. These types of cues can be employed for long-term behavioural analysis of a user by inferring social characteristic patterns, but also to acquire contextual information.

\subsection{Physiological}

Extracting physiological characteristics of people during social interactions provides precious intelligence of natural state of the body. During a social interaction, based on the user's mental state, feelings, stress etc. the physiological body states are changing such as heart rate, skin temperature and humidity. For example people are interacting and due to the conversation context they feel stressed, which increases their heart rate and skin temperature. To detect these types of signals, researchers have focussed on Galvanic Skin Response (GSR), Respiratory Inductance Plethysmography (RIP), Electrocardiography (ECG), Electroencephalography (EEG) sensors.

AutoSense [Ertin et al. 2011] is a system composed of physiological sensors such as GSR, RIP, ECG, a mobile phone and a software component called FieldStream. Through external sensors (RIP and ECG), FieldStream performs a windowing pre-process, producing information such a window of R-peak locations, followed by feature extraction computing mean, variance, heart rate and respiration rate. NeuroPhone [Campbell et al. 2010] was the first work that incorporated mobile phones with a wireless electroencephalography (EEG) headset in order to perform actions on the mobile device emitted directly from a person's brain. The headset transmits data to the mobile phone, on which an initial averaging is performed followed by the appliance of a bandpass filter for noise reduction. Then, they utilise weighted classifiers, multivariate equal-prior Bayesian and decision stump classifiers. This approach could be applied in order to detect other brain signals which will lead to other social signal detection. imWell [Jovanov et al. 2013] connected a smartphone with a physiological sensor called Zephyr BioHarness 3 [Zephyr 2012] through Bluetooth interface. The mobile phone was monitoring and storing information about user's heart activity and later uploaded the data to a mHealth backend server for off-line processing. In [Gaggioli et al. 2012] authors employed physiological sensors in order to extract user's heart rate and variability. They deployed a feature extraction framework [Carbonaro et al. 2011] for filtering noise from ECG raw data in a robust and lightweight manner. SEPTIMU [Hong et al. 2012] utilised an earphone in which they incorporated a microphone in order to detect user's heart rate.

Discussion. Literature has mainly focussed on detecting a person's heart rate and skin temperature. This is performed through off-the-shelf sensors transmitting through wire(less) communication to mobile phones which conduct the inference. Off-the-shelf sensors have incorporated mechanisms of noise reduction, thus provide accurate estimations and usually do not need any pre-processing step. However, current approaches introduce a certain level of intrusiveness which should be considered during the design of Mobile SSP applications.

\section{FROM SOCIAL SIGNALS TO SOCIAL BEHAVIOUR INFERENCE}

Extraction of behavioural cues constitutes an abstraction layer, in which some preliminary knowledge is retrieved from raw sensor data. Combining these different types of information leads to the process of mining social signals. These signals convey significant information that characterise a person's feelings, mental state, interest and boredom during a social interaction. As the duration of the social signals is limited, a long-term analysis of the information they provide, will infer a person's social behaviour. In this section, we will outline different social behaviours that can be extracted from long-term analysis of certain social signals with respect to the behavioural cues analysed in the previous section (See Fig. 5). 


\subsection{Stress}

A social behaviour that has attracted a noticeable amount of interest among researchers is stress. Stress detection is mainly based on vocal, physical and physiological activity cues that are forwarded to a machine learning technique responsible for providing an estimation. As claimed by researchers, state-of-the-art techniques are able to achieve an acceptable accuracy over $80 \%$ in most cases.

AMMON [Chang et al. 2011] extracted prosodic features and utterances which were fed in a linear SVM and performed stress classification with $84.4 \%$ accuracy and $93.6 \%$ for stress increase-decrease. StressSense [Lu et al. 2012] exploited three different approaches to train two GMMs for stressed and neutral voice. They developed a universal model for all participants (71.3\% indoor accuracy), an adaptive model that starts from the universal model and through Maximum A Posteriori the model fits to a specific user (81.3\% supervised, 77.8\% unsupervised indoor accuracy), and finally a personalised model trained especially for each participant (82.9\% indoor accuracy). AutoSense [Ertin et al. 2011] requires physiological measures such as cardiovascular and respiratory data to infer about user's stress levels with $90 \%$ accuracy.

In [Gaggioli et al. 2012], authors combined activity, posture and physiological features through neural networks and a fuzzy logic algorithm in order to detect if a person is stressed. [Sun et al. 2012] performed stress classification in three different activities. Authors utilised physiological (ECG and GSR) and activities (e.g. sitting, walking, standing) features to determine if a person is stressed by applying J48 decision tree [Quinlan 1993], Bayesian Networks [Friedman et al. 1997] and an SVM [Cortes and Vapnik 1995] achieving the corresponding accuracies 92.4\%, 85\% and 84\%. [Muaremi et al. 2013] integrated physical activity, auditory, phone usage and heart rate variability features and achieved $61 \%$ accuracy for stress detection through multinomial logistic regression.

Discussion. As shown, existing literature has focussed on inferring stress through auditory, activity and physiological cues. AMMON [Chang et al. 2011] was able to manage $84.4 \%$ accuracy through prosody including glottal features and utterances given the trade-off of computational burden introduced by eigenvalues solving and other glottal features. In StressSense [Lu et al. 2012] as expected the personalised classifier achieved the highest accuracy. But for each user there is a need to train a separate model, followed by a supervised, an unsupervised adaptation model and last a generic classifier managing the worst accuracy. It is worth noting that external equipment was required in order to be able to perform speaker segmentation i.e an indoor array of microphones and outdoor a second smartphone.

AutoSense [Ertin et al. 2011] and [Gaggioli et al. 2012] require additional physiological equipment. This introduces a certain amount of intrusiveness but includes supplementary features such as heart-rate achieving multimodal inference. As opposed to AutoSense that utilises a J48 classifier which is prone to over-fitting, [Gaggioli et al. 2012] applies fuzzy logic-based rules that insert softer boundaries in the classification process. [Sun et al. 2012] with similar modalities achieved a relatively robust approach, without auditory cues, as for different types of classifiers there is a small variation in the overall claimed accuracy. Muaremi [Muaremi et al. 2013] utilised lightweight and easy to extract features but achieved the lowest accuracy for stress detection in the literature we reviewed.

In essence, the approaches for stress detection are concentrated either on auditory cues or on a combination of physiological, activity and auditory cues. Literature indicates that the most significant cues are auditory and physiological for detecting stress. In detail, researchers were able to detect stress accurately (over $80 \%$ ) by utilising only auditory data and extracting the aforementioned cues in contrast to physiological cues that were combined with additional modalities. Another important point that should be taken into consideration is the identification of the activity that the user is performing before executing the stress classification. Depending on the activity, the approach may be prone to false positives when carrying out intense activities. In conclusion, stress detection is a promising area and with the incorporation of the field of psychology will become mature, multi-modal and coherent.

\subsection{Emotion}

After analysing existing techniques for stress detection in Mobile SSP, in this subsection we focus on emotion detection. To detect emotion in a preliminary stage, researchers perform some simplification by focussing on the identification of major emotions such as happiness, anger, neutral, sadness etc. or just classifying if the user has positive or negative emotions. For emotion detection, scientists utilised audio datasets targeting different emotions and used them as training sets for machine learning techniques. Next we will present state-of-the-art techniques researchers utilised and provide a brief discussion about them.

At first, AMMON [Chang et al. 2011] extracted prosodic and spectral features from Belfast Naturalistic Database [Douglas-Cowie et al. 2003], and trained an SVM [Cortes and Vapnik 1995] classifier with 75\% accuracy for emotion recognition i.e. positive or negative. An important work is EmotionSense [Rachuri et al. 2010] which used Speech and Transcripts library [Liberman et al. 2002] to train an emotion recognition model and succeeded in $71 \%$ accuracy for 5 emotions based on prosodic features. Visage [Yang et al. 2013] detected users' emotion on mobile phones through facial expression detection [Belhumeur et al. 1997]. To evaluate their approach, they applied it on the JAFFE dataset [Lyons et al. 1998] achieving the corresponding accuracies: a) anger 82.16\%, b) disgust $79.68 \%$, c) fear $83.57 \%$, d) happiness $90.30 \%$, e) neutral $89.93 \%$, f) sadness $73.24 \%$ and g) surprise $87.52 \%$. 
In [Cho et al. 2009] they apply facial expression classification to detect a user's emotion and discriminate among four different emotions: a) neutral, b) joy, c) sad, d) surprise.

Discussion. As mentioned above, AMMON focussed only on extracting information regarding the users having a positive or negative emotion, which induces some generalisation. By performing classification with several feature sets, they achieved acceptable accuracy given the trade-off of computational load when including glottal timings in the feature set. Formant tracking including Newton-Raphson method is a high workload process, while in case the eigensolver fails additional burden is created by the construction of Toepliz matrices. FFT is another technique that is computationally expensive and should be considered before being applied on a mobile device intended for continuous inference.

EmotionSense includes components for adapting the sensing process based on the context. It showcases the effects in computation, communication cost and energy for performing the computations on the device or on a backend server. Authors trained the emotion detection model on a state-of-the-art library. However, there is a need to evaluate this model not only based on the trained library but also in a real-world environment to understand the robustness of the model. They performed speaker recognition on samples retrieved from 10 users. But there is no indication in what type of environment the data were collected from i.e. indoor, outdoor, with(out) ambient noise etc. Furthermore, adding Brownian noise is not sufficient to prove that the detection model is able to tolerate noise introduced by real-world environments. Similarly, the emotion recognition model was only evaluated on data from the training library. In essence, providing an evaluation of each of the components (speaker, emotion recognition) individually and as a holistic approach on real-world data, would indicate the robustness of the system in daily life monitoring. This necessitates the conduction of a larger-scale experiment for further analysis.

Visage utilises a well-established, robust and accurate method for face recognition combined with the device's orientation. However, this approach requires the user to hold the device in a position so as the mobile phone's camera is targeting the user's face. The face recognition approach through Fisherfaces [Belhumeur et al. 1997] provides tolerance in variations of lightning and expressions in comparison to other techniques such as Eigenfaces [Turk and Pentland 1991]. Also, it should be noted that the system operates in a supervised manner. Thus, it requires from the user to provide predefined facial expressions to construct a personalised model that classifies the seven distinct emotions.

In [Cho et al. 2009] authors were able to achieve a reasonable emotion recognition accuracy (70-80\%) for four emotions. They utilised a boosted Naïve Bayes for classification which introduces a certain computation load in the training process due to the creation of domain specific classifiers. Likewise, this approach is prone to the creation of domain specific classifiers for possible outliers, inducing over-fitting. The system requires pre-loaded images in the device and does not support real-time recognition of user's emotion through facial expressions.

Based on the above techniques certain parameters should be considered. The highest accuracy was achieved through facial expression recognition in Visage. However, it induces intrusiveness due to the requirement that the device's camera should target user's face. Also, the computational burden induced by face recognition and facial point tracking must be considered. EmotionSense managed an acceptable accuracy in an energy efficient manner, without requiring a specific on-body position of the device or any external hardware. AMMON provided only a preliminary classification result regarding the user's emotion but based on the application could be utilised. Regarding [Cho et al. 2009], the restricted inference context of the application indicates it as a less qualified system with respect to the others, for continuous sensing and inference.

\subsection{Mood}

In contrast to emotion, mood constitutes a generic emotional state difficult to describe and infer due to its multidimensionality. For that reason, researchers tried to approximate this emotional state through detecting several social signals based on their extraction complexity and significance with respect to mood. In order to detect the emitted social signals, researchers employed physiological sensors connected to mobile phones, on which they performed the inference. The most common social signals to infer mood in literature were arousal and valence while activeness and pleasure were also leveraged. Next, we will outline the techniques developed in literature and finally provide a brief comparison.

One of the first pieces of research, in which the authors interpreted user's mood was eMoto [Sundström et al. 2005]; through a sensor that was measuring pressure and arbitrary movement (gestures) the user was applying on it. They decoded valence, effort, pleasure and arousal. Another work of mood inference on mobile phones was [Gluhak et al. 2007]. Authors extracted physiological features from the user and through a certain threshold they were able to detect the level of arousal of the user i.e activated and relaxed. MoodScope [LiKamWa et al. 2013] is a mobile application that takes advantage of a user's phone usage patterns. Through a two-month training they were able to estimate mood i.e. activeness and pleasure, with $93 \%$ accuracy through multi-linear regression. Authors in eyeLook [Dickie et al. 2005] leveraged the ECS eye tracking tool to extract social signals such as attention though fixations and arousal through eye contact.

Discussion. The majority of works have concentrated on detecting mood, especially valence and arousal based on physiological features. Sensors measuring this type of features provide valuable information about a user's physiological state but require intrusive equipment that reduces the ubiquitous character of Mobile SSP. eMoto 
obliges the user to hold a stylus and does not consider the cultural background of the user e.g. people around Mediterranean sea tend to utilise many gestures during their conversation in comparison to people in Scandinavian that seldom perform gestures while discussing. [Gluhak et al. 2007] considered only the level of user's arousal, while the threshold-based classification approach is prone to misclassification when applied to people of different cultures.

As a continuation, MoodScope performed long-term analysis and included also phone usage data. The model's training required a considerable amount of time. Initial models had a poor performance (60-70\%) and only a personalised model was able to achieve high accuracy (93\%) in mood inference. Another disadvantage was that the mood detection model needed to be stored at a cloud infrastructure, requiring continuous internet communication and adding a noticeable burden on battery consumption. It is worth pointing out that the inference is based only on phone usage data, inducing minimal sensing energy consumption. eyeLook detected eye pupils and when they are dilated, which is considered as an indication of arousal. However, the eye-tracking mechanism is quite intrusive and is prone to false positives (eye detection) in an outdoor environment when it is sunny.

The emotional state of mood is not fully described by valence and arousal, indicating there is a requirement for incorporating other social signals to provide a more holistic approach. A missing parameter is the collaboration with psychologists, who will indicate the grammar of several modalities. This will provide the area with an understanding of the appropriate combination of social signals for inferring mood accurately given its multidimensionality.

\subsection{Personality Traits}

Following the emotional state of mood, a more static approach in terms of time is the characterisation of a person's personality traits [Goldberg 1993]. These are mainly parts of a person's character where a long-term analysis is required to identify them. Due to a broad spectrum of personality traits, the majority of researchers have focussed their works on the so called Big-Five in psychology: a) extraversion, b) emotional stability, c) conscientiousness, d) agreeableness and e) openness [Barrich and Mount 1991]. Although some works substituted emotional stability with neurotism, the overall concept of Big Five was the same.

Thus, in [Chittaranjan et al. 2011] authors monitored proximity among people and smartphone usage. For each trait a distinct set of features was fed into an SVM [Cortes and Vapnik 1995] and C4.5 [Quinlan 1993] classifiers to designate the Big-Five with accuracies in the scale 69-75.9\%. [Vinciarelli et al. 2012b] initially examined the correlation of auditory cues with personality traits and then showed that laughter and backchannel influence significantly increased the perception of social attractiveness. [Bogomolov et al. 2013] utilised data retrieved from mobile phone usage (calls, SMSs) and proximity (Bluetooth) to classify the happiness of the user with accuracy $80.81 \%$ through a Random Forest classifier.

Discussion. Given the afore analysed trade-off of proximity-based detection of social interactions (See Section 4) authors in [Chittaranjan et al. 2011] and [Bogomolov et al. 2013] utilised simple Bluetooth discovery in order to measure the social interactions in which a user participated. Although this method is easily implementable it introduces a noticeable amount of false positives that should be taken into account. A supplementary social interaction feature is the number of remote communications that existed among the users i.e. call and SMS logs. These features assume that the owner is the only user of the device and therefore there is a need to immunise it. Overall the achieved accuracy in both works is acceptable. However, there is a lack of incorporating several informative cues such auditory, activity-based etc., which in our opinion would provide a significant amount of information about the personality traits of the user.

In contrast, [Vinciarelli et al. 2012b] concentrates on auditory cues and shows the correlation between them and the Big-Five. However, they do not make any attempt to classify personality traits given these specific behavioural cues. The audio data are retrieved from recorded calls and do not include any data from real face-to-face situations. Furthermore, they extracted a large amount of features, some of them are computational demanding, rising issues regarding the applicability of such continuous feature extraction on mobile phones. Social attractiveness inference is based on laughter and backchannel which were proven reliable cues according to the authors. Other cues could provide additional information such as physical appearance, eye contact, mimicry in speech and movement etc.

In conclusion, inferring personality traits requires mining several social signals. The Big-Five is a first step for identifying the most important social signals related to one's personality. Nevertheless, literature includes works for distinct cues, thus an initiation of incorporating these different cues will gather a large amount of information and may provide a more holistic characterisation of a user's personality.

\subsection{Dominance}

After the analysis of inferring a user's personality traits, another characteristic of social behaviour is dominance. During a social interaction, a dominant person has higher social status in contrast to other submissive people. Dominance detection is a popular topic in SSP, which triggered the research on mobile devices as well. In Mobile SSP researchers mainly inferred dominance through auditory features by applying various distinct sets. In the following paragraphs existing literature of dominance inference on mobile phones will be described and analysed. 
We present [Aran and Gatica-Perez 2011], which is not based on mobile phones, but the methodology according to the authors is applicable to smartphones. In detail, they propose several approaches including simple rule-based inference. To introduce multi-modality, they perform feature-fusion based on the rank or a score and then utilise a rule-based classification. The features are extracted from audio (prosodic and turn-taking) and visual data. MeetingMediator [Kim et al. 2008] also detects dominant persons by computing turn-taking (speaking time, average speech segment length), prosodic features (variation in speech energy) and physical activity. A recent approach was introduced on SocioPhone [Lee et al. 2013b] in which they extracted prosodic and turn-taking features, and fed them in a supervised SVM [Cortes and Vapnik 1995].

Discussion. Regarding [Aran and Gatica-Perez 2011], the approach utilised in this work constitutes a lightweight and simple method, however it utilises only one feature. For that reason the authors decided to perform multimodal fusion. In detail, they applied fusion techniques based on rank or score to generate a unique feature that incorporates a series of multi-modal features. In MeetingMediator the only inference they perform is to compute the correlation of each person with respect to dominance, without developing a dominance detection model. Their conclusion about important features for dominance detection showcases high significance in the speaking time and speech energy variation. Thus indicates that a possible dominance detection model should include the aforementioned features. Finally, SocioPhone created an SVM-based dominance detection model but did not perform any evaluation to quantitatively understand the accuracy of the model. It should be noted that they were able to achieve high accuracy in the extraction of prosodic and turn-taking features in different environments and on-body positions of the device. Thus, a real-world evaluation would provide significant information about the applicability of such a model.

\subsection{Other social behaviours}

In previous subsections we analysed various social signals that contribute to the inference of some major social behaviour characteristics such as stress, emotion, mood, personality traits and dominance. Based on the literature, these are the main social characteristics that have driven researchers' interest. However, in parallel with social behaviours inference in these works, other social signals were mined which could trigger the interest of researchers to focus on other social behaviours or even invigorate existing inferences.

Other social behaviours were predicted in [Singh et al. 2013b], such as diversity (69\% accuracy), loyalty (69\% accuracy) and overspending (71\% accuracy) through phone usage information based on calls, SMSs and calendar. In [Berke et al. 2011] authors calculated the sociability of a person based on the time speaking during his participation in a conversation. In SocioPhone except from training a dominance detection model, they focussed on estimating characteristics such as interactivity through the number of turns-takings per minute, sparseness based on the number of silences with duration at least three seconds and skewness based on standard deviation of turn-takings.

Referring to a previous analysis about [Singh et al. 2013b], they were able to achieve a medium accuracy based on survey and receipt/credit data combined with proximity and phone usage data. The features were calculated on data collected over 1 year. Each social behaviour considered multiple modalities except overspending that utilised only proximity data fused with survey data. This method includes survey and receipts/credit data which induces human error. However, the integration of NFC technology allowing payments through mobile devices combined with incorporation of a connection of the system with user's bank account, will eliminate the human factor and create an opportunistic sensing system with higher accuracy.

[Berke et al. 2011] estimated sociability through auditory data in comparison to SociableSense [Rachuri et al. 2011] that combined speaking time with proximity data. The utilisation of multiple modalities allows the inference of a larger amount of information, such as co-location. In the case of an adaptive sensing system, proximity data can be utilised as a mean that triggers the conversation detection module. Thus, there is no need for continuous speech detection while avoiding missing events. In addition, other modalities could be incorporated for sociability inference such as calls, SMSs and instant messaging services. Finally, SocioPhone extracted with high accuracy prosodic and turn-taking features but similarly to dominance inference, they did not evaluate their models for interactivity, sparseness and skewness in real-world situations, in order to understand their applicability.

\section{APPLICATIONS}

In previous sections state-of-the-art techniques were analysed that may be used to infer social behaviour on mobile phones. Currently, we will showcase the leading application areas in which Mobile SSP can contribute or has already been utilised, indicating the importance and applicability of the field. Among a wide variety of applications where Mobile SSP can be leveraged, we have identified the main areas of health-care, organisational engineering and marketing.

\subsection{Health-care}

Health-care constitutes one of the most significant applications of Mobile SSP. A mobile device, through the large variety of internal and external sensors, allows constant monitoring of a patient in an unobtrusive way by simultaneously minimising the error introduced by human observer. They are able to detect minor and unnoticeable 
changes or anomalies in behaviour which may lead to diagnosing a disease even in the preliminary stages. Social behaviour-aware mobile devices, are capable of benefiting from the diagnosis and prevention of both physical and mental diseases [Pantelopoulos and Bourbakis 2010].

A notable amount of applications focus on the physical illness aspect of health-care, diagnosis, prevention and even prediction of various physical diseases. In detail, through continuous monitoring a minor behavioural variation that may not be noticeable to a human observer or even the patient himself, may be identified by anomaly detection in a patient's social behaviour. As an example [Seiter et al. 2013] observed the pain relief of a patient resulting from surgery, by detecting behavioural cues such as activity and posture. An application focussed more on prevention was presented in [Aharony et al. 2011], where a user's activity was inferred and combined with a reward system to engage users in a more healthy way of life. Also, there are situations where the patient requires long-term monitoring of physiological cues such as heart-rate, skin temperature etc. These may provide more detailed information about the overall health of the user and predicting common diseases such as obesity, high blood pressure and others including multiple sclerosis, Parkinson etc.

Apart from diagnosing physical diseases, Mobile SSP has applications in monitoring mental health as well. This area is described by changes and abnormalities outlined in patients' behaviour which can be identified through continuous monitoring of user behaviour. A common application of Mobile SSP is the quantification of user's stress levels in pursuance of limiting the effects of long-term high stress levels. This constitutes an application that requires short range monitoring. However, there are other mental diseases that require long-term monitoring. An example of this is the detection of evidence referring to the possibility of a person being depressed by collecting information such as mobility patterns, sociability etc.

Physical and mental diseases require a continuous, pervasive and ubiquitous monitoring tool that will provide significant information about anomalies or routines in a user's social behaviour. This will provide unbiased information to medical experts, enabling them to perform an initial diagnosis which will be verified by them.

\subsection{Organisational Engineering}

Another important application field is an automatic manner to quantify and analyse several aspects of organisational engineering i.e. employees' sociability, stress, job satisfaction [Das et al. 2010] including information flow, workload efficiency etc. These are all significant parameters that contribute to a healthier environment with respect to the employee and the organisation itself but also in increasing the efficiency and productivity of the organisation.

Mobile SSP will fabricate a new era in understanding, modelling and predicting the behaviour of organisations while introducing the importance of the social aspect. Social behaviour of an organisation's employees is an important parameter that is neglected today. So providing a quantification method for employees' sociability, stress levels etc. will indicate the job satisfaction employees are feeling, and accordingly perform the appropriate adjustments. As it has been shown in preliminary research [Olguín et al. 2009] it is achievable to comprehend the overall work-flow at an organisation by spotting lack of communication among different departments. This may lead to the identification of any existing or future eruption. Organisation are keen on being knowledgeable about the relationship among people, to reduce customer churn (e.g. churn prediction [Dasgupta et al. 2008] [Zhang et al. 2012]), to minimise any gap in the functional process of the corporation [Olguín et al. 2009] or to procure a suitable working team [Pentland 2012].

Organisations are dependent on their employees. This indicates the importance of being aware of their healthy social behaviour [Bateman and Organ 1983] to cope with early identified issues such as lack of intercommunication among various teams. Additionally, Mobile SSP may also identify possible unsatisfied customers and further assist in a correctly structured organisation. In conclusion, Mobile SSP has the potential to provide various enablers in the field of organisational engineering.

\subsection{Marketing}

Finally an area that several applications of Mobile SSP we foresee will emerge, is marketing. Social sciences have become essential in marketing, due to the comprehension of human behaviour required to fulfil the appropriate needs [Gardner 1985]. The knowledge of the user's general but also present social behaviour constitutes a new parameter in the area of marketing [Chen et al. 2009].

One of the benefits of Mobile SSP is the ability to provide a personalisation aspect in today's generalised marketing campaigns. This allows the identification of certain perspectives of user's behaviour. Following, it will enable marketers to target their campaigns to a specific audience that is keen on or open to the promotional target [Adomavicius and Tuzhilin 2011]. As an extension, modelling user's social behaviour through a mobile device may guide marketing to a new era, in which the environment will adjust automatically based on a user's predicted preference and mood [Strohbach and Martin 2011]. Another application that would provide benefits is the identification of potential customers [Gorgoglione et al. 2011]. An example proposed by Pentland in [Pentland 2007] was through leveraging only characteristics of voice, they were able to predict negotiation outcomes. This achievement would constitute an enabler for telephone-based marketers. In particular, they will recognise in short-term customers willing to accept an offer, reducing the time and effort spent for customers unwilling to be convinced. 
In overall, as the field of marketing is largely correlated with the area of psychology, there is a large amount of applications that may benefit from Mobile SSP, in order to improve and facilitate customer understanding and personalised marketing.

\section{DISCUSSION}

Mobile SSP is an important domain that has started to gain a great deal of interest due to its wide applicability. Not only psychology, but as presented there are several fields that will potentially benefit from the growth of this area. As described, research has not concluded in the terms and the taxonomy of SSP. Thus, researchers are need to agree and finalise in the terminology of the field so a concrete area is created. This will directly affect the development of Mobile SSP, while also enhancing the modelling of social signals. Having modelled social signals will provide a more clear understanding and classification of which behavioural cues can lead to certain social signals. By analysing these signals, an explicit guideline will tutor researchers in mining social behaviour in the long-term.

As shown in Section 3, there are numerous works released in order to provide the appropriate abstraction for retrieving information from mobile device sensors. Some frameworks have reached a certain maturity. This enables the utilisation of these tools in the design and development of mobile social behaviour applications without the need for handling low-level procedures required for sensing, processing, storing and retrieving information. The majority of sensing frameworks are built based on modern software design patterns to ensure robustness, security, extendibility but also openness. The latter two characteristics are highly correlated through the common ground of allowing third-parties to develop their own applications upon these frameworks but also contribute custom modules to extend and improve them. In addition, selecting a sensing framework is ostensibly a complicated process. But the designer should understand that concluding on a certain framework will only constitute a (less) significant enabler in the application and will not limit its capabilities. Last but not least, the intelligence that some frameworks provide regarding energy-efficiency may prove to be an additional succour.

Detecting social interactions through mobile devices is a topic that has drawn researchers' attention. Several approaches have been proposed by leveraging COTS mobile phones. Researchers have focussed on detecting social interactions by utilising a single or multiple modalities. Each method has its own advantages and disadvantages. Most works have performed simple discovery due to the pervasive and robust character of the approach, given a large amount of error. However, this method does not limit the user on a specific wearing position, with a very low design and development complexity. It is less sensitive to environmental factors in comparison to other approaches, because of the large spatial range it covers. The biggest disadvantage of the approach is the large amount of false positives it provides, especially in crowded places. Researchers tried to tackle this error through distance estimation based on Bluetooth, WiFi or audio signals. These approaches are highly dependent on the environment while human body absorption constitutes a significant obstacle. Voice and conversation detection have been incorporated in social interaction inference to increase accuracy. It should be noted that conversation detection constitutes a great challenge which requires tackling. It is also highly dependent on the environment and the on-body position of the device. Thus, depending on the accuracy required by the social interaction detection system, a less or more complex approach could be utilised while also considering the development effort for each methodology.

In the development of custom mobile devices, the designer decides about the components required based on the targeting application. For that reason, there is an advantage to selecting a robust and accurate solution (sensor) that will constitute the appropriate denouement. Although this approach may provide a reliable and robust solution, the designer must put a lot of effort in limiting its intrusiveness. To this point there is no robust and reliable off-the-shelf solution for detecting social interactions on mobile phones in real-world environments.

At the moment, research has focussed mainly in extracting behavioural cues because of immediacy among the device and the cue. This stage is based on the engineering part of Mobile SSP and does not necessarily require the collaboration with psychologists. Researchers have been mining several types of behavioural cues. Among them are the auditory for which, although a lot of research was conducted already from SSP, researchers applied various of these techniques on mobile devices. Although the majority of them were successfully adjusted to smartphones, there are some techniques that increase the computational burden and the energy consumption, thus this should be taken into consideration. Physical activity detection is also a topic that has gained researchers attention from the point of accelerometer incorporation on COTS mobile phones. As described, this is not a burdensome process and can be executed on mobile phones with high accuracy. Gesture recognition is an arguable cue, that to this point required either complex video processing or the user to hold the mobile phone in the hand. This raises questions regarding its real-world applicability. Posture detection is also mainly based on accelerometer data, with(out) external hardware, which can reliably be inferred. However the on-body position of the device should be included in the process. Facial cues extraction are based primarily on burdensome video processing and object identification. This may not be ideal for continuous sensing applications despite the psychological importance of the cues. Environmental cues provide a significant view of the behavioural cues regarding the context. A reliable and robust solution for detecting interpersonal distance and spatial arrangement from COTS mobile phones in realworld environments is still not available. The device usage based cues may not provide information about face-toface interaction, but it constitutes a lightweight and unobtrusive that can indicate reliable contextual knowledge. 
Physiological cues have been extracted through specific external sensors that limit the ubiquitous character of the area. Nevertheless the type of cues they detect convey information with high significance.

As literature indicated, as opposed to extracting behavioural cues, mining social signals and social behaviours on mobile phones is still immature. This occurs due to some important reasons that researchers need to take into consideration. Social signals and social behaviours include a noticeable amount of psychological knowledge. They require systematic collaboration with psychologists which will indicate behavioural cues postulated for mining a certain type of social behaviour. To our knowledge there has not been any tutorial providing a clear guideline of state-of-the-art techniques utilised in each of the steps detecting social signals. A tutorial will provide a definite understanding of the area and the methodology of mining social signals and social behaviour. A popular social behaviour is stress which can be detected robustly through auditory, physiological and physical activity cues. Emotion and mood detection was also mainly performed by auditory, facial and physiological cues with over $70 \%$ accuracy. Different personality traits were primarily detected by auditory, proximity and phone usage cues indicating the need for incorporating additional cues in the inference process. Finally, dominance and social role of a person was focussed on auditory cues as in SSP neglecting information such as spatial arrangement.

Overall, Mobile SSP is a multidisciplinary area that acquires a considerable amount of knowledge from adjacent fields, indicating the importance of active collaboration. These will drive researchers to incorporate multiple modalities in each of the inference stages. Each of these modalities introduce a certain level of error, intrusiveness, computational burden and energy consumption that should be considered, as the area targets mobile phones characterised by autonomy issues.

\section{CHALLENGES}

In previous section a discussion about the overall area of Mobile SSP and its main components was presented, identifying the key outcomes of the literature review. This research drove us to conclude, in our opinion, in some of the most significant challenges of the area that require tackling. These challenges constitute potential opportunities for research regarding the overall area of Mobile SSP which will provide a significant stride in the development and evolution of the area. In the following subsections each of the challenges is described, while in some cases initial steps are outlined in order to fill these gaps and to provide a further reference to the reader.

\subsection{Context Recognition}

Context is one of the most important factors in affective [Zeng et al. 2009] and context-aware computing [Chen and Kotz 2000] [Baldauf et al. 2007], anticipatory sensing [Pejovic and Musolesi 2013] and in Mobile SSP. As described earlier, SSP delves to interpret social behaviour, that requires detection of interactions among people, intertwined with the context in which it is taking place. Acquiring the knowledge of context in a more efficacious way of monitoring and understanding social behaviour, is looming. Due to the broad meaning of the term, one proposed solution for context recognition is to limit the scope of an application in order to focus on certain aspects of a specific context (e.g. monitor productivity in organisations [Olguín et al. 2009]). However, comprehending and construing context is a great challenge, which requires attentive and systematic research to depict a more holistic view. An example of context recognition is to detect accurately social interactions among people which will function as a significant enabler of social signal recognition through mobile phones. An important step to understand context, is to combine different modalities in a seamless manner to infer social behaviour.

\subsection{Multi-modal Fusion}

At this point in time, research has mainly been focussing on extracting various behavioural cues by utilising different modalities. A limited part of them has tried to infer social behaviour, either through individual or by combining a few behavioural cues in a simplistic manner. Due to their continuously increasing computational power, mobile devices allow incessant sensing of various modalities without compromising the user's experience. In order to infer accurately social behaviour, merging information from physical and virtual sensors is an indispensable need. Novel fusion techniques may be developed to perform this data amalgamation, precluding information redundancy, increasing the classification accuracy and mining contingent additional social signals. Targeting the incorporation of multiple modalities through novel fusion techniques, researchers must be able to model the area with help of psychology to understand which combination of modalities will lead in the identification of certain social behaviour.

\subsection{Interdisciplinary Area}

Mobile SSP is an area that requires coordination of different fields, in each of the stages for mining social behaviour. Starting from the sensing layer, experts in different modalities need to cooperate to leverage the most from every modality by providing appropriate pre-processing, fusion and post-processing mechanisms. These stages include expertise mainly from Electrical and Computer Engineering such as signal processing. A rife approach to extract behavioural cues and social signals is by utilising machine learning techniques. Understanding the type of modalities required to extract a certain form of social behaviour, indicates that the most important collaboration is between Engineering and Psychology [Hekler et al. 2013]. Psychologists have great experience in social behaviour and could provide the guideline on how to infer different aspects of human behaviour. This will supply them with 
an automatic and concise way to monitor and understand social behaviour. In addition, a common challenge among the areas is the issue of acquiring the knowledge of ground truth.

\subsection{Ground Truth}

Another important challenge in Mobile SSP is the fact of establishing ground truth opportunistically in real-world experiments. In state-of-the-art methods including Mobile SSP and SSP, scientists have acquired ground truth through human observer, camera recordings or user data labelling. As mentioned before, all three methods are time-consuming and prone to human error. Establishing ground truth by asking the user to label the data, induces subjectivity from the user's perspective and eliminates the opportunistic character that is a core idea of the field. It relies on the user's willingness to provide the experiment's baseline. Another approach adopted by researchers is to perform experiments in a small scale and controlled environment such as a room, in order to estimate the accuracy while understanding the method's limitations. Knowing the limitations of the approach and achieving an acceptable accuracy for a particular application, leads to a concrete solution. This enabler is then deployed in a large scale environment to extract a higher level knowledge of a population with the accuracy that was established in the initial experiments. Although, this method has been evaluated in a controlled environment and achieved a particular accuracy, scaling the approach will introduce new sources of error that may need to be tackled. An alternative approach that will be utilised potentially as Mobile SSP is evolving, is considering as ground truth the outcome of state-of-the-art techniques. However, this method limits an enabler's results to the state-of-the-art technique's accuracy. Thus providing a viable methodology for establishing ground truth in social sciences and especially Mobile SSP while preserving user's privacy, is an imperative need.

\subsection{Privacy}

Every application that is directly or indirectly related to humans, is also correlated to privacy. For that reason a very important trade-off to be made during the design and implementation of a Mobile SSP application, is usability against privacy [Avancha et al. 2012]. Regarding usability, in this context we consider the opportunistic and non-intrusive character of Mobile SSP. The target of a Mobile SSP application is to extract a certain type of behavioural information from the user. However, this target should be achieved with respect to the user's privacy. Some solutions have been proposed to minimise the impact on the user's privacy in crowd-sensing application, where the data are first anonymised and then retrieved from the device [Cornelius et al. 2008]. Privacy could be preserved by performing sensing and inference of social behaviour on the user's device. Thus, the collected data are not transmitted to a third party application while the user has the ability to delete unwanted or sensitive information. In some cases, on-line inference is not applicable due to device resource limitations. In that sense, the designer should introduce a privacy preserving mechanism that protects users' anonymity but also allows them to manage and expose only the desirable information in an energy efficient manner.

\subsection{Energy Efficiency}

Today's mobile devices have evolved significantly in terms of sensing and computation during the last decade. But a remaining issue that is challenging researchers in the field of Mobile SSP is battery consumption. To tackle this challenge, scientists may adopt alternative techniques to continuous sensing and inference. One promising approach is to apply adaptive mechanisms (e.g. reinforcement learning) in both sensing and inference regarding the context in which user/device is in. Another proposed solution is to perform the computations with subtlety either on the device or on a backend server, in an adjusted manner based on user's preference and the device's status. In order to allow devices to cope with the continuous computational and energetic demand, applications should be able to adapt based on user's context e.g. to apply a conservative policy in situations were user's social behaviour is insignificant. Regardless the existing solutions, we have identified that there is a great deal of research that has yet to be conducted and requires exploitation in each of the stages during the inference process.

\section{CONCLUSIONS}

After Pentland's introduction of Honest Signals [Pentland 2008], the research community focussed on modelling, analysing and synthesising human behaviour in an automatic manner. This interest was raised mainly due to the novel point of view introduced by incorporating the social, spontaneous and native aspect of human behaviour. Capturing this type of physical signals is a challenge, but mobile devices with the pervasive, ubiquitous and unobtrusive characteristics are a candidate solution. Mobile devices are a personalised tool, that is able through intelligent learning techniques to adopt to its user's preferences. Additionally, it eliminates the person detection process of SSP and thus provide more accurate results though less computational demanding processes. In our opinion, Mobile SSP is a promising area but requires a great deal of effort to overcome its main challenges. The scientific community has to finalise the core term-definition in order to establish a common ground. There have been noticeable works at lower layers of extracting social behaviour on mobile devices, e.g open source sensing and context recognition frameworks that provide an important abstraction enhancement. Currently, to our knowledge, there is no concrete framework for detecting and measuring social interactions on mobile phones in contrast to wearable devices that are able to accurately identify face-to-face interactions. Also, context recognition based 
works have to be leveraged and combined with the theoretical knowledge from the field of psychology. This will lead to modelling and analysing an additional sizeable amount of various behavioural cues in an energy efficient way. However, mining social signals and combining them to infer a user's social behaviour is still an area in which limited research has been conducted due to lack of coordination with the field of psychology. By tackling the challenges of Mobile SSP a new realm will emerge with applications in several fields and providing numerous benefits to areas such as health-care, organisational engineering and marketing.

\section{ACKNOWLEDGMENTS}

This work is supported by European Commission through the FP7-SOCIOTAL-609112 EU Projects and also by the joint EU and Ministry of Internal Affairs and Communication (MIC, Japan), Research and Innovation action iKaaS, under EU Grant No. 643262.

\section{REFERENCES}

G. Adomavicius and A. Tuzhilin. 2011. Context-Aware Recommender Systems. In Recommender Systems Handbook, F. Ricci, L. Rokach, B. Shapira, and P. B. Kantor (Eds.). Springer US, 217-253.

N. Aharony, W. Pan, C. Ip, I. Khayal, and A. Pentland. 2011. Social fMRI: Investigating and Shaping Social Mechanisms in the Real World. Pervasive Mob. Comput. 7, 6 (Dec. 2011), 643-659.

Y. Altshuler, M. Fire, N. Aharony, Z. Volkovich, Y. Elovici, and A. Pentland. 2013. Trade-Offs in Social and Behavioral Modeling in Mobile Networks. In Proceedings of the 6th International Conference on Social Computing, Behavioral-Cultural Modeling and Prediction (SBP'13). Springer-Verlag, Heidelberg, 412-423.

A. K. Amin, B. Kersten, O. A. Kulyk, E. Pelgrim, J. Wang, and P. Markopoulos. 2005. The SenseMS: Enriching the SMS Experience for Teens by Non-verbal Means. In Human-Computer Interaction - INTERACT 2005, MariaFrancesca Costabile and Fabio Patern (Eds.). Lecture Notes in Computer Science, Vol. 3585. Springer Heidelberg, 962-965.

Y. Anokwa, C. Hartung, W. Brunette, G. Borriello, and A. Lerer. 2009. Open Source Data Collection in the Developing World. Computer 42, 10 (Oct 2009), 97-99.

A. Antoniou, E. Theodoridis, I. Chatzigiannakis, and G. Mylonas. 2011. Monitoring physical space using mobile phones for inferring social and contextual interactions. In Sensors, 2011 IEEE. 1616-1619.

O. Aran and D. Gatica-Perez. 2011. Analysis of Group Conversations: Modeling Social Verticality. In Computer Analysis of Human Behavior, Albert Ali Salah and Theo Gevers (Eds.). Springer London, 293-322.

S. Avancha, A. Baxi, and D. Kotz. 2012. Privacy in Mobile Technology for Personal Healthcare. ACM Comput. Surv. 45, 1, Article 3 (Dec. 2012), 54 pages.

M. Azizyan, I. Constandache, and R. Roy Choudhury. 2009. SurroundSense: Mobile Phone Localization via Ambience Fingerprinting. In Proceedings of the 15th Annual International Conference on Mobile Computing and Networking (MobiCom '09). ACM, New York, NY, USA, 261-272.

M. Baldauf, S. Dustdar, and F. Rosenberg. 2007. A Survey on Context-Aware Systems. Int. J. Ad Hoc Ubiquitous Comput. 2, 4 (June 2007), 263-277.

N. Banerjee, S. Agarwal, P. Bahl, R. Chandra, A. Wolman, and M. Corner. 2010. Virtual Compass: Relative Positioning to Sense Mobile Social Interactions. In Proceedings of the 8th International Conference on Pervasive Computing (Pervasive'10). Springer-Verlag, Berlin, Heidelberg, $1-21$.

M. R. Barrich and M. K. Mount. 1991. The Big Five Personality Dimensions and Job Performance: A meta analysis. Personnel Psychology 44, 1 (1991), 1-26.

S. Basu. 2003. A linked-HMM model for robust voicing and speech detection. In Acoustics, Speech, and Signal Processing, 2003. Proceedings. (ICASSP '03). 2003 IEEE International Conference on, Vol. 1. I-816-I-819 vol.1.

T. S. Bateman and D. W. Organ. 1983. Job Satisfaction and the Good Soldier: The Relationship Between Affect and Employee Citizenship. Academy of Management Journal 26, 4 (1983), 587-595.

G. Bauer and P. Lukowicz. 2012. Can smartphones detect stress-related changes in the behaviour of individuals?. In Pervasive Computing and Communications Workshops (PERCOM Workshops), 2012 IEEE International Conference on. 423-426.

P. N. Belhumeur, J. P. Hespanha, and D. J. Kriegman. 1997. Eigenfaces vs. Fisherfaces: Recognition Using Class Specific Linear Projection. IEEE Trans. Pattern Anal. Mach. Intell. 19, 7 (July 1997), 711-720.

E. M. Berke, T. Choudhury, S. Ali, and M. Rabbi. 2011. Objective measurement of sociability and activity: mobile sensing in the community. Ann Fam Med 9, 4 (2011), 344-350.

A. Bogomolov, B. Lepri, and F. Pianesi. 2013. Happiness Recognition from Mobile Phone Data. In Social Computing (SocialCom), 2013 International Conference on. 790-795.

G. R. Bradski. 1998. Computer Vision Face Tracking For Use in a Perceptual User Interface. (1998).

P. M. Brunet, R. Cowie, D. Heylen, A. Nijholt, and M. Schrder. 2012. Conceptual frameworks for multimodal social signal processing. Journal on Multimodal User Interfaces 6, 3-4 (2012), 95-99.

A. Bulling, U. Blanke, and B. Schiele. 2014. A Tutorial on Human Activity Recognition Using Body-worn Inertial Sensors. ACM Comput. Surv. 46, 3, Article 33 (Jan. 2014), 33 pages.

A. Bulling, D. Roggen, and G. Tröster. 2011. What's in the Eyes for Context-Awareness? Pervasive Computing, IEEE 10, 2 (April 2011 ), $48-57$.

A. Campbell, T. Choudhury, S. Hu, H. Lu, M. K. Mukerjee, M. Rabbi, and R. D.S. Raizada. 2010. NeuroPhone: Brain-mobile Phone Interface Using a Wireless EEG Headset. In Proceedings of the Second ACM SIGCOMM Workshop on Networking, Systems, and Applications on Mobile Handhelds (MobiHeld '10). ACM, New York, NY, USA, 3-8.

N. Carbonaro, G. Anania, G. D. Mura, M. Tesconi, A. Tognetti, G. Zupone, and D. De Rossi. 2011. Wearable Biomonitoring System for Stress Management: A Preliminary Study on Robust ECG Signal Processing. In Proceedings of the 2011 IEEE International Symposium on a World of Wireless, Mobile and Multimedia Networks (WOWMOM '11). IEEE Computer Society, Washington, DC, USA, 1-6.

G. Cardone, A. Cirri, A. Corradi, L. Foschini, and D. Maio. 2013. MSF: An Efficient Mobile Phone Sensing Framework. International Journal of Distributed Sensor Networks 2013, 538937 (2013), 9. 
D. Carlson and A. Schrader. 2012. Dynamix: An open plug-and-play context framework for android. In Internet of Things (IOT), 2012 3rd International Conference on the. 151-158.

I. Carreras, A. Matic, P. Saar, and V. Osmani. 2012. Comm2Sense: Detecting proximity through smartphones. In Pervasive Computing and Communications Workshops (PERCOM Workshops), 2012 IEEE International Conference on. 253-258.

K. Chang, D. Fisher, J. Canny, and B. Hartmann. 2011. How's My Mood and Stress?: An Efficient Speech Analysis Library for Unobtrusive Monitoring on Mobile Phones. In Proceedings of the 6th International Conference on Body Area Networks (BodyNets '11). ICST (Institute for Computer Sciences, Social-Informatics and Telecommunications Engineering), ICST, Brussels, Belgium, Belgium, 71-77.

T. L. Chartrand and J. A. Bargh. 1999. The chameleon effect: The perception-behavior link and social interaction. Journal of Personality and Social Psychology 76, 6 (1999), 893-910.

G. Chen and D. Kotz. 2000. A Survey of Context-Aware Mobile Computing Research. Technical Report. Hanover, NH, USA.

Y. Chen, D. Pavlov, and J. F. Canny. 2009. Large-scale Behavioral Targeting. In Proceedings of the 15th ACM SIGKDD International Conference on Knowledge Discovery and Data Mining (KDD '09). ACM, New York, NY, USA, 209-218.

G. Chittaranjan, J. Blom, and D. Gatica-Perez. 2011. Who's Who with Big-Five: Analyzing and Classifying Personality Traits with Smartphones. In Proceedings of the 2011 15th Annual International Symposium on Wearable Computers (ISWC'11). IEEE Computer Society, Washington, DC, USA, 29-36.

S.Y. Cho, T.T. Teoh, and Y.Y Nguwi. 2009. Development of an Intelligent Facial Expression Recognizer for Mobile Applications. In New Advances in Intelligent Decision Technologies, Kazumi Nakamatsu, Gloria Phillips-Wren, LakhmiC. Jain, and RobertJ. Howlett (Eds.). Studies in Computational Intelligence, Vol. 199. Springer Berlin Heidelberg, 21-29.

K. Choi, K.A. Toh, and H. Byun. 2011. Realtime Training on Mobile Devices for Face Recognition Applications. Pattern Recogn. 44, 2 (Feb. 2011), $386-400$.

T. Choudhury and S. Basu. 2004. Modeling Conversational Dynamics as a Mixed-Memory Markov Process. In Advances in Neural Information Processing Systems 17, L.K. Saul, Y. Weiss, and L. Bottou (Eds.). 281-288.

T. Choudhury, G. Borriello, S. Consolvo, D. Haehnel, B. Harrison, B. Hemingway, J. Hightower, P. "Pedja” Klasnja, K. Koscher, A. LaMarca, J. A. Landay, L. LeGrand, J. Lester, A. Rahimi, A. Rea, and D. Wyatt. 2008. The Mobile Sensing Platform: An Embedded Activity Recognition System. IEEE Pervasive Computing 7, 2 (2008), 32-41.

T. Choudhury and A. Pentland. 2003. Sensing and Modeling Human Networks Using the Sociometer. In Proceedings of the 7th IEEE International Symposium on Wearable Computers (ISWC '03). IEEE Computer Society, Washington, DC, USA, 216.

C. Cornelius, A. Kapadia, D. Kotz, D. Peebles, M. Shin, and N. Triandopoulos. 2008. Anonysense: Privacy-aware People-centric Sensing. In Proceedings of the 6th International Conference on Mobile Systems, Applications, and Services (MobiSys '08). ACM, New York, NY, USA, 211-224.

C. Cortes and V. Vapnik. 1995. Support-vector networks. Machine Learning 20, 3 (1995), 273-297.

F. Dabek, R. Cox, F. Kaashoek, and R. Morris. 2004. Vivaldi: A Decentralized Network Coordinate System. SIGCOMM Comput. Commun. Rev. 34, 4 (Aug. 2004), 15-26.

T. Das, P. Mohan, V. N. Padmanabhan, R. Ramjee, and A. Sharma. 2010. PRISM: Platform for Remote Sensing Using Smartphones. In Proceedings of the 8th International Conference on Mobile Systems, Applications, and Services (MobiSys '10). ACM, New York, NY, USA, 63-76.

K. Dasgupta, R. Singh, B. Viswanathan, D. Chakraborty, S. Mukherjea, A. A. Nanavati, and A. Joshi. 2008. Social Ties and Their Relevance to Churn in Mobile Telecom Networks. In Proceedings of the 11th International Conference on Extending Database Technology: Advances in Database Technology (EDBT'08). ACM, New York, NY, USA, 668-677.

C. Dickie, R. Vertegaal, C. Sohn, and D. Cheng. 2005. eyeLook: Using Attention to Facilitate Mobile Media Consumption. In Proceedings of the 18th Annual ACM Symposium on User Interface Software and Technology (UIST '05). ACM, New York, NY, USA, $103-106$.

E. Douglas-Cowie, R. Cowie, and M. Schroeder. 2003. The Description of Naturally Occurring Emotional Speech. In Proceedings of 15th International Congress of Phonetic Sciences. 2877-2880.

C. Doukas and I. Maglogiannis. 2010. A Fast Mobile Face Recognition System for Android OS Based on Eigenfaces Decomposition. In Artificial Intelligence Applications and Innovations, Harris Papadopoulos, AndreasS. Andreou, and Max Bramer (Eds.). IFIP Advances in Information and Communication Technology, Vol. 339. Springer Berlin Heidelberg, 295-302.

H. Drewes, A. De Luca, and A. Schmidt. 2007. Eye-gaze Interaction for Mobile Phones. In Proceedings of the 4th International Conference on Mobile Technology, Applications, and Systems and the 1st International Symposium on Computer Human Interaction in Mobile Technology (Mobility '07). ACM, New York, NY, USA, 364-371.

N. Eagle and A. Pentland. 2005. Social Serendipity: Mobilizing Social Software. IEEE Pervasive Computing 4, 2 (April 2005 ), $28-34$.

C. Efstratiou, I. Leontiadis, M. Picone, K. K. Rachuri, C. Mascolo, and J. Crowcroft. 2012. Sense and Sensibility in a Pervasive World. In Proceedings of the 10th International Conference on Pervasive Computing (Pervasive'12). Springer-Verlag, Berlin, Heidelberg, $406-424$.

P. Ekman. 1993. Facial expression and emotion. American Psychologist 48, 4 (1993), 384-392.

E. Ertin, N. Stohs, S. Kumar, A. Raij, M. al'Absi, and S. Shah. 2011. AutoSense: Unobtrusively Wearable Sensor Suite for Inferring the Onset, Causality, and Consequences of Stress in the Field. In Proceedings of the 9th ACM Conference on Embedded Networked Sensor Systems (SenSys '11). ACM, New York, NY, USA, 274-287.

H. Falaki, R. Mahajan, and D. Estrin. 2011. SystemSens: A Tool for Monitoring Usage in Smartphone Research Deployments. In Proceedings of the Sixth International Workshop on MobiArch (MobiArch '11). ACM, New York, NY, USA, 25-30.

H. Falaki, R. Mahajan, S. Kandula, D. Lymberopoulos, R. Govindan, and D. Estrin. 2010. Diversity in Smartphone Usage. In Proceedings of the 8th International Conference on Mobile Systems, Applications, and Services (MobiSys '10). ACM, New York, NY, USA, $179-194$.

S. Feese, B. Arnrich, G. Troster, M. Burtscher, B. Meyer, and K. Jonas. 2013. CoenoFire: Monitoring Performance Indicators of Firefighters in Real-world Missions Using Smartphones. In Proceedings of the 2013 ACM International Joint Conference on Pervasive and Ubiquitous Computing (UbiComp '13). ACM, New York, NY, USA, 83-92.

V. Filonenko, C. Cullen, and J. Carswell. 2010. Investigating ultrasonic positioning on mobile phones. In Indoor Positioning and Indoor Navigation (IPIN), 2010 International Conference on. 1-8.

V. Filonenko, C. Cullen, and J. D. Carswell. 2013. Indoor Positioning for Smartphones Using Asynchronous Ultrasound Trilateration. ISPRS International Journal of Geo-Information 2, 3 (2013), 598-620.

Y. Freund and R. E. Schapire. 1997. A Decision-theoretic Generalization of On-line Learning and an Application to Boosting. J. Comput. Syst. Sci. 55, 1 (Aug. 1997), 119-139. 
N. Friedman, D. Geiger, and M. Goldszmidt. 1997. Bayesian Network Classifiers. Mach. Learn. 29, 2-3 (Nov. 1997), 131-163.

J. Froehlich, M. Y. Chen, S. Consolvo, B. Harrison, and J. A. Landay. 2007. MyExperience: A System for in Situ Tracing and Capturing of User Feedback on Mobile Phones. In Proceedings of the 5th International Conference on Mobile Systems, Applications and Services (MobiSys '07). ACM, New York, NY, USA, 57-70.

Funf. 2011. Open Sensing Framework. (2011). [online] http://www.funf.org.

A. Gaggioli, G. Pioggia, G. Tartarisco, G. Baldus, M. Ferro, P. Cipresso, S. Serino, A. Popleteev, S. Gabrielli, R. Maimone, and others. 2012. A System for Automatic Detection of Momentary Stress in Naturalistic Settings. Studies in Health Technology and Informatics 181 (2012), $182-186$.

M. P. Gardner. 1985. Mood States and Consumer Behavior: A Critical Review. Journal of Consumer Research 12, 3 (1985), pp. 281-300.

A. Ghose, C. Bhaumik, and T. Chakravarty. 2013. BlueEye: A System for Proximity Detection Using Bluetooth on Mobile Phones. In Proceedings of the 2013 ACM Conference on Pervasive and Ubiquitous Computing Adjunct Publication (UbiComp ' 13 Adjunct). ACM, New York, NY, USA, 1135-1142.

P. K. Ghosh, A. Tsiartas, and S. Narayanan. 2011. Robust Voice Activity Detection Using Long-Term Signal Variability. Audio, Speech, and Language Processing, IEEE Transactions on 19, 3 (March 2011), 600-613.

A. Gluhak, M. Presser, L. Zhu, S. Esfandiyari, and S. Kupschick. 2007. Towards Mood Based Mobile Services and Applications. In Proceedings of the 2Nd European Conference on Smart Sensing and Context (EuroSSC'07). Springer-Verlag, Berlin, Heidelberg, $159-174$.

E. Goffman. 1963. Behavior in Public Places: Notes on the Social Organization of Gatherings. Free Press.

L. R. Goldberg. 1993. The structure of phenotypic personality traits. American Psychologist 48, 1 (1993), 26-34.

Google Glass. 2013. Wearable Technology. (2013). [online] http://www.google.com/glass.

M. Gorgoglione, U. Panniello, and A. Tuzhilin. 2011. The Effect of Context-aware Recommendations on Customer Purchasing Behavior and Trust. In Proceedings of the Fifth ACM Conference on Recommender Systems (RecSys '11). ACM, New York, NY, USA, 85-92.

G. Groh, A. Lehmann, J. Reimers, M. R. Friess, and L. Schwarz. 2010. Detecting Social Situations from Interaction Geometry. In Proceedings of the 2010 IEEE Second International Conference on Social Computing (SOCIALCOM '10). IEEE Computer Society, Washington, DC, USA, $1-8$.

R. M. Groves. 2004. Survey errors and survey costs. Vol. 536. John Wiley \& Sons.

E. T. Hall. 1966. The hidden dimension. Doubleday.

M.A. Hall and G. Holmes. 2003. Benchmarking attribute selection techniques for discrete class data mining. Knowledge and Data Engineering, IEEE Transactions on 15, 6 (Nov 2003), 1437-1447.

E. B. Hekler, P. Klasnja, J. E. Froehlich, and M. P. Buman. 2013. Mind the Theoretical Gap: Interpreting, Using, and Developing Behavioral Theory in HCI Research. In Proceedings of the SIGCHI Conference on Human Factors in Computing Systems (CHI '13). ACM, New York, NY, USA, 3307-3316.

E. Hjelmas and B. K. Low. 2001. Face Detection: A Survey. Computer Vision and Image Understanding 83, 3 (2001), 236 - 274.

D. Hong, B. Zhang, Q. Li, S. Nirjon, R. Dickerson, G. Shen, X. Jiang, and J. Stankovic. 2012. SEPTIMU: Continuous In-situ Human Wellness Monitoring and Feedback Using Sensors Embedded in Earphones. In Proceedings of the 11th International Conference on Information Processing in Sensor Networks (IPSN '12). ACM, New York, NY, USA, 159-160.

S. A. Hoseinitabatabaei, A. Gluhak, and R. Tafazolli. 2013. A Survey on Smartphone-based Systems for Opportunistic User Context Recognition. ACM Comput. Surv. 45, 3, Article 27 (July 2013), 51 pages.

S. A. Hoseinitabatabaei, A. Gluhak, and R. Tafazolli. 2014. Towards a position and orientation independent approach for pervasive observation of user direction with mobile phones. Pervasive and Mobile Computing 0 (2014), -.

S. A. Hoseinitabatabaei, A. Gluhak, R. Tafazolli, and W. Headley. 2013. Design, Realization, and Evaluation of uDirect - An approach for Pervasive Observation of User Facing Direction on Mobile Phones. IEEE Transactions on Mobile Computing 99, PrePrints (2013), 1.

W. Hu, G. Cao, S. V. Krishanamurthy, and P. Mohapatra. 2013. Mobility-Assisted Energy-Aware User Contact Detection in Mobile Social Networks. 2013 IEEE 33rd International Conference on Distributed Computing Systems 0 (2013), 155-164.

G. Hua, T. Y. Yang, and S. Vasireddy. 2007. PEYE: Toward a Visual Motion Based Perceptual Interface for Mobile Devices. In Proceedings of the 2007 IEEE International Conference on Human-computer Interaction (HCI'07). Springer-Verlag, Berlin, Heidelberg, $39-48$.

S. Hwang and K. Wohn. 2013. VibroTactor: Low-cost Placement-aware Technique Using Vibration Echoes on Mobile Devices. In Proceedings of the Companion Publication of the 2013 International Conference on Intelligent User Interfaces Companion (IUI '13 Companion). ACM, New York, NY, USA, 73-74.

A. Iamnitchi, J. Blackburn, and N. Kourtellis. 2012. The Social Hourglass: An Infrastructure for Socially Aware Applications and Services. IEEE Internet Computing 16, 3 (May 2012), 13-23.

F. Ichikawa, J. Chipchase, and R. Grignani. 2005. Where's The Phone? A Study of Mobile Phone Location in Public Spaces. In Mobile Technology, Applications and Systems, 2005 2nd International Conference on. 1-8.

G. H. John and P. Langley. 1995. Estimating Continuous Distributions in Bayesian Classifiers. In Proceedings of the Eleventh Conference on Uncertainty in Artificial Intelligence (UAI'95). Morgan Kaufmann Publishers Inc., San Francisco, CA, USA, 338-345.

E. Jovanov, M. Milosevic, and A. Milenkovic. 2013. A mobile system for assessment of physiological response to posture transitions. In Engineering in Medicine and Biology Society (EMBC), 2013 35th Annual International Conference of the IEEE. 7205-7208.

H. Junker, O. Amft, P. Lukowicz, and G. Troster. 2008. Gesture spotting with body-worn inertial sensors to detect user activities. Pattern Recognition 41, 6 (2008), 2010-2024.

S. Kang, J. Lee, H. Jang, H. Lee, Y. Lee, S. Park, T. Park, and J. Song. 2008. SeeMon: Scalable and Energy-efficient Context Monitoring Framework for Sensor-rich Mobile Environments. In Proceedings of the 6th International Conference on Mobile Systems, Applications, and Services (MobiSys '08). ACM, New York, NY, USA, 267-280.

M. Keally, G. Zhou, G. Xing, J. Wu, and A. Pyles. 2011. PBN: Towards Practical Activity Recognition Using Smartphone-based Body Sensor Networks. In Proceedings of the 9th ACM Conference on Embedded Networked Sensor Systems (SenSys '11). ACM, New York, NY, USA, $246-259$.

A. Kendon. 1990. Conducting Interaction: Patterns of Behavior in Focused Encounters. Cambridge University Press.

E. J. Keogh, S. Chu, D. Hart, and M. J. Pazzani. 2001. An Online Algorithm for Segmenting Time Series. In Proceedings of the 2001 IEEE International Conference on Data Mining (ICDM '01). IEEE Computer Society, Washington, DC, USA, 289-296. 
T. Kim, A. Chang, L. Holland, and A. Pentland. 2008. Meeting Mediator: Enhancing Group Collaborationusing Sociometric Feedback. In Proceedings of the 2008 ACM Conference on Computer Supported Cooperative Work (CSCW '08). ACM, New York, NY, USA, $457-466$.

C. L. Kleinke. 1986. Gaze and eye contact: A research review. Psychological Bulletin 100, 1 (1986), 78-100.

A. Kotanen, M. Hannikainen, H. Leppakoski, and T.D. Hamalainen. 2003. Experiments on local positioning with Bluetooth. In Information Technology: Coding and Computing [Computers and Communications], 2003. Proceedings. ITCC 2003. International Conference on. 297303.

N. Koudenburg, T. Postmes, and E. H. Gordijn. 2011. Disrupting the flow: How brief silences in group conversations affect social needs. Journal of Experimental Social Psychology 47, 2 (2011), $512-515$.

J. Kukkonen, E. Lagerspetz, P. Nurmi, and M. Andersson. 2009. BeTelGeuse: A Platform for Gathering and Processing Situational Data. IEEE Pervasive Computing 8, 2 (April 2009), 49-56.

N. D. Lane, E. Miluzzo, H. Lu, D. Peebles, T. Choudhury, and A. T. Campbell. 2010. A Survey of Mobile Phone Sensing. Comm. Mag. 48, 9 (Sept. 2010), 140-150.

N. D. Lane, M. Mohammod, M. Lin, X. Yang, H. Lu, S. Ali, A. Doryab, E. Berke, T. Choudhury, and A. Campbell. 2012. BeWell: A Smartphone Application to Monitor, Model and Promote Wellbeing. In 5th International ICST Conference on Pervasive Computing Technologies for Healthcare. IEEE.

N. D. Lane, Y. Xu, H. Lu, S. Hu, T. Choudhury, A. T. Campbell, and F. Zhao. 2011. Enabling Large-scale Human Activity Inference on Smartphones Using Community Similarity Networks (Csn). In Proceedings of the 13th International Conference on Ubiquitous Computing (UbiComp '11). ACM, New York, NY, USA, 355-364.

H. Lee, Y. S. Choi, and S. Lee. 2012. Mobile Posture Monitoring System to Prevent Physical Health Risk of Smartphone Users. In Proceedings of the 2012 ACM Conference on Ubiquitous Computing (UbiComp '12). ACM, New York, NY, USA, 592-593.

H. Lee, Y. S. Choi, S. Lee, and E. Shim. 2013a. Smart Pose: Mobile Posture-aware System for Lowering Physical Health Risk of Smartphone Users. In CHI'13 Extended Abstracts on Human Factors in Computing Systems (CHI EA '13). ACM, New York, NY, USA, $2257-2266$.

Y. Lee, C. Min, C. Hwang, J. Lee, I. Hwang, Y. Ju, C. Yoo, M. Moon, U. Lee, and J. Song. 2013b. SocioPhone: Everyday Face-to-face Interaction Monitoring Platform Using Multi-phone Sensor Fusion. In Proceeding of the 11th Annual International Conference on Mobile Systems, Applications, and Services (MobiSys '13). ACM, New York, NY, USA, 375-388.

D. Li, D. Winfield, and D. J. Parkhurst. 2005. Starburst: A Hybrid Algorithm for Video-based Eye Tracking Combining Feature-based and Modelbased Approaches. In Proceedings of the 2005 IEEE Computer Society Conference on Computer Vision and Pattern Recognition (CVPR'05) Workshops - Volume 03 (CVPR '05). IEEE Computer Society, Washington, DC, USA, 79-.

K. A. Li, T. Y. Sohn, S. Huang, and W. G. Griswold. 2008. Peopletones: A System for the Detection and Notification of Buddy Proximity on Mobile Phones. In Proceedings of the 6th International Conference on Mobile Systems, Applications, and Services (MobiSys '08). ACM, New York, NY, USA, 160-173.

Q. Li, S. Chen, and J. A. Stankovic. 2013. Multi-modal in-person interaction monitoring using smartphone and on-body sensors. In Body Sensor Networks (BSN), 2013 IEEE International Conference on. 1-6.

M. Liberman, K. Davis, M. Grossman, N. Martey, and J. Bell. 2002. Emotional Prosody Speech and Transcripts.

R. LiKamWa, Y. Liu, N. D. Lane, and L. Zhong. 2013. MoodScope: Building a Mood Sensor from Smartphone Usage Patterns. In Proceeding of the 11th Annual International Conference on Mobile Systems, Applications, and Services (MobiSys '13). ACM, New York, NY, USA, 389-402.

J. Liu, C. Wang, J. Qiao, W. Wang, and Y. Zhang. 2012. A machine-to-machine application: Body posture recognition on smartphones for intelligent remote control. In Cloud Computing and Intelligent Systems (CCIS), 2012 IEEE 2nd International Conference on, Vol. 02. 884888 .

J. Liu, L. Zhong, J. Wickramasuriya, and V. Vasudevan. 2009. uWave: Accelerometer-based Personalized Gesture Recognition and Its Applications. Pervasive Mob. Comput. 5, 6 (dec 2009), 657-675.

K. Liu, X. Liu, and X. Li. 2013b. Guoguo: Enabling Fine-grained Indoor Localization via Smartphone. In Proceeding of the 11th Annual International Conference on Mobile Systems, Applications, and Services (MobiSys '13). ACM, New York, NY, USA, $235-248$.

K. Liu, X. Liu, L. Xie, and X. Li. 2013c. Towards accurate acoustic localization on a smartphone. In INFOCOM, 2013 Proceedings IEEE. $495-499$.

S. Liu, Y. Jiang, and A. Striegel. 2013a. Face-to-Face Proximity Estimation Using Bluetooth On Smartphones. IEEE Transactions on Mobile Computing 99, PrePrints (2013), 1.

H. Lu, A. J. B. Brush, B. Priyantha, A. K. Karlson, and J. Liu. 2011. SpeakerSense: Energy Efficient Unobtrusive Speaker Identification on Mobile Phones. In Proceedings of the 9th International Conference on Pervasive Computing (Pervasive'11). Springer-Verlag, Berlin, Heidelberg, 188-205.

H. Lu, D. Frauendorfer, M. Rabbi, M. S. Mast, G. T. Chittaranjan, A. T. Campbell, D. Gatica-Perez, and T. Choudhury. 2012. StressSense: Detecting Stress in Unconstrained Acoustic Environments Using Smartphones. In Proceedings of the 2012 ACM Conference on Ubiquitous Computing (UbiComp '12). ACM, New York, NY, USA, 351-360.

H. Lu, W. Pan, N. D. Lane, T. Choudhury, and A. T. Campbell. 2009. SoundSense: Scalable Sound Sensing for People-centric Applications on Mobile Phones. In Proceedings of the 7th International Conference on Mobile Systems, Applications, and Services (MobiSys '09). ACM, New York, NY, USA, 165-178.

H. Lu, J. Yang, Z. Liu, N. D. Lane, T. Choudhury, and A. T. Campbell. 2010. The Jigsaw Continuous Sensing Engine for Mobile Phone Applications. In Proceedings of the 8th ACM Conference on Embedded Networked Sensor Systems (SenSys '10). ACM, New York, NY, USA, 71-84.

M. Lyons, S. Akamatsu, M. Kamachi, and J. Gyoba. 1998. Coding Facial Expressions with Gabor Wavelets. In Proceedings of the 3rd. International Conference on Face \& Gesture Recognition (FG '98). IEEE Computer Society, Washington, DC, USA, 200-.

A. Madan and A. Pentland. 2006. VibeFones: Socially Aware Mobile Phones. In Wearable Computers, 2006 10th IEEE International Symposium on. 109-112.

A. Matic, V. Osmani, A. Maxhuni, and O. Mayora. 2012. Multi-modal mobile sensing of social interactions. In Pervasive Computing Technologies for Healthcare (PervasiveHealth), 2012 6th International Conference on. 105-114.

I. Matthews and S. Baker. 2004. Active Appearance Models Revisited. Int. J. Comput. Vision 60, 2 (Nov. 2004), $135-164$.

E. Miluzzo, C. T. Cornelius, A. Ramaswamy, T. Choudhury, Z. Liu, and A. T. Campbell. 2010. Darwin Phones: The Evolution of Sensing and Inference on Mobile Phones. In Proceedings of the 8th International Conference on Mobile Systems, Applications, and Services (MobiSys '10). ACM, New York, NY, USA, 5-20. 
E. Miluzzo, N. D. Lane, K. Fodor, R. Peterson, H. Lu, M. Musolesi, S. B. Eisenman, X. Zheng, and A. T. Campbell. 2008. Sensing Meets Mobile Social Networks: The Design, Implementation and Evaluation of the CenceMe Application. In Proceedings of the 6th ACM Conference on Embedded Network Sensor Systems (SenSys '08). ACM, New York, NY, USA, 337-350.

S. Mitra and T. Acharya. 2007. Gesture Recognition: A Survey. Systems, Man, and Cybernetics, Part C: Applications and Reviews, IEEE Transactions on 37, 3 (May 2007), 311-324.

A. Muaremi, B. Arnrich, and G. Trster. 2013. Towards Measuring Stress with Smartphones and Wearable Devices During Workday and Sleep. BioNanoScience 3, 2 (2013), 172-183.

Myo. 2013. Gesture Control Armband. (2013). [online] https://www.thalmic.com/en/myo/.

S. Nirjon, R. F. Dickerson, P. Asare, Q. Li, D. Hong, J. A. Stankovic, P. Hu, G. Shen, and X. Jiang. 2013. Auditeur: A Mobile-cloud Service Platform for Acoustic Event Detection on Smartphones. In Proceeding of the 11th Annual International Conference on Mobile Systems, Applications, and Services (MobiSys '13). ACM, New York, NY, USA, 403-416.

D. Olguín, B. N. Waber, T. Kim, A. Mohan, K. Ara, and A. Pentland. 2009. Sensible Organizations: Technology and Methodology for Automatically Measuring Organizational Behavior. Trans. Sys. Man Cyber. Part B 39, 1 (Feb. 2009), 43-55.

E. Oliver. 2010. The Challenges in Large-scale Smartphone User Studies. In Proceedings of the 2Nd ACM International Workshop on Hot Topics in Planet-scale Measurement (HotPlanet '10). ACM, New York, NY, USA, Article 5, 5 pages.

V. Osmani, I. Carreras, A. Matic, and P. Saar. 2014. An analysis of distance estimation to detect proximity in social interactions. Journal of Ambient Intelligence and Humanized Computing 5, 3 (2014), 297-306.

A. Pantelopoulos and N. G. Bourbakis. 2010. A Survey on Wearable Sensor-based Systems for Health Monitoring and Prognosis. Trans. Sys. Man Cyber Part C 40, 1 (Jan. 2010), 1-12.

T. Park, J. Lee, I. Hwang, C. Yoo, L. Nachman, and J. Song. 2011. E-Gesture: A Collaborative Architecture for Energy-efficient Gesture Recognition with Hand-worn Sensor and Mobile Devices. In Proceedings of the 9th ACM Conference on Embedded Networked Sensor Systems (SenSys '11). ACM, New York, NY, USA, 260-273.

V. Pejovic and M. Musolesi. 2013. Anticipatory Mobile Computing: A Survey of the State of the Art and Research Challenges. CoRR abs/1306.2356 (2013).

C. Peng, G. Shen, Y. Zhang, Y. Li, and K. Tan. 2007. BeepBeep: A High Accuracy Acoustic Ranging System Using COTS Mobile Devices. In Proceedings of the 5th International Conference on Embedded Networked Sensor Systems (SenSys '07). ACM, New York, NY, USA, 1-14.

A. Pentland. 2007. Social Signal Processing [Exploratory DSP]. Signal Processing Magazine, IEEE 24, 4 (July 2007), $108-111$.

A. Pentland. 2008. Honest Signals: How They Shape Our World. The MIT Press.

A. Pentland. 2012. The new science of building great teams. Harvard Business Review 90, 4 (2012), 60-69.

K. Peternel, M. Poganik, R. Tavar, and A. Kos. 2012. A Presence-Based Context-Aware Chronic Stress Recognition System. Sensors 12, 11 (2012), 15888-15906.

C. Pino and I. Kavasidis. 2012. Improving mobile device interaction by eye tracking analysis. In Computer Science and Information Systems (FedCSIS), 2012 Federated Conference on. 1199-1202.

I. Poggi, F. DErrico, and A. Vinciarelli. 2012. Social signals: from theory to applications. Cognitive Processing 13, 2 (2012), 389-396.

R. J. Quinlan. 1993. C4.5: Programs for Machine Learning. Morgan Kaufmann Publishers Inc., San Francisco, CA, USA.

M. R. Ra, B. Liu, T. La Porta, and R. Govindan. 2012. Medusa: A Programming Framework for Crowd-Sensing Applications. In Proceedings of the 10th International Conference on Mobile Systems, Applications, and Services (MobiSys'12).

K. K. Rachuri, C. Efstratiou, I. Leontiadis, C. Mascolo, and P. J. Rentfrow. 2013. METIS: Exploring mobile phone sensing offloading for efficiently supporting social sensing applications. 2013 IEEE International Conference on Pervasive Computing and Communications (PerCom) 0 (2013), 85-93.

K. K. Rachuri, C. Mascolo, M. Musolesi, and P. J. Rentfrow. 2011. SociableSense: Exploring the Trade-offs of Adaptive Sampling and Computation Offloading for Social Sensing. In Proceedings of the 17th Annual International Conference on Mobile Computing and Networking (MobiCom '11). ACM, New York, NY, USA, 73-84.

K. K. Rachuri, M. Musolesi, C. Mascolo, P. J. Rentfrow, C. Longworth, and A. Aucinas. 2010. EmotionSense: A Mobile Phones Based Adaptive Platform for Experimental Social Psychology Research. In Proceedings of the 12th ACM International Conference on Ubiquitous Computing (Ubicomp '10). ACM, New York, NY, USA, 281-290.

A. A. Razak and M. I. Z. Abidin. 2005. Comparison Between Fuzzy and NN Method for Speech Emotion Recognition. In Proceedings of the Third International Conference on Information Technology and Applications (ICITA'05) Volume 2 - Volume 02 (ICITA '05). IEEE Computer Society, Washington, DC, USA, 297-302.

J. Reason. 1990. Human Error. Cambridge University Press.

A. Sashima, Y. Inoue, T. Ikeda, T. Yamashita, and K. Kurumatani. 2008. CONSORTS-S: A mobile sensing platform for context-aware services. In Intelligent Sensors, Sensor Networks and Information Processing, 2008. ISSNIP 2008. International Conference on. 417-422.

J. Saunders. 1996. Real-time discrimination of broadcast speech/music. In Acoustics, Speech, and Signal Processing, 1996. ICASSP-96. Conference Proceedings., 1996 IEEE International Conference on, Vol. 2. 993-996 vol. 2.

E. A. Schegloff. 2000. Overlapping talk and the organization of turn-taking for conversation. Language in Society 29 (1 2000 ), 1-63. Issue 01.

M. Schrder. 2003. Experimental study of affect bursts. Speech Communication 40, 12 (2003), 99 - 116.

J. Seiter, S. Feese, B. Arnrich, G. Tröster, O. Amft, L. Macrea, and K. Maurer. 2013. Activity Monitoring in Daily Life As an Outcome Measure for Surgical Pain Relief Intervention Using Smartphones. In Proceedings of the 2013 International Symposium on Wearable Computers (ISWC '13). ACM, New York, NY, USA, 127-128.

E. Selkirk. 1995. Sentence prosody: Intonation, stress, and phrasing. (1995).

C. Shepard, A. Rahmati, C. Tossell, L. Zhong, and P. Kortum. 2011. LiveLab: Measuring Wireless Networks and Smartphone Users in the Field. SIGMETRICS Perform. Eval. Rev. 38, 3 (Jan. 2011), 15-20.

Y. Shi, Y. Shi, and J. Liu. 2011. A Rotation Based Method for Detecting On-body Positions of Mobile Devices. In Proceedings of the 13th International Conference on Ubiquitous Computing (UbiComp '11). ACM, New York, NY, USA, 559-560.

V. K. Singh, L. Freeman, B. Lepri, and A. Pentland. 2013a. Predicting Spending Behavior Using Socio-mobile Features. In Social Computing (SocialCom), 2013 International Conference on. 174-179. 
V. K. Singh, L. Freeman, B. Lepri, and A. Pentland. 2013b. Predicting Spending Behavior Using Socio-mobile Features. Social Computing / IEEE International Conference on Privacy, Security, Risk and Trust, 2010 IEEE International Conference on 0 (2013), $174-179$.

V. Srinivasan and T. Phan. 2012. An Accurate Two-tier Classifier for Efficient Duty-cycling of Smartphone Activity Recognition Systems. In Proceedings of the Third International Workshop on Sensing Applications on Mobile Phones (PhoneSense '12). ACM, New York, NY, USA, Article 11, 5 pages.

J. Staiano, B. Lepri, N. Aharony, F. Pianesi, N. Sebe, and A. Pentland. 2012. Friends Don'T Lie: Inferring Personality Traits from Social Network Structure. In Proceedings of the 2012 ACM Conference on Ubiquitous Computing (UbiComp '12). ACM, New York, NY, USA, 321-330.

J. Stehl, N. Voirin, A. Barrat, C. Cattuto, L. Isella, J. F. Pinton, M. Quaggiotto, W. Van den Broeck, C. Rgis, B. Lina, and P. Vanhems. 2011. High-Resolution Measurements of Face-to-Face Contact Patterns in a Primary School. PLOS ONE 6, 8 (08 2011$)$, e23176.

M. Strohbach and M. Martin. 2011. Toward a Platform for Pervasive Display Applications in Retail Environments. IEEE Pervasive Computing 10,2 (2011), 19-27.

F. T. Sun, C. Kuo, H. T. Cheng, S. Buthpitiya, P. Collins, and M. Griss. 2012. Activity-Aware Mental Stress Detection Using Physiological Sensors. In Mobile Computing, Applications, and Services, Martin Gris and Guang Yang (Eds.). Lecture Notes of the Institute for Computer Sciences, Social Informatics and Telecommunications Engineering, Vol. 76. Springer Berlin Heidelberg, 211-230.

P. Sundström, A. Ståhl, and K. Höök. 2005. eMoto: Affectively Involving Both Body and Mind. In CHI '05 Extended Abstracts on Human Factors in Computing Systems (CHI EA '05). ACM, New York, NY, USA, 2005-2008.

TagMobile. 2003. RFID Centre: Indoor RFID Tracking. (2003). [online] http://www.ubisense.net/en/index.html.

M. Turk and A. Pentland. 1991. Eigenfaces for Recognition. J. Cognitive Neuroscience 3, 1 (Jan. 1991), 71-86.

Ubisense. 2003. Location Intelligence solutions. (2003). [online] http://www.ubisense.net/en/index.html.

M. Uddin and T. Nadeem. 2013. RF-Beep: A light ranging scheme for smart devices. In Pervasive Computing and Communications (PerCom), 2013 IEEE International Conference on. 114-122.

R. Vertegaal, C. Dickie, C. Sohn, and M. Flickner. 2002. Designing Attentive Cell Phone Using Wearable Eyecontact Sensors. In CHI '02 Extended Abstracts on Human Factors in Computing Systems (CHI EA '02). ACM, New York, NY, USA, $646-647$.

R. Vertegaal, R. Slagter, G. Van Der Veer, and A. Nijholt. 2001. Eye Gaze Patterns in Conversations: There is More to Conversational Agents Than Meets the Eyes. In Proceedings of the SIGCHI Conference on Human Factors in Computing Systems (CHI'01). ACM, New York, NY, USA, 301-308.

A. Vinciarelli, R. Murray-Smith, and H. Bourlard. 2010. Mobile Social Signal Processing: Vision and Research Issues. In Proceedings of the 12th International Conference on Human Computer Interaction with Mobile Devices and Services (MobileHCI '10). ACM, New York, NY, USA, $513-516$.

A. Vinciarelli, M. Pantic, and H. Bourlard. 2009. Social Signal Processing: Survey of an Emerging Domain. Image Vision Comput. 27, 12 (Nov. 2009), 1743-1759.

A. Vinciarelli, M. Pantic, D. Heylen, C. Pelachaud, I. Poggi, F. D’Errico, and M. Schroeder. 2012a. Bridging the Gap between Social Animal and Unsocial Machine: A Survey of Social Signal Processing. IEEE Transactions on Affective Computing 3, 1 (2012), 69-87.

A. Vinciarelli, H. Salamin, A. Polychroniou, G. Mohammadi, and A. Origlia. 2012b. From Nonverbal Cues to Perception: Personality and Social Attractiveness. In Proceedings of the 2011 International Conference on Cognitive Behavioural Systems (COST'11). Springer-Verlag, Berlin, Heidelberg, 60-72.

P. Viola and M. J. Jones. 2004. Robust Real-Time Face Detection. Int. J. Comput. Vision 57, 2 (May 2004), $137-154$.

Y. Wang, J. Lin, M. Annavaram, Q. A. Jacobson, J. Hong, B. Krishnamachari, and N. Sadeh. 2009. A Framework of Energy Efficient Mobile Sensing for Automatic User State Recognition. In Proceedings of the 7th International Conference on Mobile Systems, Applications, and Services (MobiSys '09). ACM, New York, NY, USA, 179-192.

Y. Wang, X. Yang, Y. Zhao, Y. Liu, and L. Cuthbert. 2013. Bluetooth positioning using RSSI and triangulation methods. In Consumer Communications and Networking Conference (CCNC), 2013 IEEE. 837-842.

J. Wu, G. Pan, D. Zhang, G. Qi, and S. Li. 2009. Gesture Recognition with a 3-D Accelerometer. In Ubiquitous Intelligence and Computing, Daqing Zhang, Marius Portmann, Ah-Hwee Tan, and Jadwiga Indulska (Eds.). Lecture Notes in Computer Science, Vol. 5585. Springer Berlin Heidelberg, 25-38.

D. Wyatt, T. Choudhury, J. Bilmes, and J. A. Kitts. 2011. Inferring Colocation and Conversation Networks from Privacy-sensitive Audio with Implications for Computational Social Science. ACM Trans. Intell. Syst. Technol. 2, 1, Article 7 (Jan 2011), 41 pages.

B. Xu, R. Yu, G. Sun, and Z. Yang. 2011. Whistle: Synchronization-Free TDOA for Localization. In Distributed Computing Systems (ICDCS), 2011 31st International Conference on. 760-769.

Z. Yan, V. Subbaraju, D. Chakraborty, A. Misra, and K. Aberer. 2012. Energy-Efficient Continuous Activity Recognition on Mobile Phones: An Activity-Adaptive Approach. In Proceedings of the 2012 16th Annual International Symposium on Wearable Computers (ISWC) (ISWC'12). IEEE Computer Society, Washington, DC, USA, 17-24.

J. Yang. 2009. Toward Physical Activity Diary: Motion Recognition Using Simple Acceleration Features with Mobile Phones. In Proceedings of the 1st International Workshop on Interactive Multimedia for Consumer Electronics (IMCE '09). ACM, New York, NY, USA, 1-10.

X. Yang, C. W. You, H. Lu, M. Lin, N. D. Lane, and A. T. Campbell. 2013. Visage: A Face Interpretation Engine for Smartphone Applications. In Mobile Computing, Applications, and Services, David Uhler, Khanjan Mehta, and JenniferL. Wong (Eds.). Lecture Notes of the Institute for Computer Sciences, Social Informatics and Telecommunications Engineering, Vol. 110. Springer Berlin Heidelberg, $149-168$.

Z. Zeng, M. Pantic, G. I. Roisman, and T. S. Huang. 2009. A Survey of Affect Recognition Methods: Audio, Visual, and Spontaneous Expressions. Pattern Analysis and Machine Intelligence, IEEE Transactions on 31, 1 (Jan 2009), 39-58.

Zephyr. 2012. BioHarness 3, Wireless Professional Heart Rate Monitor and Physiological Monitor with Bluetooth. (2012). [online] http://www. zephyranywhere.com/products/bioharness-3/.

X Zhang, J. Zhu, S. Xu, and Y. Wan. 2012. Predicting Customer Churn Through Interpersonal Influence. Know.-Based Syst. 28 (April 2012), 97-104.

W. Y. Zhao, R. Chellappa, P. J. Phillips, and A. Rosenfeld. 2003. Face Recognition: A Literature Survey. ACM Comput. Surv. 35, 4 (Dec. 2003), 399-458. 


\title{
Online Appendix to:
}

\section{A Survey on Mobile Social Signal Processing}

\author{
NIKLAS PALAGHIAS, University of Surrey \\ SEYED AMIR HOSEINITABATABAEI, University of Surrey \\ MICHELE NATI, University of Surrey \\ ALEXANDER GLUHAK, Intel Labs Europe \\ KLAUS MOESSNER, University of Surrey
}

\section{A. SUMMARY OF MOBILE SSP LITERATURE}

This section summarises in a table, all works analysed in literature review of Mobile SSP. Table III presents each work and classifies its components into the different stages of social behaviour inference. The initial taxonomy includes the distinct social behaviour inference stages of sensing, social interaction detection, behavioural cues extraction and from social signals to social behaviour inference. In sensing column we outline the type of sensor data utilised by a particular work. Then, social interaction column describes the methodology developed by researchers in order to estimate ongoing social interactions. Following, the column of behavioural cues refers to the approach induced by researchers to extract behavioural cues. In order to clarify this process, the column is divided into extracted features, method developed to perform decision making, and classification target i.e. the result of inference. Similarly, the social behaviour column includes the research conducted by each work in terms of understanding social behaviour. As social behaviour inference is performed through a decision mechanism, this column is also divided into the extracted features, the decision method and the classification target. In overall, Table III constitutes a summary and brief categorisation into Mobile SSP inference stages, of the works discussed in the literature review. This article categorised related works based on the developed methodology. To enhance reader's understanding of the various methodologies, Table III provides an alternative view by outlining each work and the corresponding methods for every stage of social behaviour inference.

(c) YYYY ACM 0000-0000/YYYY/01-ARTA $\$ 15.00$

DOI : http://dx.doi.org/10.1145/0000000.0000000 
Table III: Literature on Mobile Social Signal Processing.

\begin{tabular}{|c|c|c|c|c|c|c|c|c|}
\hline \multirow{2}{*}{ System } & \multirow{2}{*}{ Sensing } & \multirow{3}{*}{$\begin{array}{l}\text { Social } \\
\text { Interaction } \\
\text { Infrared }\end{array}$} & \multirow{2}{*}{\multicolumn{3}{|c|}{ Behavioural Cues }} & \multicolumn{3}{|c|}{ Social Behaviour } \\
\hline & & & Features & & Target & Features & Method & Target \\
\hline $\begin{array}{l}\text { Sociometer [Ch } \\
\text { hury and } \\
\text { Pentland } \\
\text { 2003] [Olguín } \\
\text { et al. 2009] }\end{array}$ & oudilicrophone & & $\begin{array}{l}\text { non-initial max- } \\
\text { imum of the } \\
\text { autocorrela- } \\
\text { tion, number of } \\
\text { auto-correlation } \\
\text { peaks, normal- } \\
\text { ized spectral } \\
\text { entropy }\end{array}$ & 2-layer HMM & $\begin{array}{l}\text { Conversation, } \\
\text { Turn-taking }\end{array}$ & $\begin{array}{l}\text { conversation du- } \\
\text { ration, frequency, } \\
\text { ratio of interac- } \\
\text { tion, centrality } \\
\text { scores }\end{array}$ & $\begin{array}{l}\text { social network } \\
\text { structure }\end{array}$ & $\begin{array}{l}\text { influence, embed- } \\
\text { dedness in com- } \\
\text { munity }\end{array}$ \\
\hline $\begin{array}{l}\text { [Bauer and } \\
\text { Lukowicz } \\
\text { 2012] }\end{array}$ & $\begin{array}{l}\text { GPS, WiFi, } \\
\text { Bluetooth, } \\
\text { Calls, SMS }\end{array}$ & $\begin{array}{l}\text { Bluetooth } \\
\text { Discovery }\end{array}$ & & & & $\begin{array}{l}\text { number and time } \\
\text { at locations, so- } \\
\text { cial interactions, } \\
\text { calls, SMS }\end{array}$ & $\begin{array}{l}\text { deviation, stan- } \\
\text { dard deviation, } \\
\text { confidence inter- } \\
\text { val }\end{array}$ & Stress \\
\hline $\begin{array}{l}\text { NeuroPhone [C } \\
\text { bell et al. } \\
2010]\end{array}$ & $\operatorname{andy} b G$ & $\begin{array}{l}\text { Person iden- } \\
\text { tification } \\
\text { from images } \\
\text { through } \\
\text { P300 brain } \\
\text { signals }\end{array}$ & & & & & & \\
\hline $\begin{array}{l}\text { AutoSense [Ert } \\
\text { et al. 2011] }\end{array}$ & $\begin{array}{l}\text { nECG, RIP, } \\
\text { GSR, Skin } \\
\text { Thermome- } \\
\text { ter, Ambient } \\
\text { Temperature } \\
\text { Sensor, Ac- } \\
\text { celerometer }\end{array}$ & & $\begin{array}{l}\text { Performing win- } \\
\text { dowing on raw } \\
\text { data, preliminary } \\
\text { features (virtual } \\
\text { sensors) }\end{array}$ & Analysis of HRV & $\begin{array}{l}\text { Conversation, } \\
\text { Activity, Posture }\end{array}$ & $\begin{array}{l}\text { mean, variance, } \\
\text { heart rate, respi- } \\
\text { ration rate }\end{array}$ & & Stress $90 \%$ \\
\hline $\begin{array}{l}\text { [Gaggioli } \\
\text { et al. 2012] }\end{array}$ & $\begin{array}{l}\text { ECG, Ac- } \\
\text { celerometer, }\end{array}$ & & raw ECG data & $\begin{array}{l}\text { QRS } \quad \text { detec- } \\
\text { tion [Carbonaro } \\
\text { et al. 2011] }\end{array}$ & $\begin{array}{l}\text { motion activity, } \\
\text { posture, } \\
\text { rate }\end{array}$ & $\begin{array}{l}\text { activity, heart } \\
\text { rate }\end{array}$ & $\begin{array}{l}\text { Neural Network, } \\
\text { Fuzzy Logic algo- } \\
\text { rithms }\end{array}$ & Stress \\
\hline $\begin{array}{l}\text { SurroundSense } \\
\text { izyan et al. } \\
2009 \text { ] }\end{array}$ & $\begin{array}{l}\text { [AkiFi, Cam- } \\
\text { era, GSM, } \\
\text { Microphone, } \\
\text { Accelerome- } \\
\text { ter }\end{array}$ & & $\begin{array}{l}\text { Sound, Light, } \\
\text { Color, WiFi, } \\
\text { Accelerometer } \\
\text { fingerprints }\end{array}$ & $\begin{array}{l}\text { colour clustering, } \\
\text { light extraction, } \\
\text { feature selection }\end{array}$ & social context & & & \\
\hline $\begin{array}{l}\text { Virtual Com- } \\
\text { pass [Baner- } \\
\text { jee et al. } \\
2010]\end{array}$ & $\begin{array}{l}\text { Fusion Blue- } \\
\text { tooth and } \\
\text { WiFi }\end{array}$ & $\begin{array}{l}\text { Bluetooth } \\
\text { and WiFi } \\
\text { RSSI }\end{array}$ & $\begin{array}{l}\text { Combined PLM, } \\
\text { Modelling Uncer- } \\
\text { tainty }\end{array}$ & $\begin{array}{l}\text { Proximity, Rela- } \\
\text { tive Spatial Ar- } \\
\text { rangement }\end{array}$ & & & & \\
\hline $\begin{array}{l}\text { [Cho et al. } \\
2009]\end{array}$ & Camera & & Image & 2-Layer SVM & $\begin{array}{lc}\text { Fiducial } & \text { Points } \\
\text { (Eyes, } & \text { Nose, } \\
\text { Mouth) } & \end{array}$ & $\begin{array}{l}\text { Local Gator and } \\
\text { Gini Features }\end{array}$ & $\begin{array}{ll}\text { Boosting } \\
\text { Bayesian }\end{array}$ & $\begin{array}{l}\text { Emotion (neutral } \\
76.3 \% \text {, joy } 78.3 \% \text {, } \\
\text { sad } 74.7 \% \text {, sur- } \\
\text { prise } 78.7 \% \text { ) }\end{array}$ \\
\hline $\begin{array}{l}\text { MyExperience } \\
\text { et al. 2007] }\end{array}$ & $\begin{array}{l}\text { Froekdtabn, } \\
\text { Bluetooth, } \\
\text { User interac- } \\
\text { tion, Device } \\
\text { state }\end{array}$ & & $\begin{array}{l}\text { Device charging, } \\
\text { SMS, Cellular } \\
\text { info }\end{array}$ & Data aggregation & Social Context & & & \\
\hline $\begin{array}{l}P E Y E \text { [Hua } \\
\text { et al. 2007] }\end{array}$ & $\begin{array}{l}\text { Video record- } \\
\text { ing }\end{array}$ & & $\begin{array}{l}\text { partitioned im- } \\
\text { age in } 4 \text { equal } \\
\text { regions }\end{array}$ & $\begin{array}{l}\text { block matching } \\
\text { in } 16 \times 16 \text { image } \\
\text { through three } \\
\text { step search, four } \\
\text { step, diamond, } \\
\text { hexagon, and } \\
\text { the adaptive } \\
\text { multiple-mode } \\
\text { search }\end{array}$ & $\begin{array}{l}\text { Gesture recogni- } \\
\text { tion }\end{array}$ & & & \\
\hline $\begin{array}{l}\text { e- } \\
\text { Gesture [Park } \\
\text { et al. 2011] }\end{array}$ & $\begin{array}{l}\text { Accelerometer, } \\
\text { Gyroscope }\end{array}$ & & $\begin{array}{l}\text { raw, delta, inte- } \\
\text { gral data for each } \\
\text { axis }\end{array}$ & $\begin{array}{l}\text { HMM, Viterbi } \\
\text { algorithm maxi- } \\
\text { mum likelihood }\end{array}$ & $\begin{array}{l}\text { Gesture recogni- } \\
\text { tion ( } 94.6 \% \text {, in } \\
4 \text { different con- } \\
\text { texts) }\end{array}$ & & & \\
\hline
\end{tabular}




\begin{tabular}{|c|c|c|c|c|c|c|c|c|}
\hline \multirow{2}{*}{ System } & \multirow{2}{*}{ Sensing } & \multirow{2}{*}{$\begin{array}{l}\text { Social } \\
\text { Interaction }\end{array}$} & \multicolumn{3}{|c|}{ Behavioural Cues } & \multicolumn{3}{|c|}{ Social Behaviour } \\
\hline & & & Features & Method & Target & Features & Method & Target \\
\hline $\begin{array}{l}\text { [Junker et al. } \\
\text { 2008] }\end{array}$ & $\begin{array}{l}\text { Orientation } \\
\text { sensor }\end{array}$ & & Pitch and Roll & $\begin{array}{ll}\text { SWAB } & \text { [Keogh } \\
\text { et al. } & 2001], \\
\text { HMM } & \\
\end{array}$ & $\begin{array}{l}\text { Gesture recogni- } \\
\text { tion }\end{array}$ & & & \\
\hline $\begin{array}{l}\text { uWave [Liu } \\
\text { et al. 2009] }\end{array}$ & Accelerometer & & $\begin{array}{l}\text { Quantized ac- } \\
\text { celerometer data }\end{array}$ & $\begin{array}{ll}\text { Dynamic } & \text { time } \\
\text { warping } & \end{array}$ & $\begin{array}{ll}\text { Gesture } & \text { recogni- } \\
\text { tion (8 } & \text { distinct } \\
\text { gestures, } & 98.6 \% \\
\text { accuracy) } & \\
\end{array}$ & & & \\
\hline $\begin{array}{l}\text { Myo } \\
\text { 2013] }\end{array}$ & $\begin{array}{l}\text { Electromyograp } \\
\text { sensor }\end{array}$ & & $\begin{array}{l}\text { electrical activity } \\
\text { of skeletal mus- } \\
\text { cles }\end{array}$ & & $\begin{array}{l}\text { Gesture recogni- } \\
\text { tion }\end{array}$ & & & \\
\hline $\begin{array}{l}S E P T I M U \text { [Hon } \\
\text { et al. 2012] }\end{array}$ & $\begin{array}{l}\text { gAccelerometer, } \\
\text { Gyroscope, } \\
\text { Microphone } \\
\text { in earphones }\end{array}$ & & Raw data & & $\begin{array}{l}\text { Head Posture, } \\
\text { Physiological } \\
\text { (Heart rate) }\end{array}$ & & & \\
\hline $\begin{array}{l}\text { Smart } \\
\text { Pose [Lee } \\
\text { et al. 2013a] }\end{array}$ & $\begin{array}{l}\text { Accelerometer, } \\
\text { Orientation } \\
\text { Sensor, Cam- } \\
\text { era }\end{array}$ & & Raw data & 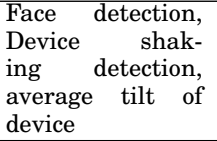 & $\begin{array}{l}\text { Head Posture } \\
\text { (User's neck tilt } \\
\text { angle) }\end{array}$ & & & \\
\hline $\begin{array}{l}\text { imWell [Jo- } \\
\text { vanov et al. } \\
2013]\end{array}$ & $\begin{array}{l}\text { Zephyr Bio- } \\
\text { Harness } 3\end{array}$ & & $\begin{array}{l}\text { Heart and Physi- } \\
\text { cal Activity }\end{array}$ & $\begin{array}{l}\text { Standard de- } \\
\text { viation and } \\
\text { Threshold-based }\end{array}$ & Body posture & & & \\
\hline $\begin{array}{l}\text { CONSORTS- } \\
S \quad \text { [Sashima } \\
\text { et al. 2008] }\end{array}$ & $\begin{array}{l}\text { Accelerometer, } \\
\text { Electrocar- } \\
\text { diograph, } \\
\text { Thermome- } \\
\text { ter }\end{array}$ & & $\begin{array}{l}\text { last } \\
\text { value(thermometer), } \\
\text { maximum value } \\
\text { (electrocar- } \\
\text { diograph), aver- } \\
\text { age(accelerometer), } \\
\text { variance and pri- } \\
\text { mary spectrum } \\
\text { frequency from } \\
\text { DFT }\end{array}$ & Decision Rules & $\begin{array}{l}\text { Body posture } \\
\text { (Standing, still, } \\
\text { facing down- } \\
\text { wards and up- } \\
\text { wards), Physical } \\
\text { Activity (stay- } \\
\text { ing, walking, } \\
\text { running), Phys- } \\
\text { iological (Heart } \\
\text { rate, skin tem- } \\
\text { perature) }\end{array}$ & & & \\
\hline 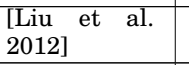 & \begin{tabular}{l|} 
Orientation \\
Sensor
\end{tabular} & & Pitch & Threshold & $\begin{array}{l}\text { Body posture (sit- } \\
\text { ting, standing) }\end{array}$ & & & \\
\hline $\begin{array}{l}\text { uDirect [Ho- } \\
\text { seinita- } \\
\text { batabaei } \\
\text { et al. 2013] }\end{array}$ & $\begin{array}{l}\text { Accelerometer, } \\
\text { Magnetome- } \\
\text { ter }\end{array}$ & & Raw data & $\begin{array}{lr}\text { Device } & \text { calibra- } \\
\text { tion, } & \text { Detect } \\
\text { relative } & \text { orien- } \\
\text { tation } & \text { between } \\
\text { user and device }\end{array}$ & $\begin{array}{l}\text { Body posture } \\
\text { (facing direction) }\end{array}$ & & & \\
\hline $\begin{array}{l}\text { SenseMs [Amin } \\
\text { et al. 2005] }\end{array}$ & $\begin{array}{l}\text { Virtual Sen- } \\
\text { sor (SMS) }\end{array}$ & & SMS & $\begin{array}{l}\text { Content Inter- } \\
\text { pretation (Facial } \\
\text { expressions, } \\
\text { avatars, colours, } \\
\text { size, location) }\end{array}$ & Phone Usage & & & \\
\hline $\begin{array}{l}\text { SeeMon [Kang } \\
\text { et al. 2008] }\end{array}$ & $\begin{array}{l}\text { BVP, GSR, } \\
\text { Light, Tem- } \\
\text { perature, } \\
\text { Humidity, } \\
\text { 2-axis ac- } \\
\text { celerometer, } \\
\text { GPS }\end{array}$ & & $\begin{array}{l}\text { Raw data, skin } \\
\text { conductance }\end{array}$ & & $\begin{array}{l}\text { Physical ac- } \\
\text { tivity (Strain, } \\
\text { movement), } \\
\text { Physiological } \\
\text { (Heart rate) }\end{array}$ & BVP & & Stress \\
\hline \multicolumn{9}{|c|}{\begin{tabular}{l|l} 
OpenDataKit [AnAlwwavailable \\
et al. 2009] & $\begin{array}{l}\text { physical } \\
\text { and virtual } \\
\text { sensors of } \\
\text { device }\end{array}$
\end{tabular}} \\
\hline $\begin{array}{l}\text { SystemSens [Fal } \\
\text { et al. 2011] }\end{array}$ & $\begin{array}{l}\text { aAdl available } \\
\text { physical } \\
\text { and virtual } \\
\text { sensors of } \\
\text { device }\end{array}$ & & & & & & & \\
\hline
\end{tabular}




\begin{tabular}{|c|c|c|c|c|c|c|c|c|}
\hline \multirow{2}{*}{ System } & \multirow{2}{*}{ Sensing } & \multirow{2}{*}{$\begin{array}{l}\text { Social } \\
\text { Interaction }\end{array}$} & \multicolumn{3}{|c|}{ Behavioural Cues } & \multicolumn{3}{|c|}{ Social Behaviour } \\
\hline & & & Features & Method & Target & Features & Method & Target \\
\hline $\begin{array}{l}\text { Funf [Funf } \\
\text { 2011] }\end{array}$ & $\begin{array}{l}\text { All available } \\
\text { physical } \\
\text { and virtual } \\
\text { sensors of } \\
\text { device }\end{array}$ & $\begin{array}{l}\text { Bluetooth } \\
\text { Discovery, } \\
\text { GSM, GPS }\end{array}$ & Accelerometer & Threshold & $\begin{array}{l}\text { Physical activity } \\
\text { (high, moderate, } \\
\text { low) }\end{array}$ & & & \\
\hline $\begin{array}{l}\text { Medusa [Ra } \\
\text { et al. 2012] }\end{array}$ & $\begin{array}{l}\text { All available } \\
\text { physical } \\
\text { and virtual } \\
\text { sensors of } \\
\text { device }\end{array}$ & & & & & & & \\
\hline $\begin{array}{l}\text { METIS [Rachur } \\
\text { et al. 2013] }\end{array}$ & $\begin{array}{l}\text { i Accelerometer, } \\
\text { Bluetooth, } \\
\text { microphone }\end{array}$ & $\begin{array}{l}\text { Bluetooth } \\
\text { discovery }\end{array}$ & $\begin{array}{l}\text { vibrations on } \\
\text { desks, location, } \\
\text { noise level, pres- } \\
\text { ence duration }\end{array}$ & & Social context & & & \\
\hline $\begin{array}{ll}M S F & \text { [Car- } \\
\text { done } & \text { et al. } \\
2013] & \end{array}$ & $\begin{array}{l}\text { All available } \\
\text { physical } \\
\text { and virtual } \\
\text { sensors of } \\
\text { device }\end{array}$ & & & & & & & \\
\hline $\begin{array}{l}\text { Anonysense [Cdr } \\
\text { nelius et al. } \\
2008]\end{array}$ & & & & & & & & \\
\hline $\begin{array}{l}\text { LiveLab [Shep- } \\
\text { ard et al. } \\
2011]\end{array}$ & $\begin{array}{l}\text { Phone usage, } \\
\text { Network us- } \\
\text { age }\end{array}$ & & & & & & & \\
\hline $\begin{array}{l}\text { PRISM [Das } \\
\text { et al. 2010] }\end{array}$ & $\begin{array}{l}\text { microphone, } \\
\text { camera, } \\
\text { GPS, ex- } \\
\text { ternal ac- } \\
\text { celerometer }\end{array}$ & GPS location & GPS, microphone & & Social context & & & \\
\hline $\begin{array}{l}\text { CenceMe [Miluze } \\
\text { et al. 2008] }\end{array}$ & $\begin{array}{l}\text { zoAccelerometer, } \\
\text { Microphone, } \\
\text { Camera, } \\
\text { GPS, Blue- } \\
\text { tooth }\end{array}$ & $\begin{array}{l}\text { Bluetooth } \\
\text { Discovery }\end{array}$ & $\begin{array}{l}\text { Accelerometer } \\
\text { (mean, std, peaks } \\
\text { number), Audio } \\
\text { (mean and std of } \\
\text { DFT), BLuetooth } \\
\text { MAC }\end{array}$ & $\begin{array}{l}\text { Decision tree, } \\
\text { Rule-based, }\end{array}$ & $\begin{array}{l}\text { Physical ac- } \\
\text { tivity (sitting, } \\
\text { standing, walk- } \\
\text { ing, running), } \\
\text { Auditory (Con- } \\
\text { versation), Social } \\
\text { context, }\end{array}$ & & & \\
\hline $\begin{array}{l}\text { BeTelGeuse [Kul } \\
\text { nen et al. } \\
2009]\end{array}$ & $\begin{array}{l}\text { kKntegrated } \\
\text { Sensors, } \\
\text { Camera, } \\
\text { GSM, Phone } \\
\text { Usage data, } \\
\text { GPS, Ac- } \\
\text { celeration, } \\
\text { Tempera- } \\
\text { ture, Heart } \\
\text { Rate }\end{array}$ & & $\begin{array}{l}\text { Accelerometer, } \\
\text { Heart Rate, } \\
\text { GSM, GPS }\end{array}$ & & $\begin{array}{l}\text { Physical Activity, } \\
\text { Social Context }\end{array}$ & & & \\
\hline $\begin{array}{l}\text { Jigsaw [Lu } \\
\text { et al. 2010] }\end{array}$ & $\begin{array}{l}\text { Accelerometer, } \\
\text { Microphone, } \\
\text { GPS }\end{array}$ & & $\begin{array}{lr}\begin{array}{l}\text { mean, } \\
\text { mean }\end{array} & \text { variance, } \\
\text { rate, } & \text { crossing } \\
\text { peak, sub-band } \\
\text { energy, sub-band } \\
\text { energy ratio, } \\
\text { spectral entropy, } \\
\text { MFCC r r }\end{array}$ & J48, GMM & $\begin{array}{l}\text { Physical Activity, } \\
\text { Posture (sit- } \\
\text { ting, standing), } \\
\text { Conversation }\end{array}$ & & & \\
\hline $\begin{array}{l}\text { EmotionSense }[\mathrm{F} \\
\text { et al. 2010] }\end{array}$ & $\begin{array}{l}\text { Radcleahirometer, } \\
\text { Bluetooth, } \\
\text { Location, } \\
\text { Microphone }\end{array}$ & $\begin{array}{l}\text { Bluetooth } \\
\text { Discovery, } \\
\text { Conversa- } \\
\text { tion Detec- } \\
\text { tion }\end{array}$ & $\begin{array}{ll}\text { Audio, } & \text { Ac- } \\
\text { celerometer } \\
\text { data }\end{array}$ & $\begin{array}{l}\text { HTK - GMM - } \\
\text { Maximum A Pos- } \\
\text { teriori }\end{array}$ & $\begin{array}{l}\text { Conversation } \\
\text { (Speaker Recog- } \\
\text { nition), Physical } \\
\text { Activity (move- } \\
\text { ment) }\end{array}$ & Speech segment & GMM - MAP & $\begin{array}{l}\text { Emotion (happi- } \\
\text { ness, sad, fear, } \\
\text { anger, neutral) }\end{array}$ \\
\hline
\end{tabular}




\begin{tabular}{|c|c|c|c|c|c|c|c|c|}
\hline \multirow{2}{*}{ System } & \multirow{2}{*}{ Sensing } & \multirow{2}{*}{$\begin{array}{l}\text { Social } \\
\text { Interaction }\end{array}$} & \multicolumn{3}{|c|}{ Behavioural Cues } & \multicolumn{3}{|c|}{ Social Behaviour } \\
\hline & & & Features & Method & Target & Features & Method & Target \\
\hline $\begin{array}{l}\text { SociableSense [1 } \\
\text { et al. 2011] }\end{array}$ & $\begin{array}{l}\text { Radcheherometer, } \\
\text { Bluetooth, } \\
\text { Location, } \\
\text { Microphone }\end{array}$ & $\begin{array}{l}\text { Bluetooth } \\
\text { Discovery, } \\
\text { Conversa- } \\
\text { tion Detec- } \\
\text { tion }\end{array}$ & $\begin{array}{ll}\text { Audio, } & \text { Ac- } \\
\text { celerometer } \\
\text { data }\end{array}$ & $\begin{array}{l}\text { HTK - GMM - } \\
\text { Maximum A Pos- } \\
\text { teriori }\end{array}$ & $\begin{array}{l}\text { Conversation } \\
\text { (Speaker Recog- } \\
\text { nition), Physical } \\
\text { Activity (move- } \\
\text { ment) }\end{array}$ & $\begin{array}{l}\text { Colocation, inter- } \\
\text { action patterns }\end{array}$ & $\begin{array}{l}\text { Compute relation } \\
\text { strength }\end{array}$ & Sociability \\
\hline $\begin{array}{l}\text { Ambient Dy- } \\
\text { namix [Carl- } \\
\text { son and } \\
\text { Schrader } \\
\text { 2012] }\end{array}$ & $\begin{array}{l}\text { All available } \\
\text { sensors of } \\
\text { device and } \\
\text { external }\end{array}$ & & $\begin{array}{l}\text { Accelerometer, } \\
\text { Zephyr Hx }\end{array}$ & & $\begin{array}{l}\text { Physical Activity } \\
\text { (Pedometer), } \\
\text { Physiological } \\
\text { (Heart Rate) }\end{array}$ & & & \\
\hline $\begin{array}{l}\text { Auditeur [Nir- } \\
\text { jon et al. } \\
2013 \text { ] }\end{array}$ & Microphone & & $\begin{array}{l}\text { FFT, ZCR, RMS, } \\
\text { 13-MFCCs, Low } \\
\text { Energy (Weak) } \\
\text { Frame rate } \\
\text { Spectral (En- } \\
\text { tropy, Energy, } \\
\text { Flux, Roll-off, } \\
\text { Centroid), Band- } \\
\text { width, Phase } \\
\begin{array}{l}\text { Deviation, Pitch, } \\
\text { and statistics of } \\
\text { these }\end{array}\end{array}$ & $\begin{array}{lr}\text { Naïve } & \text { Bayes, } \\
\text { Decision } & \text { Tree, } \\
\text { GMM, } & \text { MLP, } \\
\text { SVM, } & \text { kNN, } \\
\text { HMM } & \end{array}$ & $\begin{array}{ll}\text { Auditory } & \\
\text { (Prosody, } & \text { Turn- } \\
\text { taking, } & \text { Vocal } \\
\text { Outbursts, } & \text { Con- } \\
\text { versation) } & \end{array}$ & & & \\
\hline $\begin{array}{l}\text { Visage [Yang } \\
\text { et al. 2013] }\end{array}$ & Camera & & Shape, Texture & $\begin{array}{l}\text { Fisher Linear } \\
\text { Discriminant } \\
\text { Analysis (Fisher- } \\
\text { face) }\end{array}$ & $\begin{array}{l}\text { Facial cues (Fa- } \\
\text { cial Expression) }\end{array}$ & $\begin{array}{l}\text { Geometric, Ap- } \\
\text { pearance }\end{array}$ & SVM RBF kernel & $\begin{array}{l}\begin{array}{l}\text { Emotion } \\
\text { disgust, }\end{array} \text { (angry, } \\
\text { happy, neatral, } \\
\text { sad, surprice) }\end{array}$ \\
\hline $\begin{array}{l}\text { [Choi et al. } \\
\text { 2011] }\end{array}$ & Camera & & $\begin{array}{lr}\text { Local } & \text { face } \\
\text { tures } & \text { through } \\
\text { local } & \text { random } \\
\text { bases } & \\
\end{array}$ & $\begin{array}{l}\text { Sequential Neu- } \\
\text { ral Network }\end{array}$ & $\begin{array}{l}\text { Facial cues (Fa- } \\
\text { cial Expression) }\end{array}$ & & & \\
\hline $\begin{array}{l}\text { [Cho et al. } \\
\text { 2009] }\end{array}$ & Camera & & $\begin{array}{l}\text { Fiducial Points } \\
\text { with Local Gabor } \\
\text { and Gini }\end{array}$ & $\begin{array}{ll}\text { Boosting } & \text { Naïve } \\
\text { Bayesian } & \end{array}$ & $\begin{array}{l}\text { Facial cues (Fa- } \\
\text { cial Expression) }\end{array}$ & $\begin{array}{ll}\text { Facial } & \text { Expres- } \\
\text { sions } & \end{array}$ & & $\begin{array}{l}\text { Emotions } \text { (Neu- } \\
\text { tral } \\
\text { joy } 76.3 \%, 3 \% \text {, sad } \\
74.7 \%, \quad \text { surprise } \\
78.7 \%)\end{array}$ \\
\hline $\begin{array}{l}\text { TDoukas } \\
\text { and Ma- } \\
\text { glogiannis } \\
2010]\end{array}$ & Camera & & Image & $\begin{array}{l}\text { Eigenface de- } \\
\text { composition, Dis- } \\
\text { tance projection } \\
\text { and computation, } \\
\text { SVM }\end{array}$ & $\begin{array}{ll}\text { Facial Cues (Fa- } \\
\text { cial Points, Eye } \\
\text { Tracking) } & 96 \% \\
\text { accuracy } & \end{array}$ & & & \\
\hline $\begin{array}{l}\text { eyeLook [Dickie } \\
\text { et al. 2005] }\end{array}$ & $\begin{array}{l}\text { Camera, Eye } \\
\text { contact sen- } \\
\text { sors }\end{array}$ & $\begin{array}{l}\text { Eye contact } \\
\text { interaction } \\
\text { detection }\end{array}$ & $\begin{array}{l}\text { Eye contact sen- } \\
\text { sor readings }\end{array}$ & & $\begin{array}{l}\text { Facial cues (Eye } \\
\text { tracking - turn } \\
\text { taking) }\end{array}$ & & & \\
\hline $\begin{array}{l}\text { [Aharony } \\
\text { et al. 2011] }\end{array}$ & $\begin{array}{l}\text { WiFi, Blue- } \\
\text { tooth, cell } \\
\text { tower, } \\
\text { GPS, ac- } \\
\text { celerometer, } \\
\text { apps Info, } \\
\text { calls, SMS, } \\
\text { contacts, } \\
\text { phone/network } \\
\text { info, } \\
\text { power/screen } \\
\text { state, alarm } \\
\text { clock }\end{array}$ & $\begin{array}{l}\text { Bluetooth } \\
\text { discovery }\end{array}$ & $\begin{array}{l}\text { Accelerometer } \\
\text { data }\end{array}$ & $\begin{array}{l}\text { variance of mag- } \\
\text { nitude }\end{array}$ & Activity level & & & \\
\hline $\begin{array}{l}\text { [Altshuler } \\
\text { et al. 2013] }\end{array}$ & $\begin{array}{l}\text { Internet } \\
\text { usage, call, } \\
\text { SMS, apps, } \\
\text { alarm clock, } \\
\text { GPS, Blue- } \\
\text { tooth }\end{array}$ & $\begin{array}{l}\text { Bluetooth } \\
\text { discovery }\end{array}$ & & & & & & \\
\hline
\end{tabular}




\begin{tabular}{|c|c|c|c|c|c|c|c|c|}
\hline \multirow{2}{*}{ System } & \multirow{2}{*}{ Sensing } & \multirow{2}{*}{$\begin{array}{l}\text { Social } \\
\text { Interaction }\end{array}$} & \multirow{2}{*}{\multicolumn{3}{|c|}{ Behavioural Cues }} & \multicolumn{3}{|c|}{ Social Behaviour } \\
\hline & & & Features & & Target & Features & Method & Target \\
\hline $\begin{array}{l}\text { [Antoniou } \\
\text { et al. 2011] }\end{array}$ & Bluetooth & $\begin{array}{l}\text { Bluetooth } \\
\text { discovery } \\
\text { with static } \\
\text { and mobile } \\
\text { nodes }\end{array}$ & & & & & & \\
\hline $\begin{array}{l}\text { [Berke et al. } \\
\text { 2011] }\end{array}$ & $\begin{array}{l}\text { accelerometer, } \\
\text { microphone } \\
\text { barometric } \\
\text { pressure, } \\
\text { temperature, } \\
\text { humidity, } \\
\text { visible light, } \\
\text { infrared } \\
\text { light, battery }\end{array}$ & $\begin{array}{l}\text { Conversation } \\
\text { detection }\end{array}$ & $\begin{array}{l}\text { maximum auto- } \\
\text { correlation peak, } \\
\text { total number of } \\
\text { autocorrelation } \\
\text { peaks, and rel- } \\
\text { ative spectral } \\
\text { entropy of sound } \\
\text { detected }\end{array}$ & 2-layer HMM & $\begin{array}{l}\text { Conversation } \\
\text { (speech or not), } \\
\text { Activity level }\end{array}$ & $\begin{array}{l}\text { Conversation } \\
\text { time, question- } \\
\text { naire data (SF-36 } \\
\text { mental com- } \\
\text { ponent score } \\
\text { (MCS), CES-D) }\end{array}$ & & Well-being \\
\hline $\begin{array}{l}\text { [Bogomolov } \\
\text { et al. 2013] }\end{array}$ & $\begin{array}{l}\text { Calls, SMS, } \\
\text { Bluetooth }\end{array}$ & $\begin{array}{l}\text { Bluetooth } \\
\text { discovery }\end{array}$ & & & & $\begin{array}{l}\text { Device usage, } \\
\text { Proximity }\end{array}$ & $\begin{array}{l}\text { general phone us- } \\
\text { age, diversity, ac- } \\
\text { tive behaviours, } \\
\text { regularity }\end{array}$ & Happiness \\
\hline $\begin{array}{l}\text { [Bulling } \\
\text { et al. 2011] }\end{array}$ & $\begin{array}{l}\text { Accelerometer, } \\
\text { Bluetooth, } \\
\text { light sen- } \\
\text { sor, dry } \\
\text { electrodes }\end{array}$ & & face movement & $\begin{array}{l}\text { Wearable elec- } \\
\text { trooculography }\end{array}$ & $\begin{array}{l}\text { Facial cues (Eye } \\
\text { tracking) }\end{array}$ & & & \\
\hline $\begin{array}{l}\text { [Carbonaro } \\
\text { et al. 2011] }\end{array}$ & ECG & & & & & ECG data & $\begin{array}{l}\text { Kalman filter, } \\
\text { Pan Tompkins, R } \\
\text { Detection }\end{array}$ & Stress \\
\hline $\begin{array}{l}\text { Comm2Sense [0 } \\
\text { reras et al. } \\
2012]\end{array}$ & aWiFi & $\begin{array}{l}\text { Interpersonal } \\
\text { distance } \\
\text { based on } \\
\text { WiFi RSSI }\end{array}$ & $\begin{array}{l}\text { maximum and } \\
\text { mean of } 20- \\
\text { sample window } \\
\text { of WiFi RSSI }\end{array}$ & $\begin{array}{l}\text { Naïve Bayes with } \\
\text { KDE }\end{array}$ & $\begin{array}{l}\text { Interpersonal } \\
\text { Distance }\end{array}$ & & & \\
\hline $\begin{array}{l}A M M O N \text { [Chan } \\
\text { et al. } 2011]\end{array}$ & $\begin{array}{l}\text { lGPS, Calen- } \\
\text { dar, Micro- } \\
\text { phone }\end{array}$ & & & & & $\begin{array}{l}\text { mean, standard } \\
\text { deviation, kur- } \\
\text { tosis, skewness, } \\
\text { minimum, max- } \\
\text { imum range, } \\
\text { relative position, } \\
\text { ZCR, RMS en- } \\
\text { ergy, F0, HNR, } \\
\text { MFCC 1-12, } \\
\text { Glottal timings }\end{array}$ & linear SVM & Stress, Affect \\
\hline $\begin{array}{l}\text { [Chittaranjan } \\
\text { et al. 2011] }\end{array}$ & $\begin{array}{l}\text { Application } \\
\text { usage, Blue- } \\
\text { tooth, SMS, } \\
\text { calls }\end{array}$ & & $\begin{array}{l}\text { Bluetooth discov- } \\
\text { ery }\end{array}$ & $\begin{array}{l}\text { number of oc- } \\
\text { currences, mean, } \\
\text { median }\end{array}$ & & & & $\begin{array}{l}\text { Extraversion, } \\
\text { Agreeableness, } \\
\text { Conscientious- } \\
\text { ness, Emotional } \\
\text { Stability, Open- } \\
\text { ness to Experi- } \\
\text { ence }\end{array}$ \\
\hline 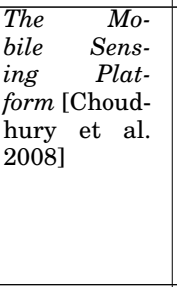 & $\begin{array}{l}\text { microphone, } \\
\text { light pho- } \\
\text { transistor, } \\
\text { accelerome- } \\
\text { ter, barom- } \\
\text { eter, ther- } \\
\text { mometer, IR, } \\
\text { humidity, } \\
\text { compass }\end{array}$ & & $\begin{array}{l}\text { linear and log- } \\
\text { scale frequency } \\
\text { coefficients, cep- } \\
\text { stral coefficients, } \\
\text { spectral entropy, } \\
\text { band-pass fil- } \\
\text { ter coefficients, } \\
\text { correlations, in- } \\
\text { tegrals, means, } \\
\text { variances }\end{array}$ & HMM, CRF & $\begin{array}{l}\text { Activity Recogni- } \\
\text { tion }\end{array}$ & & & \\
\hline $\begin{array}{l}\text { Social } \\
\text { Serendip- } \\
\text { ity [Eagle } \\
\text { and Pent- } \\
\text { land 2005] }\end{array}$ & Bluetooth & $\begin{array}{l}\text { Bluetooth } \\
\text { discovery }\end{array}$ & & & & & & \\
\hline
\end{tabular}




\begin{tabular}{|c|c|c|c|c|c|c|c|c|}
\hline \multirow{2}{*}{ System } & \multirow{2}{*}{ Sensing } & \multirow{2}{*}{$\begin{array}{l}\text { Social } \\
\text { Interaction }\end{array}$} & \multicolumn{3}{|c|}{ Behavioural Cues } & \multicolumn{3}{|c|}{ Social Behaviour } \\
\hline & & & Features & Method & Target & Features & Method & Target \\
\hline $\begin{array}{l}\text { [Drewes } \\
\text { et al. 2007] }\end{array}$ & Camera & & Video recording & ERICA & $\begin{array}{l}\text { Facial cues (Eye- } \\
\text { tracking) }\end{array}$ & & & \\
\hline $\begin{array}{l}\text { [Efstratiou } \\
\text { et al. 2012] }\end{array}$ & $\begin{array}{l}\text { Static and } \\
\text { mobile Blue- } \\
\text { tooth nodes }\end{array}$ & $\begin{array}{l}\text { Bluetooth } \\
\text { discovery }\end{array}$ & & & Social Context & & & \\
\hline $\begin{array}{l}\text { Coenofire [Feese } \\
\text { et al. 2013] }\end{array}$ & $\begin{array}{l}\text { Microphone, } \\
\text { Accelerome- } \\
\text { ter, Barom- } \\
\text { eter, Blue- } \\
\text { tooth }\end{array}$ & $\begin{array}{l}\text { Bluetooth } \\
\text { discovery }\end{array}$ & $\begin{array}{l}\text { Microphone, } \\
\text { accelerometer, } \\
\text { pressure data }\end{array}$ & $\begin{array}{l}\text { standard de- } \\
\text { viation of the } \\
\text { acceleration mag- } \\
\text { nitude, median of } \\
\text { the absolute lin- } \\
\text { ear acceleration } \\
\text { magnitude, inter- } \\
\text { quartile-range of } \\
\text { the absolute lin- } \\
\text { ear acceleration } \\
\text { magnitude, long- } \\
\text { term-spectral- } \\
\text { variability }\end{array}$ & $\begin{array}{l}\text { movement activ- } \\
\text { ity, movement } \\
\text { intensity, move- } \\
\text { ment variability, } \\
\text { speech activity }\end{array}$ & & & \\
\hline \begin{tabular}{ll}
\multicolumn{3}{l|}{ [Filonenko } \\
et & al. \\
$2010]$ & [Filo- \\
nenko et al. \\
2013 ]
\end{tabular} & $\begin{array}{l}\text { Microphone, } \\
\text { speakers, ac- } \\
\text { celerometer, } \\
\text { magnetome- } \\
\text { ter }\end{array}$ & & $\begin{array}{l}\text { Microphone data, } \\
\text { accelerometer, } \\
\text { magnetometer }\end{array}$ & $\begin{array}{l}\text { DSP filters, } \\
\text { trilateration, } \\
\text { ultrasound fre- } \\
\text { quencies }\end{array}$ & $\begin{array}{l}\text { Interpersonal } \\
\text { distance }\end{array}$ & & & \\
\hline $\begin{array}{l}\text { BlueEye [Ghose } \\
\text { et al. 2013] }\end{array}$ & Bluetooth & & Bluetooth RSSI & $\begin{array}{l}\text { Modified PLM } \\
\text { incl. devices' rel- } \\
\text { ative orientation }\end{array}$ & $\begin{array}{l}\text { Interpersonal } \\
\text { distance (proxim- } \\
\text { ity) }\end{array}$ & & & \\
\hline $\begin{array}{l}\text { [Gluhak } \\
\text { et al. 2007] }\end{array}$ & $\begin{array}{l}\text { Biomonitoring } \\
\text { sensors }\end{array}$ & & $\begin{array}{l}\text { avg heart beats } \\
\text { per min, root } \\
\text { mean square of } \\
\text { std, \% of differ- } \\
\text { ences between } \\
\text { adjacent beat-to- } \\
\text { beat intervals, } \\
\text { avg breaths per } \\
\text { min, EDA turn- } \\
\text { ing points, EDA } \\
\text { percentage of } \\
\text { increase, EDA } \\
\text { responses, avg } \\
\text { slope of EDA } \\
\text { response, EMG } \\
\text { number of con- } \\
\text { tractions, EMG \% } \\
\text { of activity, mean } \\
\text { temperature, } \\
\text { gradient of linear } \\
\text { regression of } \\
\text { temp }\end{array}$ & & $\begin{array}{l}\text { Physiological } \\
\text { cues (heart } \\
\text { rate variabil- } \\
\text { ity, breath rate, } \\
\text { EDA, EMG, skin } \\
\text { temperature) }\end{array}$ & Physilogical cues & Threshold & $\begin{array}{l}\text { Mood (activated, } \\
\text { relaxed) }\end{array}$ \\
\hline $\begin{array}{l}\text { [Groh et al. } \\
2010]\end{array}$ & $\begin{array}{l}\text { IR cameras, } \\
\text { IR beacons }\end{array}$ & $\begin{array}{l}\text { users spatial } \\
\text { formation } \\
\text { through IR } \\
\text { beacons }\end{array}$ & $\begin{array}{l}\text { IR cameras and } \\
\text { beacons }\end{array}$ & & $\begin{array}{l}\text { interpersonal dis- } \\
\text { tance and spatial } \\
\text { arrangement }\end{array}$ & & & \\
\hline $\begin{array}{l}P B N \text { [Keally } \\
\text { et al. 2011] }\end{array}$ & $\begin{array}{l}\text { accelerometer, } \\
\text { GPS, WiFi, } \\
\text { external(2- } \\
\text { axis ac- } \\
\text { celerome- } \\
\text { ter,microphone, } \\
\text { light, and } \\
\text { tempera- } \\
\text { ture) }\end{array}$ & & $\begin{array}{ll}\text { On-body } & \text { sensor } \\
\text { data } & \end{array}$ & $\begin{array}{l}\text { Pearson corre- } \\
\text { lation coeffi- } \\
\text { cient, Adaboost, } \\
\text { Kullback-Leibler } \\
\text { divergence }\end{array}$ & $\begin{array}{l}\text { Activity classifi- } \\
\text { cation }\end{array}$ & & & \\
\hline
\end{tabular}




\begin{tabular}{|c|c|c|c|c|c|c|c|c|}
\hline \multirow{3}{*}{$\begin{array}{l}\text { System } \\
\text { Meeting Me- } \\
\text { diator [Kim } \\
\text { et al. 2008] }\end{array}$} & \multirow{3}{*}{$\begin{array}{l}\text { Sensing } \\
\text { Microphone, } \\
\text { Bluetooth }\end{array}$} & \multirow{3}{*}{$\begin{array}{l}\text { Social } \\
\text { Interaction } \\
\text { Conversation } \\
\text { detection, } \\
\text { Bluetooth } \\
\text { discovery }\end{array}$} & \multirow{2}{*}{\multicolumn{3}{|c|}{$\begin{array}{l}\text { Behavioural Cues } \\
\text { Method }\end{array}$}} & \multirow{2}{*}{\multicolumn{3}{|c|}{ Social Behaviour }} \\
\hline & & & & & & & & Target \\
\hline & & & $\begin{array}{l}\text { Total speaking } \\
\text { time, Overlap } \\
\text { speaking time, } \\
\text { Turn taking per } \\
\text { sec, avg length } \\
\text { of speech, avg } \\
\text { speaking energy, } \\
\text { avg speaking } \\
\text { speed }\end{array}$ & & $\begin{array}{l}\text { Auditory cues } \\
\text { (conversation, } \\
\text { silence, turn- } \\
\text { taking) }\end{array}$ & $\begin{array}{lr}\text { speaking } & \text { time, } \\
\text { avg } & \text { speech } \\
\text { length, } & \text { vari- } \\
\text { ation } & \text { speech } \\
\text { energy, variation } \\
\text { in movement, } \\
\text { questionnaire } \\
\text { data }\end{array}$ & & Dominance \\
\hline $\begin{array}{l}\text { BeWell [Lane } \\
\text { et al. 2012] }\end{array}$ & $\begin{array}{l}\text { GPS, ac- } \\
\text { celerometer } \\
\text { and micro- } \\
\text { phone }\end{array}$ & & $\begin{array}{l}\text { accelerometer, } \\
\text { GPS, microphone } \\
\text { data, frequency } \\
\text { and duration } \\
\text { phone charging, } \\
\text { time stationary } \\
\text { or silent sound } \\
\text { environment }\end{array}$ & $\begin{array}{l}\text { [Lu et al. } \\
2009][\text { Lu et al. } \\
2010]\end{array}$ & $\begin{array}{l}\text { Conversation, } \\
\text { physical activity }\end{array}$ & $\begin{array}{lr}\text { Sleep, } & \text { physical } \\
\text { activity, } & \text { social } \\
\text { interaction }\end{array}$ & & Well-being \\
\hline $\begin{array}{l}\text { [Lane et al. } \\
\text { 2011] }\end{array}$ & $\begin{array}{l}\text { GPS, ac- } \\
\text { celerometer, } \\
\text { microphone }\end{array}$ & & $\begin{array}{l}\text { accelerometer, } \\
\text { microphone data, } \\
\text { GPS trajectory, } \\
\text { stay points, lo- } \\
\text { cation interest } \\
\text { etc. }\end{array}$ & $\begin{array}{l}\text { [Lu et al. } \\
\text { 2010], naive } \\
\text { Bayes, smoothing } \\
\text { Markov model }\end{array}$ & $\begin{array}{lr}\text { Activity } & \text { de- } \\
\text { tection, } & \text { Social } \\
\text { context } & \end{array}$ & & & \\
\hline $\begin{array}{l}\text { PeopleTones [Li } \\
\text { et al. 2008] }\end{array}$ & $\begin{array}{l}\text { GSM cell } \\
\text { tower }\end{array}$ & $\begin{array}{l}\text { Cell tower } \\
\text { based }\end{array}$ & Cellular data & proximity-ratio & $\begin{array}{l}\text { Interpersonal } \\
\text { distance (Proxim- } \\
\text { ity) }\end{array}$ & & & \\
\hline $\begin{array}{l}\text { PhoneMonitor [L } \\
\text { et al. 2013a] }\end{array}$ & $\begin{array}{l}\text { ilikluetooth, } \\
\text { light sensor, } \\
\text { WiFi, GPS, } \\
\text { cellular data, } \\
\text { device info }\end{array}$ & $\begin{array}{l}\text { Bluetooth } \\
\text { proximity }\end{array}$ & Bluetooth RSSI & $\begin{array}{l}\text { Probabilistic } \\
\text { model }\end{array}$ & $\begin{array}{l}\text { Interpersonal } \\
\text { distance (Proxim- } \\
\text { ity) }\end{array}$ & & & \\
\hline $\begin{array}{l}{\left[\begin{array}{l}\text { Lee } \\
2012]\end{array}\right.} \\
\text { et al. }\end{array}$ & $\begin{array}{l}\text { Camera, ac- } \\
\text { celerometer, } \\
\text { orientation } \\
\text { sensor }\end{array}$ & & $\begin{array}{l}\text { User's image, ac- } \\
\text { celerometer, ori- } \\
\text { entation data }\end{array}$ & $\begin{array}{l}\text { face detection, } \\
\text { threshold based } \\
\text { hand detection, } \\
\text { avg device tilt }\end{array}$ & Head posture & & & \\
\hline $\begin{array}{l}\text { SocioPhone [Lee } \\
\text { et al. } 2013 \mathrm{~b}]\end{array}$ & Microphone & $\begin{array}{l}\text { Conversation } \\
\text { and turn- } \\
\text { taking detec- } \\
\text { tion }\end{array}$ & $\begin{array}{l}300 \text { ms-frames: } \\
\text { power, average } \\
\text { of the square, } \\
\text { decibel given } \\
\text { sound pressure } \\
\text { level, } \\
\text { ZCR, RMS }\end{array}$ & $\begin{array}{l}\text { multi-class SVM, } \\
\text { two consecutive } \\
\text { window frames } \\
\text { to same cluster }\end{array}$ & $\begin{array}{ll}\text { Auditory } & \text { (Con- } \\
\text { versation, } & \text { turn- } \\
\text { taking) } & \end{array}$ & $\begin{array}{l}\text { Conversation, } \\
\text { turn-taking }\end{array}$ & SVM & $\begin{array}{l}\text { Dominance, } \\
\text { sparseness, } \\
\text { skewness, inter- } \\
\text { activity }\end{array}$ \\
\hline $\begin{array}{l}{[\mathrm{Li} \text { et }} \\
2013]\end{array}$ & $\begin{array}{l}\text { Microphone, } \\
\text { GPS, Blue- } \\
\text { tooth, calls, } \\
\text { calendar, } \\
\text { BSN }\end{array}$ & $\begin{array}{l}\text { Bluetooth } \\
\text { distance } \\
\text { estimation }\end{array}$ & $\begin{array}{l}\text { Bluetooth RSSI, } \\
\text { Amplitude mod- } \\
\text { ulation, spectral } \\
\text { profile, harmonic- } \\
\text { ity, accelerometer } \\
\text { variation and } \\
\text { raw data, calen- } \\
\text { dar, location }\end{array}$ & $\begin{array}{l}\text { PLM, tree classi- } \\
\text { fier, threshold, }\end{array}$ & $\begin{array}{l}\text { Intepersonal } \\
\text { distance (prox- } \\
\text { imity), auditory } \\
\text { (conversation), } \\
\text { body posture, } \\
\text { social context }\end{array}$ & & & \\
\hline $\begin{array}{l}\text { Moodscope [LiKa } \\
\text { et al. 2013] }\end{array}$ & $\begin{array}{lc}\text { andWice } & \text { us- } \\
\text { age, } & \text { SMS, } \\
\text { calls, } & \text { loca- } \\
\text { tion } & \end{array}$ & & & & & $\begin{array}{lr}\text { emails, } & \text { SMS, } \\
\text { calls, } & \text { website } \\
\text { domains, } & \text { apps, } \\
\text { locations, } & \text { user } \\
\text { input } & \\
\end{array}$ & $\begin{array}{l}\text { histograms, } \\
\text { multi-linear } \\
\text { regression }\end{array}$ & $\begin{array}{lr}\text { Mood } & \text { (average } \\
73 \%, & \text { generic } \\
66 \%, & \text { personal } \\
93 \%) & \\
\end{array}$ \\
\hline $\begin{array}{l}{\left[\begin{array}{l}\text { Liu et } \\
2012]\end{array}\right.} \\
\end{array}$ & $\begin{array}{l}\text { Orientation } \\
\text { sensor }\end{array}$ & & $\begin{array}{l}\text { Orientation sen- } \\
\text { sor data }\end{array}$ & $\begin{array}{l}\text { Threshold for } \\
\text { different orienta- } \\
\text { tions }\end{array}$ & Body posture & & & \\
\hline $\begin{array}{l}\text { uWave [Liu } \\
\text { et al. 2009] }\end{array}$ & Accelerometer & & $\begin{array}{l}\text { Quantised, dis- } \\
\text { crete time series }\end{array}$ & $\begin{array}{lr}\text { DTW } & \text { match- } \\
\text { ing, minimum } \\
\text { distance, } & \text { with } \\
\text { feedback } & \end{array}$ & $\begin{array}{l}\text { Gesture recogni- } \\
\text { tion }\end{array}$ & & & \\
\hline
\end{tabular}




\begin{tabular}{|c|c|c|c|c|c|c|c|c|}
\hline \multirow{2}{*}{ System } & \multirow{2}{*}{ Sensing } & \multirow{2}{*}{$\begin{array}{l}\text { Social } \\
\text { Interaction }\end{array}$} & \multicolumn{3}{|c|}{ Behavioural Cues } & \multicolumn{3}{|c|}{ Social Behaviour } \\
\hline & & & Features & Method & Target & Features & Method & Target \\
\hline $\begin{array}{l}\text { Guoguo [Liu } \\
\text { et al. 2013b] }\end{array}$ & $\begin{array}{l}\text { Microphone, } \\
\text { speakers }\end{array}$ & & $\begin{array}{l}\text { high-band acous- } \\
\text { tic signals }\end{array}$ & ToA & $\begin{array}{l}\text { Interpersonal } \\
\text { distance (dis- } \\
\text { tance estimation) }\end{array}$ & & & \\
\hline $\begin{array}{l}\text { SpeakerSense [L } \\
\text { et al. 2011] }\end{array}$ & $\begin{array}{l}\text { External and } \\
\text { internal mi- } \\
\text { crophones }\end{array}$ & $\begin{array}{l}\text { Conversation } \\
\text { and speaker } \\
\text { identifica- } \\
\text { tion }\end{array}$ & $\begin{array}{l}\text { ZCR, RMS, Low } \\
\text { energy frame } \\
\text { rate, } \\
\text { pitch }\end{array}$ & $\begin{array}{ll}\text { Decision } & \text { tree, } \\
\text { GMM, EM } & \end{array}$ & $\begin{array}{ll}\text { Audiroty } & \text { (Con- } \\
\text { versation, } & \text { turn- } \\
\text { taking) } & \end{array}$ & & & \\
\hline $\begin{array}{l}\text { StressSense [Lu } \\
\text { et al. 2012] }\end{array}$ & $\begin{array}{l}\text { Microphone } \\
\text { (microcone } \\
\text { indoors) }\end{array}$ & Conversation & $\begin{array}{lr}\text { statistics } & \text { pitch, } \\
\text { spectral ren- } \\
\text { troid, high fre- } \\
\text { quency ratio, } \\
\text { speaking rate, } \\
\text { MFCCs, TEO- } \\
\text { CB-AutoEnv }\end{array}$ & $\begin{array}{l}\text { GMM with diag- } \\
\text { onal covariance } \\
\text { matrix, EM, MAP } \\
\text { (adaptation) }\end{array}$ & $\begin{array}{l}\text { Auditory (Con- } \\
\text { versation) }\end{array}$ & & & Stress \\
\hline $\begin{array}{l}\text { SoundSense [Lu } \\
\text { et al. 2009] }\end{array}$ & Microphone & & $\begin{array}{l}\text { ZCR, Low en- } \\
\text { ergy frame rate, } \\
\text { spectral flux, } \\
\text { spectral rolloff, } \\
\text { spectral centroid, } \\
\text { bandwidth, nor- } \\
\text { malised weighted } \\
\text { phase deviation, } \\
\text { relative spectral } \\
\text { entropy, MFCC }\end{array}$ & $\begin{array}{lr}\text { Decision } & \text { tree, } \\
\text { Markov mod- } \\
\text { els, smoothing, } \\
\text { Bayes, HMM } \\
\text { smoothing }\end{array}$ & $\begin{array}{l}\text { Auditory (Con- } \\
\text { versation), Social } \\
\text { Context }\end{array}$ & & & \\
\hline $\begin{array}{l}\text { VibeFones [Mada } \\
\text { and Pent- } \\
\text { land 2006] }\end{array}$ & $\begin{array}{l}\text { aMicrophone, } \\
\text { Bluetooth, } \\
\text { SMS }\end{array}$ & $\begin{array}{l}\text { Bluetooth } \\
\text { discovery }\end{array}$ & $\begin{array}{l}\text { Microphone } \\
\text { data [Pentland } \\
\text { 2007] }\end{array}$ & $\begin{array}{l}\text { multi-layer } \\
\text { HMM, Markov } \\
\text { process }\end{array}$ & $\begin{array}{l}\text { Auditory (Con- } \\
\text { versation, turn- } \\
\text { taking, utter- } \\
\text { ances, influence), } \\
\text { Physical activity }\end{array}$ & $\begin{array}{lc}\text { Auditory } & \text { (Con- } \\
\text { versation, } & \text { turn- } \\
\text { taking, } & \text { utter- } \\
\text { ances, influence) }\end{array}$ & $\begin{array}{l}\text { z-scored percent- } \\
\text { age of speaking } \\
\text { time, z-scored } \\
\text { influence on } \\
\text { turn-taking } \\
\text { (HMM), z-score } \\
\text { of pitch and am- } \\
\text { plitude variation, } \\
\text { z-score of short } \\
\text { utterances }\end{array}$ & $\begin{array}{l}\text { Stress, activity } \\
\text { level, engage- } \\
\text { ment, emphasis, } \\
\text { mirroring }\end{array}$ \\
\hline $\begin{array}{l}\text { [Matic et al. } \\
\text { 2012] }\end{array}$ & $\begin{array}{l}\text { WiFi, Mag- } \\
\text { netometer, } \\
\text { external ac- } \\
\text { celerometer }\end{array}$ & $\begin{array}{l}\text { Interpersonal } \\
\text { distance, } \\
\text { relative ori- } \\
\text { entation, } \\
\text { conversation }\end{array}$ & $\begin{array}{l}\text { 10-sample win- } \\
\text { dow WiFi RSSI, } \\
\text { device azimuth, } \\
\text { power spectral } \\
\text { density of mean, } \\
\text { maximal, mini- } \\
\text { mal, and integral } \\
\text { of } 10 \text { sec audio }\end{array}$ & $\begin{array}{l}\text { Naïve Bayes } \\
\text { with KDE, raw } \\
\text { azimuth, noise } \\
\text { cancellation, } \\
\text { Naïve Bayes with } \\
\text { KDE }\end{array}$ & $\begin{array}{l}\text { Interpersonal } \\
\text { distance (Prox- } \\
\text { imity), Relative } \\
\text { spatial arrange- } \\
\text { ment, Auditory } \\
\text { (conversation) }\end{array}$ & & & \\
\hline $\begin{array}{l}\text { [Muaremi } \\
\text { et al. 2013] }\end{array}$ & $\begin{array}{l}\text { Microphone, } \\
\text { Accelerom- } \\
\text { eter, GPS, } \\
\text { phone calls, } \\
\text { address } \\
\text { book, calen- } \\
\text { dar, battery, } \\
\text { Biomonitor- } \\
\text { ing sensor }\end{array}$ & phone calls & $\begin{array}{l}\text { mean accelera- } \\
\text { tion variation, } \\
\text { sleep duration, } \\
\text { Mean RR, std RR, } \\
\text { RMS RR, } 50 \mathrm{~ms} \\
\text { difference RR } \\
\text { intervals, HRV } \\
\text { index, triangular } \\
\text { interpolation, } \\
\text { approximate en- } \\
\text { tropy, coefficients } \\
\text { of Poincoir, LF, } \\
\text { HF, LF/HF }\end{array}$ & & $\begin{array}{l}\text { Physical activity, } \\
\text { Physiological } \\
\text { (heart rate) }\end{array}$ & $\begin{array}{l}\text { Physical activity, } \\
\text { physiological } \\
\text { cues }\end{array}$ & binomial logit & Stress \\
\hline $\begin{array}{l}\text { BeepBeep [Peng } \\
\text { et al. 2007] }\end{array}$ & $\begin{array}{l}\text { Microphone, } \\
\text { spearker }\end{array}$ & & $\begin{array}{l}\text { peaks, sharpness } \\
\text { of a peak, maxi- } \\
\text { mum peak }\end{array}$ & $\begin{array}{l}\text { ToA combined } \\
\text { with threshold }\end{array}$ & $\begin{array}{l}\text { Interpersonal } \\
\text { distance }\end{array}$ & & & \\
\hline $\begin{array}{l}\text { [Peternel } \\
\text { et al. 2012] }\end{array}$ & $\begin{array}{l}\text { Accelerometer, } \\
\text { calls, ringer, } \\
\text { GPS, micro- } \\
\text { phone }\end{array}$ & & $\begin{array}{l}\text { call status, ringer } \\
\text { status, ambient } \\
\text { sound, location, } \\
\text { accelerometer } \\
\text { data }\end{array}$ & $\begin{array}{l}\text { threshold based } \\
\text { categorisation }\end{array}$ & $\begin{array}{l}\text { Social context, } \\
\text { Physical activity }\end{array}$ & $\begin{array}{l}\text { Social context, } \\
\text { Physical activity }\end{array}$ & HMM, Viterbi & Stress \\
\hline
\end{tabular}

\section{https://mc.manuscriptcentral.com/csur}




\begin{tabular}{|c|c|c|c|c|c|c|c|c|}
\hline \multirow{2}{*}{ System } & \multirow{2}{*}{ Sensing } & \multirow{2}{*}{$\begin{array}{l}\text { Social } \\
\text { Interaction }\end{array}$} & \multicolumn{3}{|c|}{ Behavioural Cues } & \multicolumn{3}{|c|}{ Social Behaviour } \\
\hline & & & Features & Method & Target & Features & Method & Target \\
\hline $\begin{array}{l}\text { [Pino and } \\
\text { Kavasidis } \\
2012]\end{array}$ & $\begin{array}{l}\text { Accelerometer, } \\
\text { camera }\end{array}$ & & $\begin{array}{l}\text { Haar-like fea- } \\
\text { tures, rectangles, } \\
\text { sum of pixel inte- } \\
\text { sities, difference } \\
\text { among adjacent } \\
\text { rectangles }\end{array}$ & $\begin{array}{l}\text { CAMSHIFT, } \\
\text { Starburst algo- } \\
\text { rithm, Haar Eye } \\
\text { Detection }\end{array}$ & $\begin{array}{l}\text { Facial } \\
\text { tracking) }\end{array}$ & & & \\
\hline $\begin{array}{l}\text { [Razak and } \\
\text { Abidin 2005] }\end{array}$ & Microphone & & $\begin{array}{l}\text { Energy, pitch, } \\
\text { LPC coefficients, } \\
\text { duration, pitch, } \\
\text { jitter }\end{array}$ & & Auditory (speech) & Auditory & 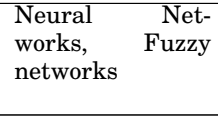 & $\begin{array}{l}\text { Emotion (62.35\%, } \\
\text { accuracy) }\end{array}$ \\
\hline $\begin{array}{l}\text { CONSORTS- } \\
S \quad \text { [Sashima } \\
\text { et al. 2008] }\end{array}$ & $\begin{array}{l}\text { ECG, ac- } \\
\text { celerometer, } \\
\text { thermome- } \\
\text { ter, hy- } \\
\text { grometers, } \\
\text { microphone } \\
\text { array }\end{array}$ & & $\begin{array}{l}\text { latest, maxi- } \\
\text { mum, minimum, } \\
\text { average }\end{array}$ & $\begin{array}{l}\text { DFT, SVM, Near- } \\
\text { est Neighbour }\end{array}$ & $\begin{array}{l}\text { Physiological } \\
\text { (heart rate, skin } \\
\text { temperature), } \\
\text { Posture, Physical } \\
\text { activity }\end{array}$ & & & \\
\hline $\begin{array}{l}\text { [Seiter et al. } \\
\text { 2013] }\end{array}$ & $\begin{array}{l}\text { Accelerometer, } \\
\text { GPS, barom- } \\
\text { eter }\end{array}$ & & $\begin{array}{l}\text { location clusters, } \\
\text { transition clus- } \\
\text { ters, rectified } \\
\text { signal, intensity, } \\
\text { cadence, number } \\
\text { of steps, number } \\
\text { of instance in } \\
\text { climbing stairs }\end{array}$ & t-tset & $\begin{array}{l}\text { Physical activity, } \\
\text { Social Context }\end{array}$ & & & \\
\hline $\begin{array}{l}\text { [Singh et al. } \\
\text { 2013a] }\end{array}$ & $\begin{array}{l}\text { Bluetooth, } \\
\text { calls, SMS, } \\
\text { question- } \\
\text { naire }\end{array}$ & $\begin{array}{l}\text { Bluetooth } \\
\text { discovery }\end{array}$ & & & & $\begin{array}{l}\text { interaction } \\
\text { events, differ- } \\
\text { ent contacts, } \\
\text { interaction diver- } \\
\text { sity, number of } \\
\text { total purchases, } \\
\text { colocation }\end{array}$ & Naïve Bayes & $\begin{array}{lr}\text { Diversity } & (69 \% \\
\text { accuracy), } & \text { Loy- } \\
\text { alty (69\% } & \text { ac- } \\
\text { curacy), } & \text { Over- } \\
\text { spending } & (77 \% \\
\text { accuracy) } & \end{array}$ \\
\hline $\begin{array}{l}\text { [Srinivasan } \\
\text { and Phan } \\
\text { 2012] }\end{array}$ & Accelerometer & & $\begin{array}{l}\text { the highest mag- } \\
\text { nitude frequency, } \\
\text { magnitude of } \\
\text { highest magni- } \\
\text { tude frequency, } \\
\text { weighted mean } \\
\text { of top-5 high- } \\
\text { est magnitude } \\
\text { frequencies, } \\
\text { weighted vari- } \\
\text { ance of the top-5 } \\
\text { highest magni- } \\
\text { tude frequencies }\end{array}$ & $\begin{array}{l}\text { C4.5 decision } \\
\text { tree, with con- } \\
\text { fidence thresh- } \\
\text { old, with pre- } \\
\text { classification }\end{array}$ & $\begin{array}{l}\text { Activity recogni- } \\
\text { tion }\end{array}$ & & & \\
\hline $\begin{array}{l}\text { [Staiano } \\
\text { et al. 2012] }\end{array}$ & $\begin{array}{l}\text { Bluetooth, } \\
\text { calls, survey }\end{array}$ & $\begin{array}{l}\text { Bluetooth } \\
\text { discovery }\end{array}$ & & & & $\begin{array}{lr}\text { centrality, } & \text { ef- } \\
\text { ficiency, } & \text { tran- } \\
\text { sitivity, } & \text { triadic } \\
\text { measures } & \end{array}$ & Random forests & $\begin{array}{l}\text { Personality } \\
\text { traits (agree- } \\
\text { ableness, con- } \\
\text { scientiousness, } \\
\text { extroversion, } \\
\text { neuroticism, } \\
\text { openness) }\end{array}$ \\
\hline $\begin{array}{l}\text { [Stehl et al. } \\
2011]\end{array}$ & RFID & $\begin{array}{l}\text { Interpersonal } \\
\text { distance } \\
\text { with par- } \\
\text { tial relative } \\
\text { orientation }\end{array}$ & $\mathrm{RF}$ signal & $\begin{array}{l}\text { Signal strength } \\
\text { interpretation }\end{array}$ & $\begin{array}{l}\text { Interpersonal } \\
\text { distance, partial } \\
\text { relative spatial } \\
\text { arrangement }\end{array}$ & & & \\
\hline
\end{tabular}




\begin{tabular}{|c|c|c|c|c|c|c|c|c|}
\hline \multirow{2}{*}{ System } & \multirow{2}{*}{ Sensing } & \multirow{2}{*}{$\begin{array}{l}\text { Social } \\
\text { Interaction }\end{array}$} & \multicolumn{3}{|c|}{ Behavioural Cues } & \multicolumn{3}{|c|}{ Social Behaviour } \\
\hline & & & Features & Method & Target & Features & Method & Target \\
\hline $\begin{array}{l}{[\text { Sun et al. }} \\
2012]\end{array}$ & $\begin{array}{l}\text { Accelerometer, } \\
\text { ECG, GSR }\end{array}$ & & $\begin{array}{l}\text { Mean RR, Std } \\
\text { RR, Mean HR, } \\
\text { Std HR RMSSD, } \\
\text { pNN50, LF, HF, } \\
\text { LF/HF ratio, } \\
\text { Mean SCL, Std } \\
\text { SCL, Total mag- } \\
\text { nitude, Duration, } \\
\text { and Number of } \\
\text { startle responses, } \\
\text { Mean, std, en- } \\
\text { ergy of XYZ axis, } \\
\text { Correlation coef- } \\
\text { ficient of XY, YZ, } \\
\text { and ZX }\end{array}$ & $\begin{array}{l}\text { SVM, Bayesian } \\
\text { network, decision } \\
\text { tree }\end{array}$ & $\begin{array}{l}\text { Physiological, Ac- } \\
\text { tivity }\end{array}$ & $\begin{array}{l}\text { Physiological, ac- } \\
\text { tivity }\end{array}$ & SVM & Stress \\
\hline $\begin{array}{l}\text { eMoto [Sund- } \\
\text { ström et al. } \\
\text { 2005] }\end{array}$ & $\begin{array}{l}\text { external sty- } \\
\text { lus }\end{array}$ & & $\begin{array}{l}\text { pressure, shak- } \\
\text { ing of stylus }\end{array}$ & Laban-notation & $\begin{array}{l}\text { Physical activity, } \\
\text { Gestures }\end{array}$ & Activity, gestures & & $\begin{array}{l}\text { Emotion } \\
\text { (arousal, valence) }\end{array}$ \\
\hline $\begin{array}{l}\text { TagMobile [Tag } \\
\text { Mobile 2003] }\end{array}$ & RFID & $\begin{array}{l}\text { Interpersonal } \\
\text { distance and } \\
\text { spatial ar- } \\
\text { rangement }\end{array}$ & $\begin{array}{ll}\mathrm{RF} & \text { signal } \\
\text { strength }\end{array}$ & Triangulation & $\begin{array}{l}\text { Interpersonal } \\
\text { distance and spa- } \\
\text { tial arrangement }\end{array}$ & & & \\
\hline $\begin{array}{l}\text { UbiSense [Ubist } \\
\text { 2003] }\end{array}$ & $\begin{array}{l}\text { nsidtra- } \\
\text { wideband }\end{array}$ & $\begin{array}{l}\text { Interpersonal } \\
\text { distance and } \\
\text { spatial ar- } \\
\text { rangement }\end{array}$ & $\begin{array}{l}\text { Ultra-wideband } \\
\text { signal strength }\end{array}$ & TDoA, ToA & $\begin{array}{l}\text { Interpersonal } \\
\text { distance and spa- } \\
\text { tial arrangement }\end{array}$ & & & \\
\hline $\begin{array}{l}R F- \\
\text { Beep [Uddin } \\
\text { and Nadeem } \\
\text { 2013] }\end{array}$ & $\begin{array}{l}\text { Microphone, } \\
\text { speakers, } \\
\text { WiFi }\end{array}$ & & $\begin{array}{l}\text { WiFi beacon } \\
\text { frame, acoustic } \\
\text { beacon signal }\end{array}$ & TDoA & $\begin{array}{l}\text { Interpersonal } \\
\text { distance }\end{array}$ & & & \\
\hline $\begin{array}{l}\text { [Vertegaal } \\
\text { et al. 2002] }\end{array}$ & $\begin{array}{l}\text { EyeContact } \\
\text { sensor, mi- } \\
\text { crophone }\end{array}$ & $\begin{array}{l}\text { Eye contact } \\
\text { and speech } \\
\text { detection }\end{array}$ & $\begin{array}{l}\text { pupil detected } \\
\text { from } \\
\text { energy of ther, } \\
\text { voice }\end{array}$ & & $\begin{array}{l}\text { Auditory (conver- } \\
\text { sation, silence), } \\
\text { Facial } \\
\text { tracking) }\end{array}$ & & & \\
\hline $\begin{array}{l}\text { [Vinciarelli } \\
\text { et al. 2012b] }\end{array}$ & Microphone & & $\begin{array}{l}\text { syllables, har- } \\
\text { monicity, spectral } \\
\text { centroid, skew- } \\
\text { ness, kurtosis, } \\
\text { jitter, shimmer } \\
\text { etc. and statistics }\end{array}$ & threshold & Auditory & $\begin{array}{l}\text { Auditory (turn- } \\
\text { taking, silences, } \\
\text { laughter, utter- } \\
\text { ances) }\end{array}$ & & $\begin{array}{l}\text { Personality traits } \\
\text { (agreeableness, } \\
\text { conscientious- } \\
\text { ness, extrover- } \\
\text { sion, neuroti- } \\
\text { cism, openness), } \\
\text { Sociability }\end{array}$ \\
\hline $\begin{array}{l}\text { [Wang et al. } \\
\text { 2009] }\end{array}$ & $\begin{array}{l}\text { GPS, ac- } \\
\text { celerometer, } \\
\text { microphone, } \\
\text { WiFi }\end{array}$ & & $\begin{array}{ll}\begin{array}{l}\text { energy, } \\
\text { peaks }\end{array} & \text { FFT, }\end{array}$ & $\begin{array}{l}\text { subband } \\
\text { centroid } \begin{array}{r}\text { his- } \\
\text { togram, thresh- } \\
\text { old }\end{array}\end{array}$ & $\begin{array}{l}\text { Social context, } \\
\text { activity }\end{array}$ & & & \\
\hline $\begin{array}{l}\text { [Wang et al. } \\
\text { 2013] }\end{array}$ & Bluetooth & & Bluetooth RSSI & triangulation & $\begin{array}{l}\text { Interpersonal } \\
\text { distance, spatial } \\
\text { arrangement }\end{array}$ & & & \\
\hline $\begin{array}{l}{\left[\begin{array}{l}\text { [Wu } \\
\text { 2009] }\end{array}\right.} \\
\text { al }\end{array}$ & Accelerometer & & $\begin{array}{l}\text { mean, energy, en- } \\
\text { tropy, std of am- } \\
\text { plitude and corre- } \\
\text { lation }\end{array}$ & $\begin{array}{l}\text { Frame-based } \\
\text { Descriptor multi- } \\
\text { class SVM }\end{array}$ & Gesture & & & \\
\hline $\begin{array}{l}\text { TWyatt et al. } \\
2011]\end{array}$ & & & $\begin{array}{l}\text { (relative) spec- } \\
\text { tral entropy, } \\
\text { (maximum) auto- } \\
\text { correlation peaks } \\
\text { log and value, } \\
\text { energy }\end{array}$ & HMM, EM, MAP & $\begin{array}{l}\text { Auditory (con- } \\
\text { versation, pitch, } \\
\text { rate, turn-taking) } \\
\text {, Social context }\end{array}$ & & & \\
\hline $\begin{array}{l}\text { Whistle [Xu } \\
\text { et al. 2011] }\end{array}$ & $\begin{array}{l}\text { Microphone, } \\
\text { speaker }\end{array}$ & & $\begin{array}{l}\text { auto-correlation, } \\
\text { peaks, cross- } \\
\text { correlation, } \\
\text { maximum peaks }\end{array}$ & $\begin{array}{l}\text { TD2S, TDoA, tri- } \\
\text { angulation }\end{array}$ & $\begin{array}{l}\text { Interpersonal } \\
\text { distance, spatial } \\
\text { arrangement }\end{array}$ & & & \\
\hline
\end{tabular}




\begin{tabular}{|c|c|c|c|c|c|c|c|c|}
\hline \multirow{2}{*}{ System } & \multirow[t]{2}{*}{ Sensing } & \multirow{2}{*}{$\begin{array}{l}\text { Social } \\
\text { Interaction }\end{array}$} & \multicolumn{3}{|c|}{ Behavioural Cues } & \multicolumn{3}{|c|}{ Social Behaviour } \\
\hline & & & Features & Method & Target & Features & Method & Target \\
\hline $\begin{array}{l}\text { [Yan et al. } \\
2012]\end{array}$ & Accelerometer & & $\begin{array}{l}\text { mean, variance, } \\
\text { magnitude, co- } \\
\text { variance, energy, } \\
\text { entropy, FFT } \\
\text { coefficients }\end{array}$ & $\begin{array}{l}\text { J48 adaptive de- } \\
\text { cision tree }\end{array}$ & Activity & & & \\
\hline [Yang 2009] & Accelerometer & & $\begin{array}{l}\text { Moving aver- } \\
\text { aged window, } \\
\text { mean, std, ZCR, } \\
75 \% \text { percentile, } \\
\text { interquartile } \\
\text { range, power } \\
\text { spectrum cen- } \\
\text { troid, entropy } \\
\text { of the vertical } \\
\text { and horizontal } \\
\text { components, } \\
\text { cross-correlation, } \\
\text { amplitude of the } \\
\text { vertical compo- } \\
\text { nents, magnitude } \\
\text { of the horizontal } \\
\text { components }\end{array}$ & $\begin{array}{l}\text { decision tree, } \\
\text { k-means cluster- } \\
\text { ing, HMM-based } \\
\text { Viterbi algorithm } \\
\text { smoothing }\end{array}$ & Activity & & & \\
\hline $\begin{array}{l}\text { Visage [Yang } \\
\text { et al. 2013] }\end{array}$ & $\begin{array}{l}\text { Camera, } \\
\text { motion (ac- } \\
\text { celerometer, } \\
\text { orientation, } \\
\text { gyroscope) }\end{array}$ & & $\begin{array}{l}\text { moving windon } \\
\text { on image, gravity } \\
\text { direction, mo- } \\
\text { tion intensity, } \\
\text { mean-variance } \\
\text { on direction, eye } \\
\text { corners, edges of } \\
\text { mouths }\end{array}$ & $\begin{array}{l}\text { Face detec- } \\
\text { tion (adaboost), } \\
\text { Lucas-Kanade, } \\
\text { CAMSHIFT, de- } \\
\text { vice posture, Pose } \\
\text { from Orthogra- } \\
\text { phy and Scaling } \\
\text { with ITerations } \\
\text { (POSIT), Ac- } \\
\text { tive Appearance } \\
\text { Models }\end{array}$ & $\begin{array}{l}\text { Facial expres- } \\
\text { sion, Head pose }\end{array}$ & $\begin{array}{l}\text { Facial expres- } \\
\text { sions }\end{array}$ & $\begin{array}{l}\text { Fisher Linear } \\
\text { Discriminant } \\
\text { Analysis (Fisher- } \\
\text { face), SVM with } \\
\text { RBF }\end{array}$ & $\begin{array}{l}\text { Anger, disgust, } \\
\text { fear, happiness, } \\
\text { neutral, sad, } \\
\text { surprise }\end{array}$ \\
\hline
\end{tabular}

